\title{
Laboratory Services Series-The Utilization of Scientific Glassblowing in a National Research and Development Laboratory
}

R. M. Farnham

R. W. Poole

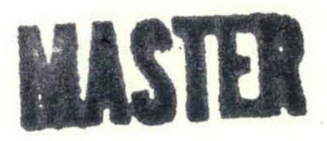




\section{DISCLAIMER}

This report was prepared as an account of work sponsored by an agency of the United States Government. Neither the United States Government nor any agency Thereof, nor any of their employees, makes any warranty, express or implied, or assumes any legal liability or responsibility for the accuracy, completeness, or usefulness of any information, apparatus, product, or process disclosed, or represents that its use would not infringe privately owned rights. Reference herein to any specific commercial product, process, or service by trade name, trademark, manufacturer, or otherwise does not necessarily constitute or imply its endorsement, recommendation, or favoring by the United States Government or any agency thereof. The views and opinions of authors expressed herein do not necessarily state or reflect those of the United States Government or any agency thereof. 


\section{DISCLAIMER}

Portions of this document may be illegible in electronic image products. Images are produced from the best available original document. 


\section{Printed in the United States of America. Available from National Technical Information Service \\ U.S. Department of Commerce 5285 Port Royal Road, Springfield, Virginia 22161 \\ Price: Printed Copy $\$ 4.50$; Microfiche $\$ 2.25$}

This report was prepared as an account of work sponsored by the United States Government. Neither the United States nor the Energy Research and Development Administration/United States Nuclear Regulatory Commission, nor any of their employees, nor any of their contractors, subcontractors, or their employees, makes any warranty, express or implied, or assumes any legal liability or responsibility for the accuracy, completeness or usefulness of any information, apparatus, product or process disclosed, or represents that its use would not infringe privately owned rights. 
1.:- ? $?$

ORNL/ TM -5360

Contract No. W-7405-eng-26

PLANT AND EQUIPMENT DIVISION

LABORATORY SERVICES SERIES - THE UTILIZATION OF SCIENTIFIC GLASSBLOWING IN A NATIONAL. RESEARCH AND DEVELOPMENT LABORATORY

R. M. Farnham

Fabrication Department Superintendent

and

R. W. Poole

Chief Glassblower

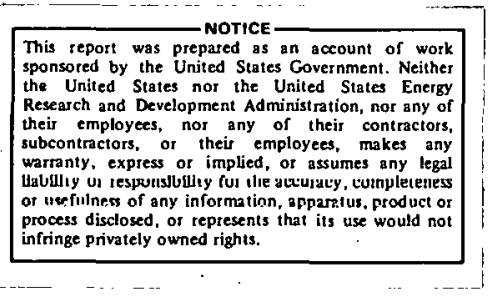

\begin{abstract}
APRIL 1976
\end{abstract}
OAK RIDGE NATIONAL LABORATORY

Oak Ridge, Tennessee $\mathbf{3 7 8 3 0}$ operated by

UNION CARBIDE CORPORATION

for the

ENERGY RESEARCH AND DEVELOPMENT ADMINISTRATION 
THIS PAGE

\section{WAS INTENTIONALLY LEFT BLANK}


CONTENTS

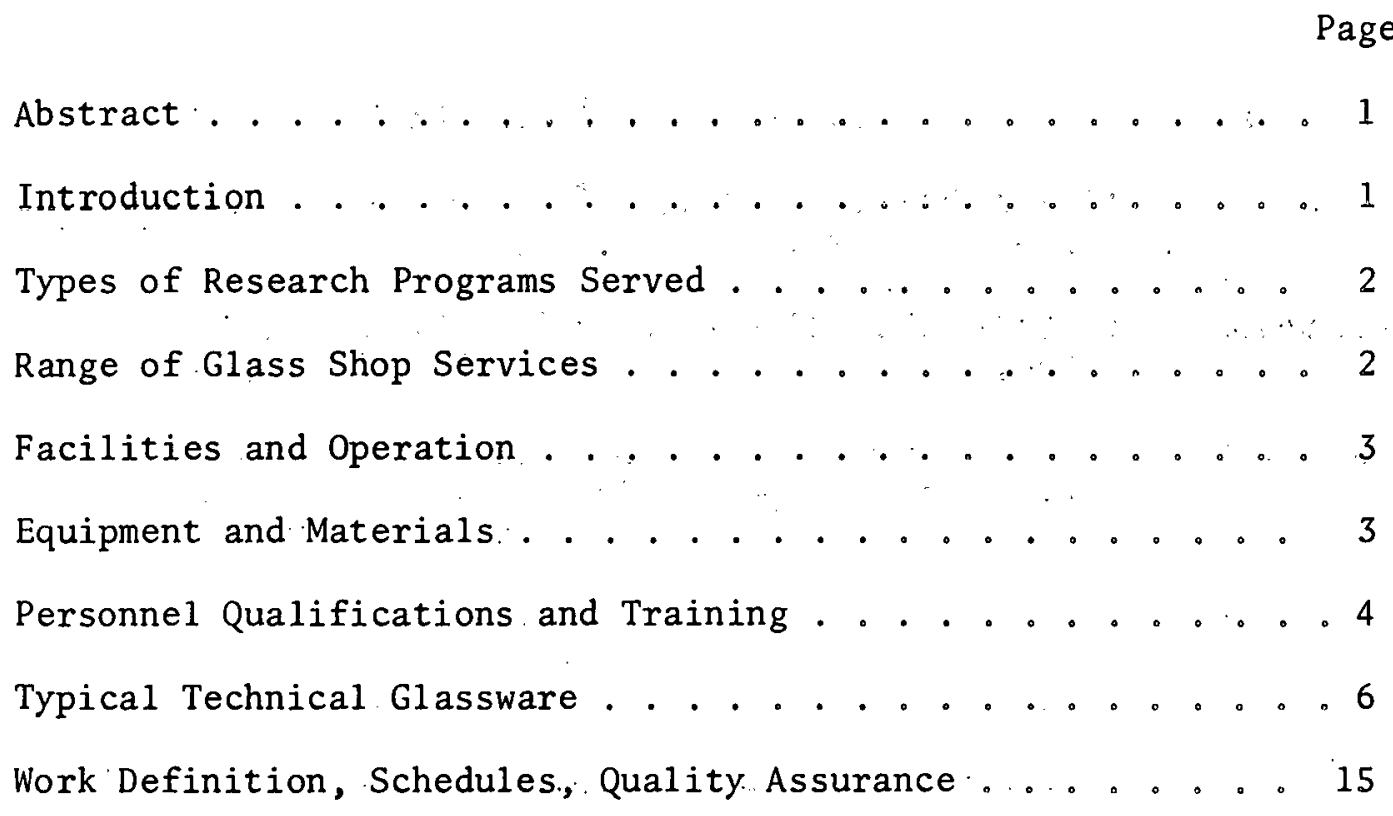




\section{ATTACHMENTS}

Attachment A. - Glass Shop Layout and Equipment List . . . . 17

Attachment B - Stores Catalog Listings of Glassware and Glass Stock. . . . . . . . . . . 18

Attachment C - Criteria Checklist - Scientific Glassblowing Facility . . . . . . . . . . . . . 58

Attachment D. - Organization Chart - Fabrication Department Plant and Equipment Division . . . . . . 60 
LABORATORY SERVICES SERIES . - THE . UTILIZATION.OF..SC.IENT.IFIC GLASSBLOW ING IN A NATIONAL. RESEARCH AND. DEVELOPMENT LABORATORY

\author{
R........ Farnham \\ Fabrication Department Superintendent \\ and \\ R.W. Poole \\ Chief. Glassblower
}

\begin{abstract}
Glassblowing services at a national research and development laboratory provide unique equipment tailored for specific research efforts, small-scale process items for flowsheet demonstrations, and solutions for unusual technical problems such as glass-ceramic unions... Facilities, equipment, and personnel necessary for such services are described.
\end{abstract}

\title{
INTRODUCT ION
}

Since the days of Egyptian and Phoenician dominance, glass was associated with developing civilization. The beginnings of modern physical sciences found glass in the scrvice of the alchemists and other pioneers. Unique properties combination of transparency, strength, formability, cleanability, chemical inertness, and electrical resistance made glass a part of each developing. science such as chemistry, physics, biology, and medicine. Further development nf many specialized glasses...has increased the advantageous applications in science laboratories, resulting in continued broad, use in today's modern research and development.

At Clinton. Laboratory. (to become Oak Ridge National Laboratory), a glassblowing shop was established during World War. II to supply the specific needs of the chemists, physicists, and associated technical people for research and development work in the nuclear-related fields. The original glassblowers were military personnel of the Manhattan Project, some of whom stayed on following discharge from the army. This scientific glassblowing service continues in 1976. 
TYPES. OF . RESEARCH . PROGRAMS . SERVED

The Laboratory has had very. few research programs. since. 1943 which did not require direct.or.indirect glassblowing service. support. A combination. of useful properties continues to make glass. the economic and effective material choice for many scientific laboratory research applications. The large majority of broadly varied. research and development programs throughout ORNL history have utilized the scientific glassblowing services with direct benefit, that may have been anything from bench rack concept. systems to pilot plant process equipment, to analytical and.control applications.

Quartz fiber.electroscopes provided the most accurate early.measurement of ionizing radiation....Glass Geiger tubes.. followed. Quartz fiber balances were:long. among the most sensitive weighing devices. Radioisotope separations chemistry..was. originated in glass. systems. High purity single crystal growing. required glass and quartz envelopes. Low temperature. investigations required effective dewar. vessels of unusual size and shapes.. Separation, handling, and use of radioactive or rare gases.were most often accomplished in glass ampoules and equipment. Special.electron tubes were housed in glass envelopes. Largebore, precision-tapered.glass tube...was used in ion separation columns. Biological.1aboratories. depended..heavily on glass equipment.

\section{RANGE. OF. GLASS. SHOP SERVICES}

The range of glass shop services includes cutting,-grinding, 1apping, forming and molding, chemical silvering, annealing,:-encapsulation, evacuation, fabrication of. special glass equipment. and bench systems, literature search, design or design coordination, preparation of models, and the development of new seals or techniques and seals. to. other materials. The glassblowers repair. or revise a laboratory glass system on location if necessary. .. The chief glassblower often coordinates the glass-associated requirements for machined or fabricated metal parts, for optical finishing, or for optical film coating. 


\section{FACILITIES AND OPERATION}

Scientific: support: work is done in three separate glass. shops as well as in the field. The main shop in Building 450.0NM has been reduced to only two glassblowers and the chief glassblower at this time. ORNL services the $Y-12$. Plant glassblowing requirements from a two-man shop there: in Building 9.766. Also.1ocated in Y-12., a one-man shop serves the ORNL Biology. Division in Building 92.07... Each of. three shops is equipped for the lathe and bench work in glass generally required by the customers served:in. each of these areas..... The glassware requirements differ greatly: among the three locations in the two plants. Biology Division customers use small or miniature items more often than customers in the other two areas. . $\dot{Y}-12$ area customers tend. to require more massive and repetitive fabrications. The. ORNL-situated customers make the broadest variety of lemands for glassblowing and glass-related services. With such differences in mind the chief. glassblower occasionally reassigns glassblowers within the system, matching the peculiar needs of the customers. served and the abilities.of: the. glassblower being assigned. This gives:further: training to the glassblower: and: builds a broadly experienced group able to step into. any of the shops as needed and do: a creditable job. This integrated three-shop. system also lends itself well to the shifting of work or men from one. shop to another, allowing peak-load leveling and assuring.optimum scheduling with efficient utilization of manpower:

\section{EQUIPMENT AND MATERIALS}

Specific glassworking_equipment in each shop includes:

Lathe, Glass

Saw, Glass

Edging Machine

Lap

Dril1

Oven, Annealing.

Vacuum System

$\begin{array}{ccc}\frac{4500 N M}{4} & \frac{9207}{1} & \frac{9766}{3} \\ 2 & 3 & 1 \\ 1 & 1 & 1 \\ 1 & 1 & 1 \\ 2 & 2 & 1 \\ 3 & 2 & 1 \\ 1 & & \end{array}$

The arrangement of equipment and a detailed list of. equipment for the Building 4500NM shop is attached: (Attachment $\mathrm{A}_{0}$ ) 
A11 locations have. well-equipped glassblawing. benches; a good variety of burners, and many hand tools for forming glass.. Adequate exhaust hoods over :each. work station dispose of excess heat: to maintain reasonable working conditions.

The variety and sizes of glassware and stocks.. presently. kept in stores by the Materials Departments are listed on the catalog pages of Attachment B. These materials are the starting point for the glassblowers who modify and combine them in the necessary ways to produce the research equipment: required.: Pyrex and quartz: are the most frequently used materials... Glass tube stocks are seldom precisely straight, round, or of constant diameter over their length. The glassblower must often correct a. tube before it can be incorporated into the job being done.

One note of interest is that the scientific glassware business has remained metric throughout its history.

\section{PERSONNEL QUALIFICATIONS AND TRAINING}

There are. many "types" of glassblowing as.there are glassblowers and employers. Most.glassblowers develop.skill and techniquc according to the training and.the available variety of work. Different employers require that thelr. glassblowers use ceitain techniquos poculiarly necessary for their products. ORNL also has its pecullar reyuifements, and experience has proved it usually best to train glassblowers internally rather than to hire and retrain.

To start a new glass shop for scientific glassblowing one has no choice other than to recruit a competent, experienced glassblower. The prime sources of candidates would include universities, scientific research laboratories, large chemical industries, and laboratory glassware manufacturers. If more than a one-man shop is foreseen, the applicant first hired should. have potential in supervision as a chief glassblower. Additional glassblowers can be less experienced.

For glassblower trainees minimum qualifications include high school education with science courses preferred; general good health and vision; and no respiratory problems or unusual sensitivity to heat. 
The glassblower trainee is instructed progressively in basic tasks with supplemental course work in mathematics. and physics as necessary. Much of the glassware produced.. in task training.is.used. Instruction is geared to the individual and his natural rate of progress. As basic manipulatory. skills are mastered, special. techniques are learned and applied to. different. and increasingly more difficult assignments. As he moves on through the classification ladder to. Junior Glassblower and Assistant Glassblower: grades, the trainee remains under the guidance of one or more Senior Glassblower. When he becomes classified as Glassblower. he will be competent to handle almost any:job routinely submitted to the glass. shop and without additional instruction. After more years of experience, ...the properly prepared person. will. advance to the classification of Senior. Glassublower - able to-plan, design, coordinate, and execute in glass any reasonable requirement. The progression from greenhorn to Senior Glassblower may take from 10. to 15 years.

A senior glassblower at ORNL must have considerable knowledge and skill in scientific. glassblowing as well..as. a. general knowledge of manufacturing processes. and techniques. . He must. have ability in designing, developing, producing, and repairing the most complicated forms of glassware required by: scientific and technical personnel; must know the chemical, . physical,... and working. qualities. of many different glasses; must know and be able to.utilize various. glassblowing and machining tools such as cut -off saws; hot wire, lathes; drills, grinding plates, and other shop devices; must know how to remove,.. repair, and reinstall broken or damaged glass...apparatus; how to anneal. by flame and other methods; how to effect.glass-to-metal seals, ceramic-to-glass seals and graded seals to match expansion. coefficients of other materials; how to mold and press. vitrous articles; press. and slip cast fritted glasses; how to temper heavy glass fittings, : and instruct others. He requires fundamental knowledge of vacuum technique and of other materials for application in glass work. He must be able..to. interpret and to make sketches and drawings in order to exchange information. with requesters, supervisors, and craftsmen. Basic knowledge in physics and chemistry is necessary for discussion and understanding of job.requirements with requesters. He must be able to calculate 
stresses, pressures, strengths, rate of. chemical attack, and other job factors. He must know existing. Laboratory policies and procedures, safety and radiation hazards, pertinent new developments; human relations, services and equipment available in the Department shops, and how to attack unique problems.

\section{TYPICAL. TECHNICAL. GLASSWARE}

The following photographs (Figs. 1-9). of actual.. research glassware used in past programs at Oak. Ridge National. Laboratory constitute a very limited. indication of the work variety. Brief descriptions identify the purpose of each. 


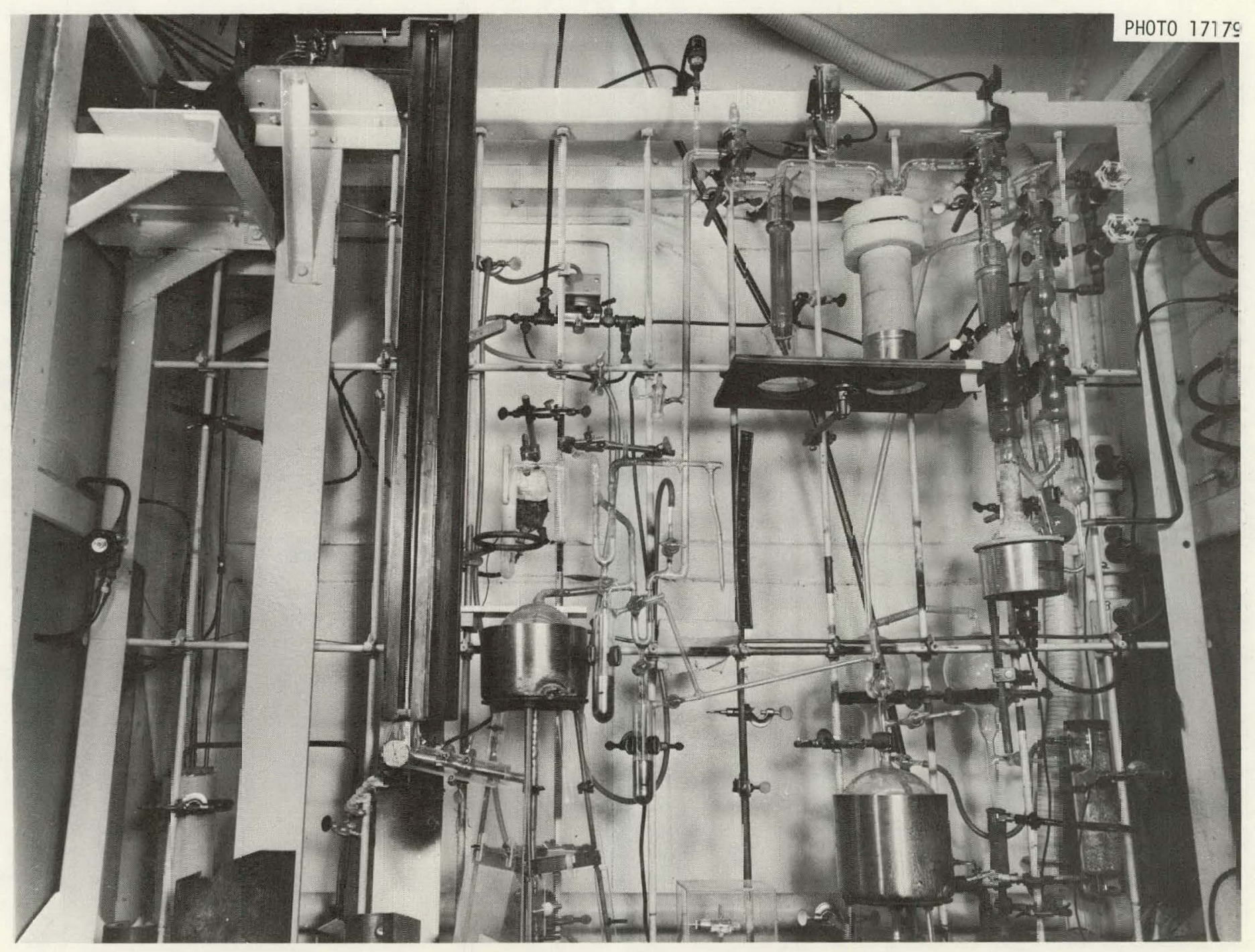

Fig. 1. A laboratory glass system for radon gas containing many typical components combined precisely to suit the desired experimental processing intended by the Chemistry Division. 


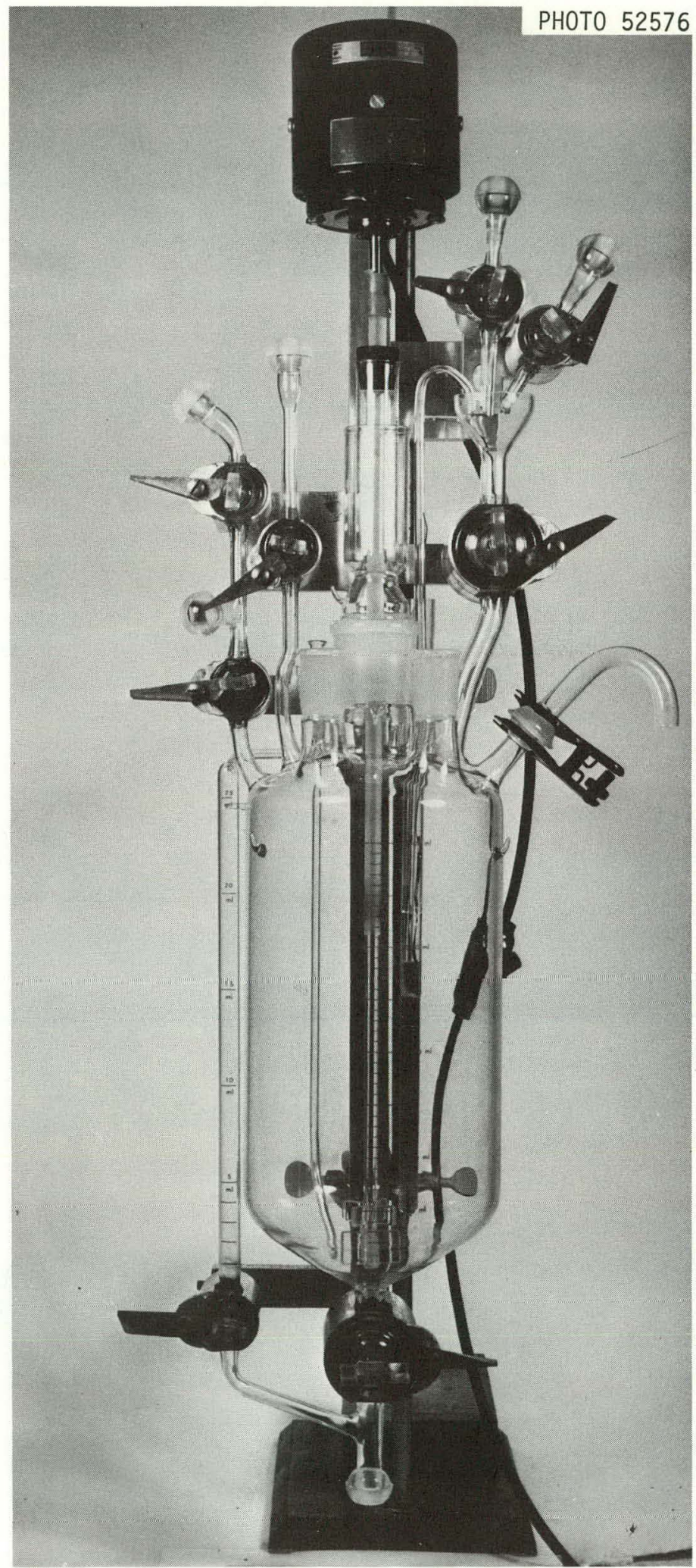

Fig. 2. Mixer-settler unit for first Transuranium Element Processing - Chemical Technology Division. 


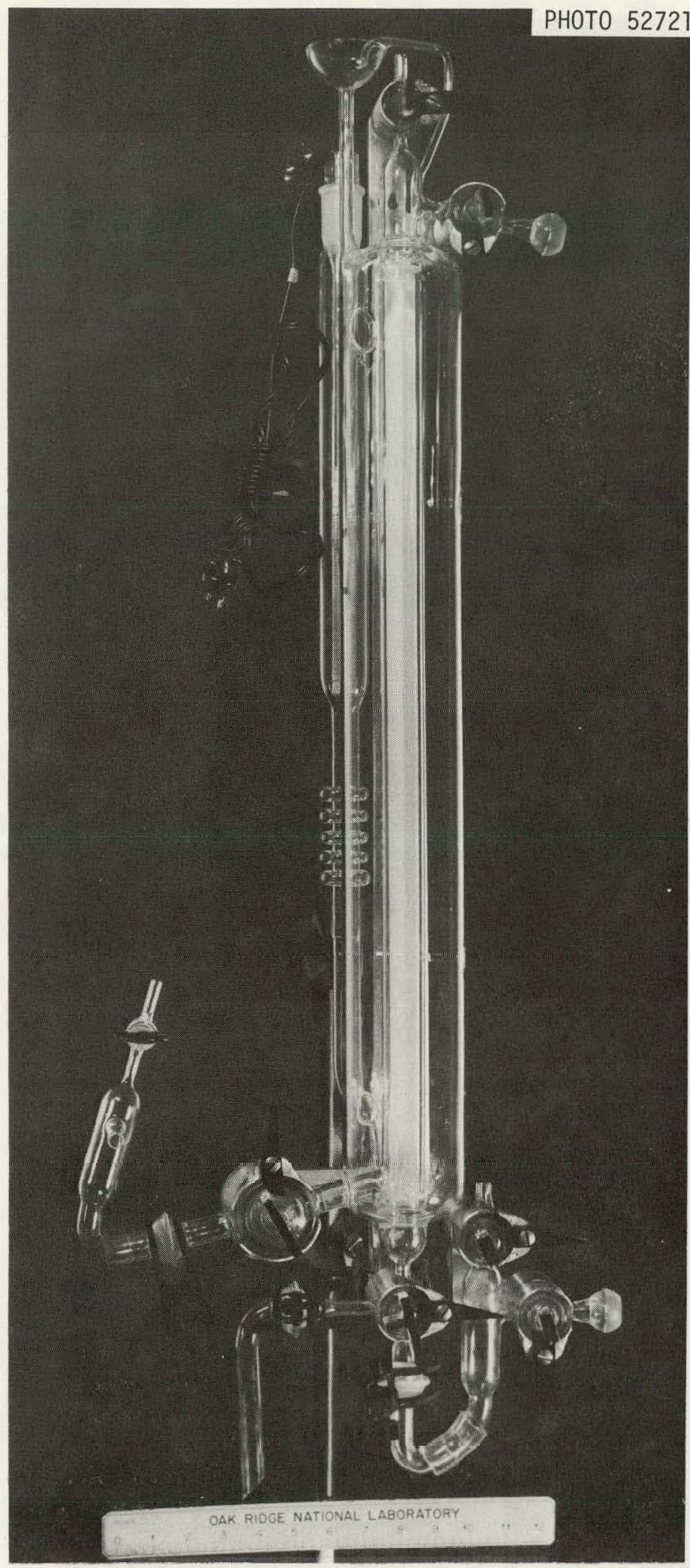

Fig. 3. Ion Exchange Column for first Transuranium Element Processing - Chemical Technolngy Division. 


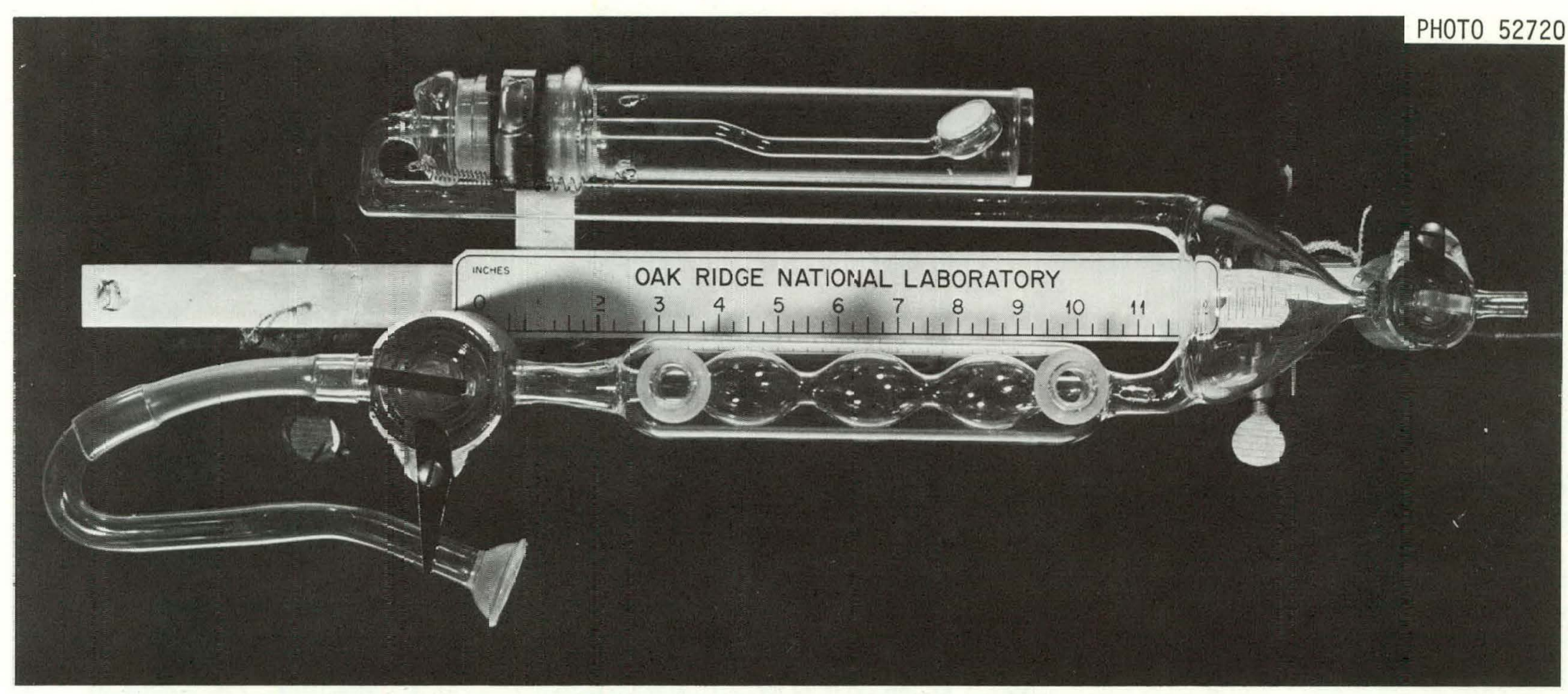

Fig. 4. Condensər-receiver for first Transuranium Element Processing - Chemical Technology Division. 


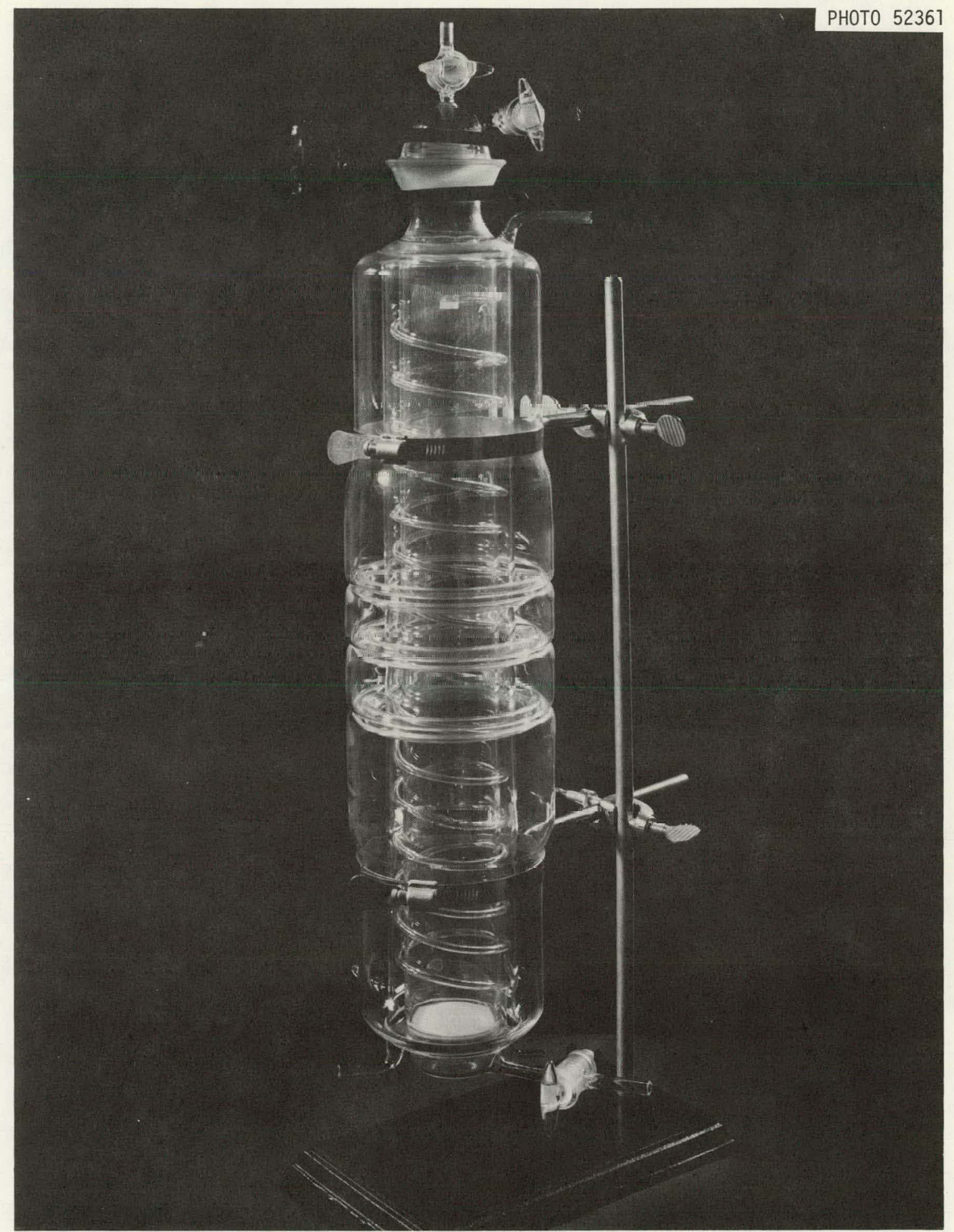

Fig. 5. Ion exchange column (for Analytical Chemistry Division) with a temperature control jacket, solution preheater, outer vacuum jacket, and bellows convolutions to limit stress buildup from temperature changes. 


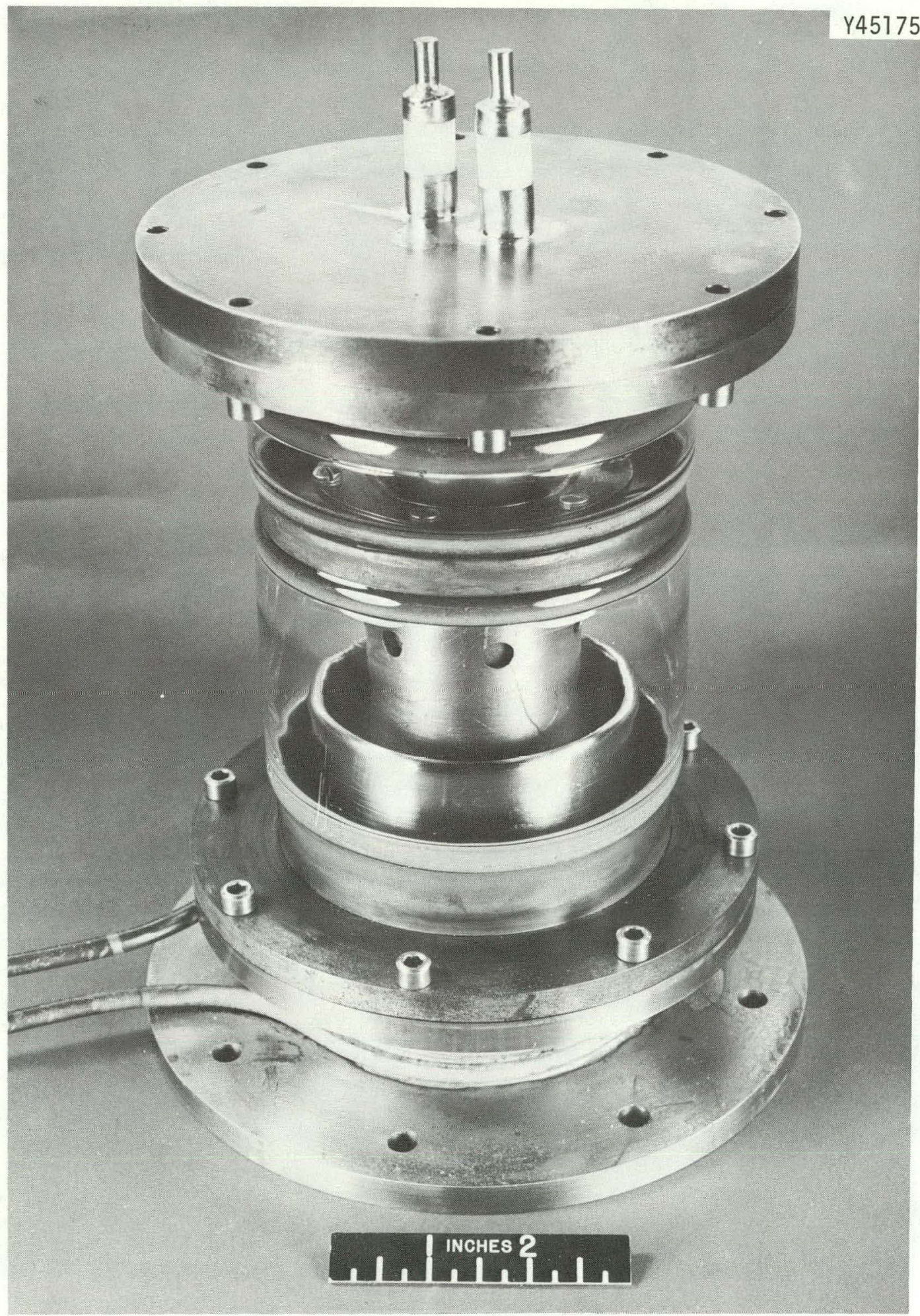

Fig. 6. Demountable Ion Gun for the Metals and Ceramics Division. An alignment tolerance of $0.1 \mathrm{~mm}$ and the visible $102 \mathrm{~mm}$ diameter kovar seals were factors for the glassblower to work out. 


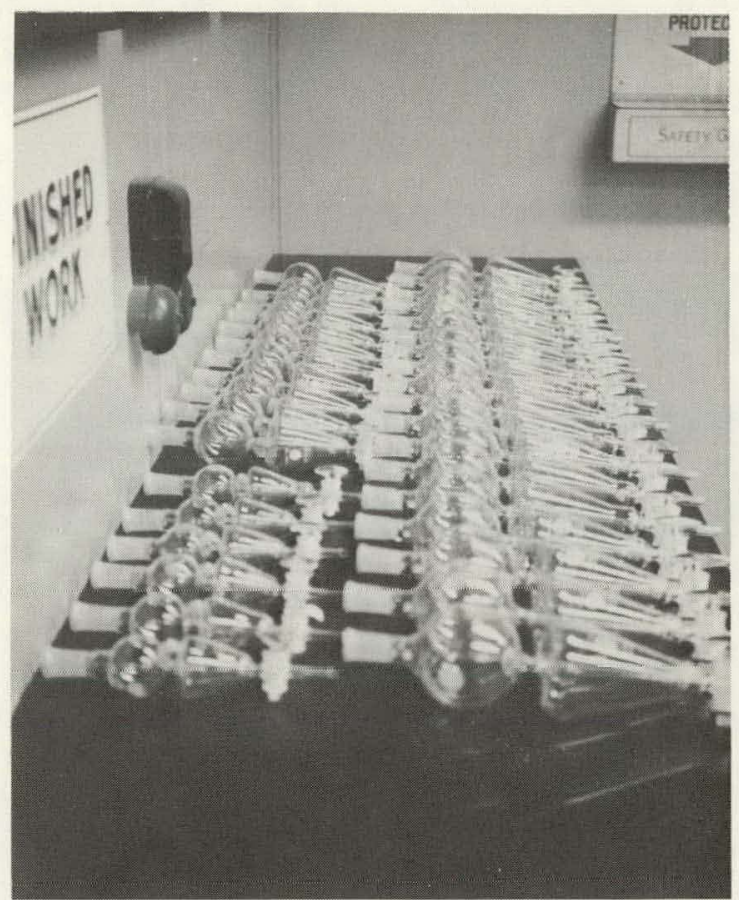

Fig. 7. Three dozen interchangeable, disposable units made for installation, operation, and removal by remote manipulator in a hot cell chemical system. Chemical Technology Division.

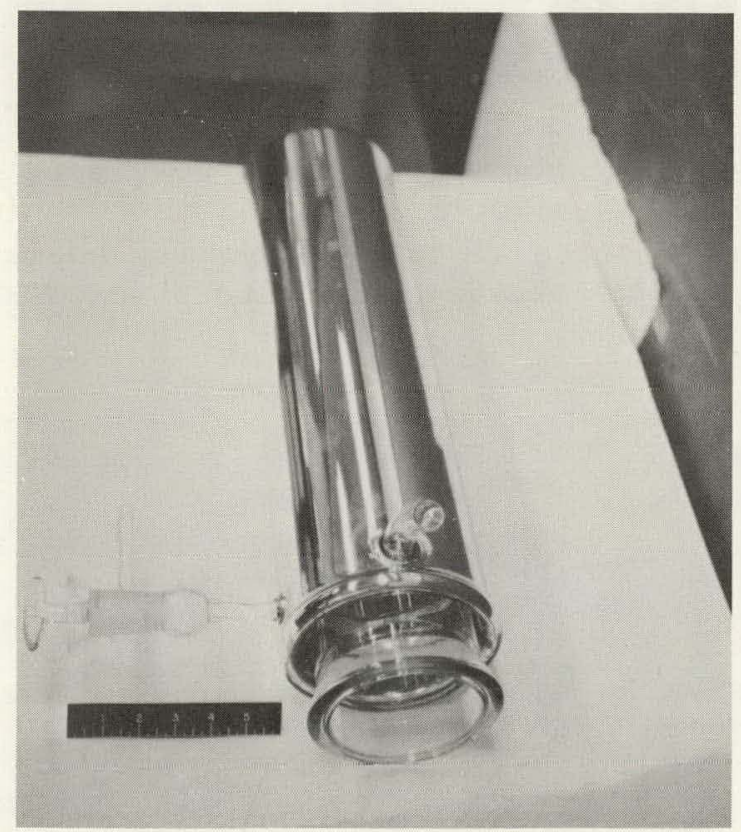

Fig. 8. Unusual nested, double dewar built to contain liquid nitrogen surrounding liquid helium and having interconnected insulating vacuum spaces. The Physics Division used such dewars in studies of radiation scattering at temperatures near absolute zero. 


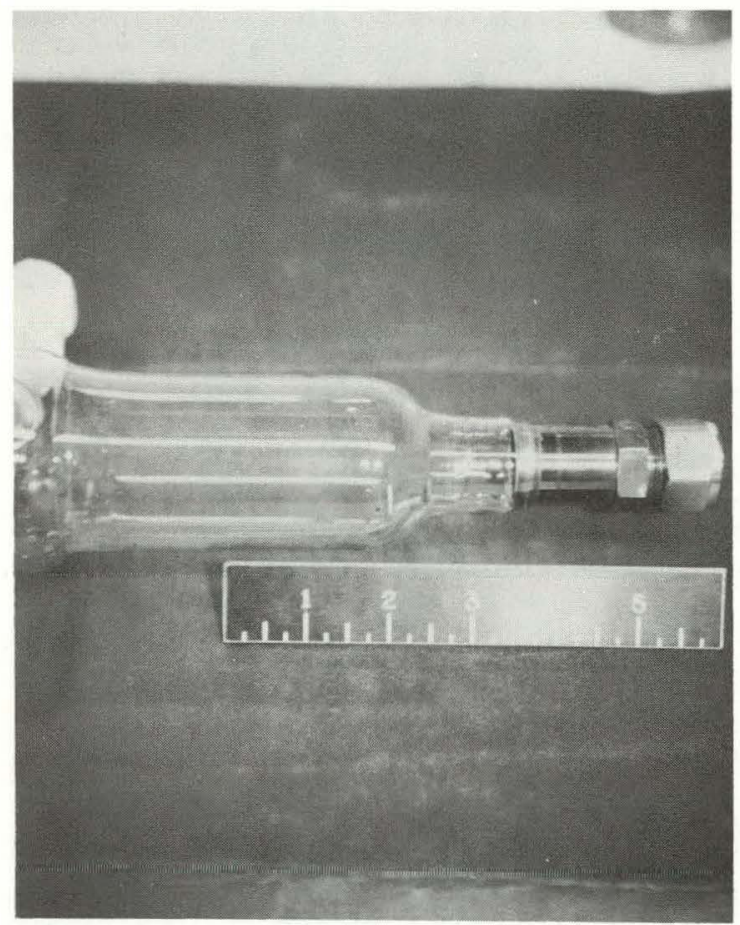

Fiy. 9. Ziscaloy II-to-glass scal developod for a chemical process requiring unusual corrosion resistance. Investigation of oxidation characteristics testing of wetting angles and check of final seal strength were development steps necessary. 
WORK DEFINITION, ,.. SCHEDULES,... QUALITY _ASSURANCE

The scientists and technical personnel who specify and request glassblowing service do so in many different ways:' The exceptional man is one who knows his exact job requirement parameters of operating pressures, temperatures; shock.resistance, chemical resistance; electrical resistance, ,.. physical dimensions.; and al so the gilasses with characteristics that are acceptable for the application and the best choice of system connections, seals; etc.. Occasionally, a.job will be overdesigned or overengineered for lack of specialized knowledge or consultation. Dimensional tolerances may be too restrictive for even skilled handwork. Excess material and redundancy may add cost without benefit in use. Most requesters will have some of their operating parameters and equipment general size in mind initially. Discussion. with the glassblower or chief glassblower develops the other necessary design information. so that the job can be properly done with least work. Others will have only the desired operation in mind and a rough flow sketch of a system, leaving most of the design-and-build work to the glassblower. Many others bring a retort or flask in one hand and a petcock in the other saying, "install this right at this spot," or "add a small condenser there." Most of the tougher and more involved job challenges the glassblower will discuss with,; or refer to, the chief glassblower. for assurance of best decisions on materials, methods, and processes.

Basic. scheduling of glass work is first in, first out: . Repair of breakage that delays an experimenter is allowed some priority. Divisions:can choose the order of their own jobs and Laboratory-set program priorities, are respected. The chief glassblower often corrects unbalance in the workloads of the three shops by moving jobs or manpower from one to another. Overall, the backlog range desired...is only...one to two weeks. Many small jobs and repair work are wanted immediately.

The chief glassblower is responsible for the work quality produced by his men, so he usually inspects all work visually, also 
checking any critical dimensions. The quality assurance originates with competent, conscientious, well-trained glassblowers and good materials. Guidance on other QA measures is supplied by Division and Department.. procedures. 


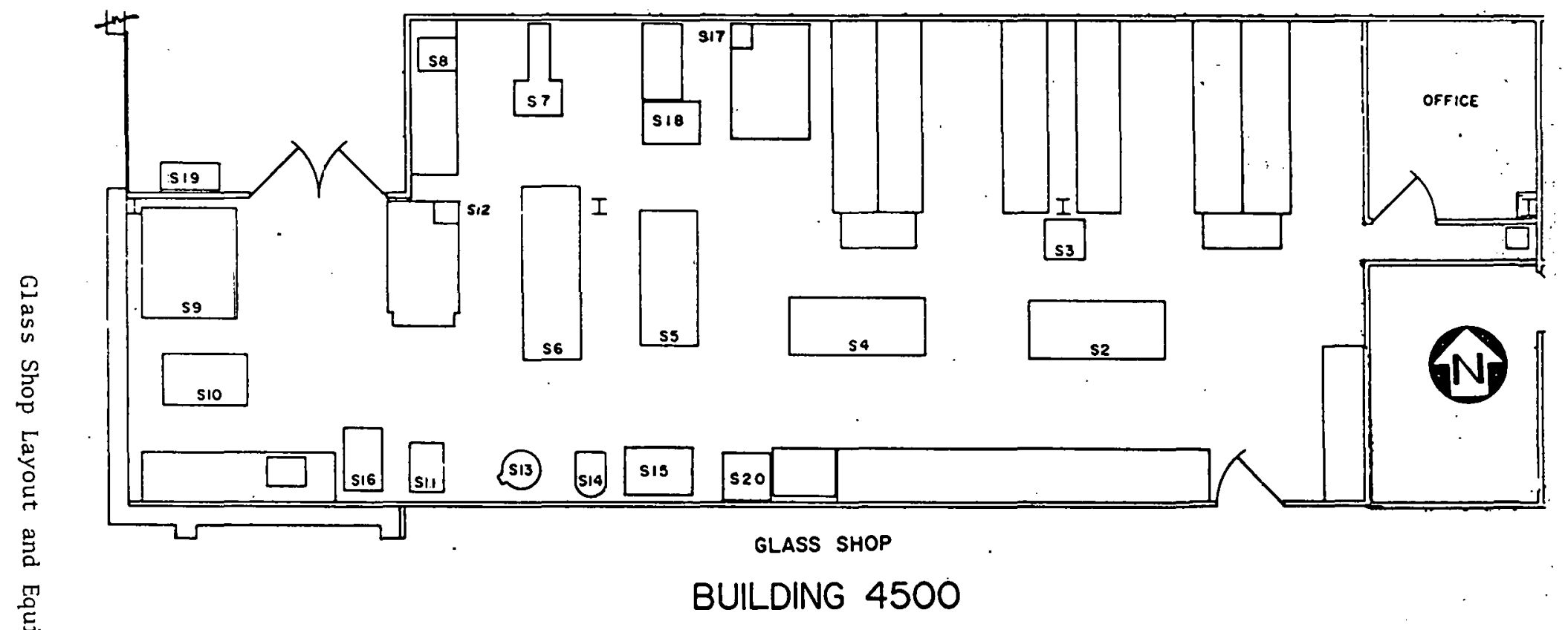

\section{Equipment list}

S2 Lathe, Glass, Universal \& Scroll Polarimeter

Lathe Glass, Universal \& Scroll

Lathe, Glass, Universal \& Scroll

Lathe, Glass, Universal

Drill, Radial, 1/2" Capacity

Furnace, $2000^{\circ} \mathrm{F}$. max.

Oven, $32 w \times 48 d \times 36 h, 1400$ F. max.

S10 Oven, $18 w \times 22 d \times 84 h, 1400$ F. max.
Sil Saw, Cut-off, slass

S12 Grinder, Belt $4^{\text {D }}$

S13 Grinding Wheel, 16" Dia.

S14 Drill Press. Pedestal,

S15 Latize, Glass, Vertical

S16 Sav, Cut-of6́, Glass

S17 Air Abrasive Urit

S18 High Vacuun System, $1 \times 10^{-4}$

s19 High Vacuum System, $1 \times 10^{-6}$

s20 Chucking Table, Vertical 
bal ANCE. TRIPLe beam, agate-bearing, $131 / 2$ IN. OVERALL LGTH, II $1 / 2$ IN. HT. MAX CAP. 201 GM. NO. 2640. CENCO OR VWR $12337-001$

BAL ANCE, IRIPLE BEAM. $55.610 \mathrm{GM}$ CAP., $0.10 \mathrm{GM}$ SENSITIVITY. NO. 7505, O HAUS SCALE COPP OR VWR $12340-050^{\circ}$

BALL-PESTLE, CLEAR PLEXIGLAS, 3/8 IN. DIA, 100 IN HEAT SEALEO PLASTIC BAG

BAR, STIRRING, MAGNET, ALNICO, SEALED IN TEFLON, REMOVABLE PIVOT RING. END CAPS OR PLUGS NOT ACCEPT$\triangle B L E, B E L-A R T$ F-37111

DIA X LGTH IN

$3 / 16 \times 7 / 8$ SARGENT $5-76497$

$5 / 16 \times 3 / 4$

MATHESON $60077-10$

$5 / 16 \times 117 \%$ FSCO $14-511-94$

$5 / 16 \times 13 / 4$ PREISER 22-2608-07

$3 / 8 \times 1$

BAR, STIRRING, MAGNET, ALNICO, SEALEO IN TEFLON, OCTAGONAL MOLDED PIVOT RING. END CAPS OR PLUGS NOT $\triangle$ CCEPTABLE

$$
\text { SILE IN. }
$$

$5 / 16$ (VWR $58948-138)$

$5 / 16$
$5 / 16$ IVWR $58948-138$
$5 / 16$

$5 / 16$ (VWR $58948-150)$
$5 / 16$ (VWR $58948-171$ )

LTH
1
1

BAR. STIRRING, MAGNETIC, SEALED IN HEAYY WALL PYREX BRAND GLASS, ALNICO MAGNET, NO. F-37101, BEL-ART, OR NO. 22-2510. PREISER

SILE IN.

$3 / 8 \times 13 / 8$

$9 / 32 \times 7 / 8$

BASE. FLUSH PLATE. THREADEO, 1 T/8 IN. DIA, 13 MM ROD OIA, 3/8-16 THD, CENCO 72125

BASE, FOOT. FLEXAFRAME, CASTALOY WITH SET SCREW AND 3 WOOO SCREWS, FOR ATTACHMENT TO TABLE. FLOOR. EIC. FISHER 14-666-25 OR MATHESON 61050-10 OR VWR 60095-005

BASKET, TEST TUBE, RECTANGULAR, DIAMOND WEAVE, CADMIUM PLATEO STEEL. SIZE $51 / 2 \times 4 \times 5$ IN. WILL 16602, PREISER 14-4150\%

BASKET, TEST TUBE, RECTANGULAR, EXPANDED ALUMINUM, SILE A, $5 \times 4 \times 4$ IN.. MATHESCN 61775-10 OR SCIENTIFIC PRODUCTS T1560-1

BASKET, TEST TUBE, RECTANGULAR, EXPANOED WELDED

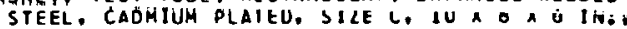
PRE ISER $14-4150-05$

BATH, WATER, SHALLOW FORM, TIANED COPPER, 10 IN. OIA. FISHER 15-518 OR WILL 28371

BEAD, SOLID GLASS, KIMBLE 13500 OR CORNING 7260

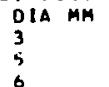

BEAKER, BERLELIUS, BOROSILICATE GLASS, TALL FORM HITH SPOUT, CORNING 1060 OR KIMBLE $14030^{\circ}$

CAP. ML

CAP.

300

500

1000

BEAKER. BERZELIUS, BOROSILICATE GLASS, TALL FORH, IITOUT SPOUT. CORNING 1040 OR KIMBLE 14020

CAP. ML

100

200

300
500

BEAKER, PORCELAIN GLAZEO, WITMOUT SPOUT $\begin{array}{lll}\text { CAP. ML } & \text { SILE } & \text { COORS NO. } \\ 580 & 3 & 04002\end{array}$

BEAKER. ELECTROLYTIC, BOROSILICATE GLASS, WITHOUT SPOUT, CORNING 1140 OR KIMBLE 14070

CAP. ML

180
Attachment $B$

UNIT PRICE STK PT CATALOG NO BUYER

EA $\quad 40.26 \quad 3 \quad 15-005-2051 \quad 36$

EA $27.03 \quad 2 \quad 15-005-2101 \quad 36$

BAG $2.85 \quad 125 \quad 15-005-3501 \quad 36$

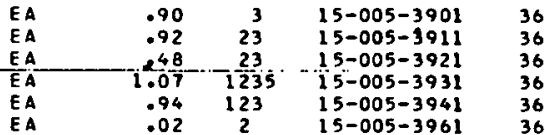

36

$\begin{array}{lllll} & & & & \\ \text { EA } & .71 & 2 & 15-005-3971 & 36 \\ \text { EA } & 1.70 & 2 & 15-005-3981 & 36 \\ & 1.08 & 2 & 15-005-3991 & 36\end{array}$

$\begin{array}{lllll}E A & .61 & 3 & 15-005-4051 & 36\end{array}$

EA $\quad .00 \quad 3 \quad 15-005-510136$

EA $\quad .66 \quad 23 \quad 15-005-5201 \quad 36$

EA $\quad .30 \quad 23 \quad 15-005-6001 \quad 36$

$\begin{array}{lllll}\text { EA } & .68 & 23 & 15-005-6051 & 36\end{array}$

EA $\quad 2.49 \quad 23 \quad 15-005-6071 \quad 36$

EA $13.85 \quad 3 \quad 15-005-7101 \quad 36$

$\begin{array}{lrrrr}\text { LB } & 17.12 & 23 & 15-005-8101 & 36 \\ \text { LB } & 11.29 & 13 & 15-005-8121 & 36 \\ \text { LB } & 8.51 & 1 & 15-005-8501 & 36\end{array}$

$\begin{array}{llllll}\text { EA } & .67 & 2 & 15-008-0502 & 36 \\ \text { EA } & .65 & 1 & 15-008-0522 & 36 \\ \text { EA } & .60 & 1 & 3 & 15-008-0542 & 36 \\ \text { EA } & .69 & 15 & 15 & 15-008-0602 & 36 \\ \text { EA } & 1 . .20 & 1 & 5 & 15-008-0622 & 36\end{array}$

$\begin{array}{lllll}\text { EA } & .43 & 2 & 15-008-0702 & 36 \\ \text { EA } & .45 & 23 & 15-008-0722 & 36 \\ \text { EA } & .60 & 2 & 15-008-0742 & 36 \\ \text { EA } & .61 & 2 & 15-008-0762 & 36\end{array}$

[A $3.90 \quad 3 \quad 15-008-1126 \quad 36$

EA $\quad .49 \quad 3 \quad 15-008-2102 \quad 36$ 
Att achment B

beaker, electrol Yitic, gorosillicate glass, hithout SPOUT. CORNING 1140 CR KIMBLE 14070

CAP: ML

250

-CONTINUED-

UNIT PRICE STK PT CATALOG NO BUYER

BEAKER, GRIFFIN, BOROSILICATE GLASS, LOW FORM, WITH SPOUT. WITH TEFLITN BONDED RIN, CCRNING NO. $1000-T$ CAP. ML

100

150

250 .

600

800
1000

BEAKER, GRIFFIN. BOROSILICATE GLASS, LOW FORM, WITH

SPOUT, CORNING 1000 OR KIMBLE 14000

CAP. ML

CAP. ML
1
5
10
20
30
50
100
150
250
600
600
800
1000
1500
2000

$4000 \quad 1$.

BeAKER, GRIFFIN, UYCOR GLASS, LOW FOR., WITH SPOUT,

NO. 11000. CORNING

CAP. AL

400

BEAKER, MICRO, BOROSILICATE GLASS, WITH LIP, NO, 1801 MICROCHEMICAL SPECIALTIES

CAP. ML

2 MISCO SCIENTIFIC $5-4020-8$

4. MISCO SCIENTIFIC 5-4020-0

BeAKER. SAMPLE CUP., 5 ML CAP.. FOR USE WITH BECKMAN

G PH METER. FISHER 11-505-i55 OR SARGENT S- 30134

BeAKER, PHILliPS, CONICAL, BOROSILICATE gLASS, $500 \mathrm{ML}$ CAP.: CENCO 14275 OR CORNING 1080

GEAKER, PQLYPROPYLENE. LT WT, POUR-OUT SPOUT,

LAPINE RZ1-25 MATHESON SCIENTIFIC SARGENT NO.
CAP. ML

$30 \quad 4040-05$ B2730-30 S-46880

$50 \quad(12 / P K G) \quad 4040-10 \quad 82730-50 \quad 54688 E$

\begin{tabular}{llll}
150 & $4040-20$ & $82730-100$ & $5-4688 F$ \\
150 & $4040-25$ & -- & $5-46886$ \\
\hline 50 & $4040-30$ & $82730-400$ & $5-46883$
\end{tabular}

$\begin{array}{llll}400 & 4040-30 & 82730-400 & 5-4686 J \\ 800 & 4040-35 & B 2730-600 & 5-46866 \\ 1000 & 4040-40 & - & -\end{array}$

Geaker, T.P.X., CLEAR POLYOLEFIN PLASTIC, NaLGE, No. 1203

CAP. ML

150
250 MATHESON $4041-20$
$25041-25$

BEAKER. STAINLESS STEEL. GRIFFIN LOW FORM. MS 15CA-2, TYPE 1

CAP. ML

129 MATHCSON 3754-10 ST!ENT. PRON. B2711-125 OR VHR 13977-010

250 MATHESON 3954-20. FSCO 2-5838 LAPINE O2149-03

BeAKER, STAINLESS STEEL, STRAIGHT SIDEs, ROUNo LIP

AT TOP. WITHOUT POUR-OUT SPOUT, MS 15-AA-2, TYPE 2

CAP. ML

500 MATHESON 3950-10. FSCO 2-584A

200 MATHESON 3950-20. FSCO 2-584

2000 MATHESON 3950-30, FSCO 2-584C

4000 MATHESON 3950-50, F SCO 2-584E

BEAKER, TEFLON, GRIFFIN, LOM FORM, MITM POUROUT LIP, SHOOTH SURFACED, SILE $290 \mathrm{ML}$, SARGENT S-4694E

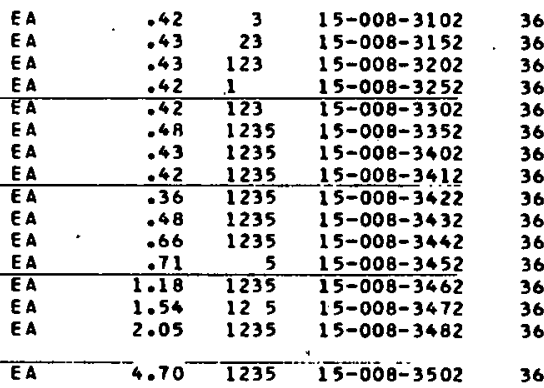

EA

$15.84 \quad 12$

$25-008-3702$

36
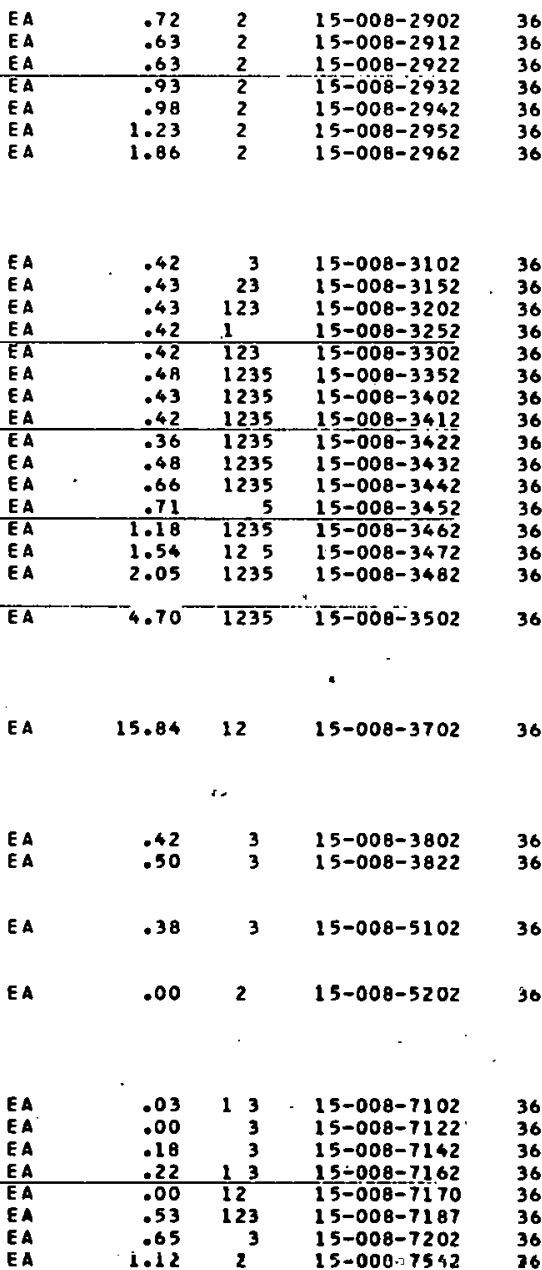

$\begin{array}{lllll}E A & .36 & 3 & 15-008-7802 & 36 \\ E A & 1.36 & 3 & 15-008-7812 & 36\end{array}$

EA $2.00315-000-0122 \quad 36$

EA $2.95 \quad 3 \quad 15-008-8142 \quad 36$

$\begin{array}{lcccc}\text { EA } & 2.82 & 23 & 15-008-8502 & 36 \\ \text { EA } & 2.88 & 1235 & 15-008-8532 & 36 \\ \text { EA } & 3.39 & 23 & 15-008-8572 & 36 \\ \text { EA } & 4.63 & 235 & 15-008-8592 & 36\end{array}$

EA $3.68 \quad 2 \quad 15-008-8602 \quad 36$ 
Attachment B

UNIT

PRICE STKPT CATALOG NO BUYER

BOTILE, SPECIFIC GRAVITY, ADJUSTED, VOLURE ADJUSTEO AT 20 DEG C. ENGRAVED HITH CORRESPONOING NO., MATHESON
CAP. ML
5
25
SARGENT S-9215A LAPINE 04026

BOTTLE, SPECIFIC GRAVITY, KIMAX, WITHERMCMETER + CAP, RANGE PLUS 14 TO 37 DEG $C_{\text {. }} 1 / 5$ OEG SUB-DIV. $10 \mathrm{ML}$ CAP.O KIMBLE 15123

BOTILE, SPECIFIC GRAYITY, LEACH WITH THERMOMETER AND CAP. RANGE PLUS 14 TO 37 OEG $C$. IN $1 / 5$ OEG AND CAP, RANGE PLUS 14 KIMBLE 15123

BOTTLE, SPECIMEN, GLASS, WIDE MOUTH, SQUARE, WITH NO. 28 BLACK MOLOEO SCREW CAP, 1 OL CAP. (30 ML) 12 /PKG. MATHESON 4363-10 OR VHR 16188-049

BOTILE, VACUUM, BOROSILICATE GLASS, SIL VERING IN THE VACUITY, 95 MM $10 \times 125$ MM OD $\times 300$ MM DEEP, OVERALL HEIGHT INCLUDING METAL BASE $145 / 8$ IN.. NQ. LG 7600 . LABGLASS

BOTTLE, VIAL, CAPSULE, WITH PLASTIC SCREW CAP
CAP. ML ODXHT MM MATHESON SARGENT NO.

\begin{tabular}{|c|c|c|c|}
\hline 15 & (4 DRAM) & $28 \times 57$ & $\begin{array}{c}63925-20 \text { S-83245F } \\
\text { OR VWR } 66012-022\end{array}$ \\
\hline & & & $S-83245 H$ \\
\hline
\end{tabular}

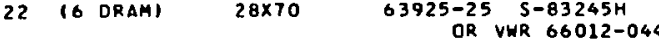

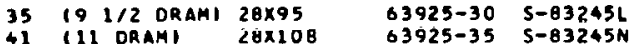

BOTILE, VIAL, CELLULOSE ACETATE, 5 ML CAP., 2 TO 2 1/2" HT $X$ 1/2" DIA. 125 MG/CM TO THE 2NO POWER walli. hith gasketeo nickel plateo steel cap. TRACERLAB E-52

BOTTLE, VIAL, FOR LIOUIO SCINTILLATION COUNTING, SILE $20 \mathrm{ML}$. WITH $22 \mathrm{MM}$ CAP. CAT. NO. 6000128, PACKARO INSTRUMENT CO

BOTILE, VIAL, FOR LIOUIO SCINTILLATION COUNTING. POL YETHYLENE, SILE $25 \mathrm{ML}$, POL YETHYLENE SCREW CAP, 500 PER CASE, NO. 6001075. PACKARD INSTRUMENT CO

BOTTLE, VIAL, GLASS, I ORAM CAP.. MITH PQLYETHYLENE CAP, G GROSS PER CASE, KI MBLE MO. 609651

BOTILE, VIAL. MIXING, POLYSTYRENE WITH POLYETHYLENE CAPS, I I/4 IN. DIA $X 3$ IN. LGTH, OU ML CAP.. NO CAPS, SPEX INOUSTRIES

HUIILE, VIAL. PLASTIĆ, S̈IZE I IN. $X 2$ IN.. WITH WHITE POLYETHYLENE SNAP-ON CaP, LERMER PLASTICS

BOTTLE, VIAL, POLYSTYRENE, WITH POLYETHYLENE SLIP-ON CAP. WITH THICK CONCAVE BOT TOM. SPEX INDUSTRIES $\begin{array}{llll}C A P . M L & \text { DIA IN. LGTH IN. } & \text { MFR NO. } \\ 2 & 112 \times x & 1111, & \text { NO SUB } \\ 5 & 112 \times & 3116, \text { NO SUB }\end{array}$

BOTTLE, VIAL, POLYSTYRENE, MITH POLYETHYLENE SNAP-ON CAP, SILE 44 MM $X$ B6 MM, 72 PER CASE, BROCKWAY GLASS CO

8OTILE, VIAL, POLYSTYRENE, ClEARSITE, RIGID SHOULOER, WITH POLYETHYLENE TYPE CAP, HHICH FITS ON OUTSIDE OF VIAL SHOULDER, CELLUPLASTIC CORP SILE IN.

$1 / 2 \times 1114$

$1 / 2 \times 2$

$3 / 4 \times 2$

$1 \times 2$

BOTTLE, VIAL, SPECIMEN, GLASS, SHORT STYLE, $1 / 2$ DRAM, $12 \times 35$ MM, NO, 60930, KIMBLE OR APPROVEO EQUAL

BOTTLE, VIAL, SPECIMEN, GLASS, SHORT STYLE, MACHINE MADE, UNIFORH ANO RETEMPEREO TO REMOVE STAINS, COMPLETE WITH BLACK MOLDEO SCREN CAP, NO. $60910-L$. KIMA1, E

$\begin{array}{ll}\text { CAP. DRAM } & \text { DO } \\ 1 / 2 & 12 \\ 1 & 15 \\ 2 & 17 \\ 8 & 25\end{array}$

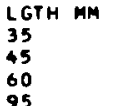

\begin{tabular}{|c|c|c|c|}
\hline $\begin{array}{l}\text { EA } \\
\text { EA }\end{array}$ & 2.00 & $\begin{array}{l}3 \\
3\end{array}$ & $\begin{array}{l}15-017-2205 \\
15-017-2225\end{array}$ \\
\hline EA & 19.22 & 2 & $15-017-2575$ \\
\hline EA & 19.22 & 3 & $15-017-2605$ \\
\hline$A$ & .00 & 1 & $15-017-3505$ \\
\hline & 28.63 & 1 & $15-017-4505$ \\
\hline$E A$ & .10 & 1235 & $15-017-4800$ \\
\hline & .06 & 123 & $15-017-4805$ \\
\hline EA & .07 & $\begin{array}{l}13 \\
23\end{array}$ & $\begin{array}{l}15-017-4810 \\
15-017-4815\end{array}$ \\
\hline
\end{tabular}

EA

CASE $\quad 35.09 \quad 2 \quad 15-017-5010 \quad 36$

EA

EA

EA

EA .132

15-017-5275 36

$\begin{array}{llll}.06 & 12 & 15-017-5505 & 36 \\ .08 & 23 & 15-017-5515 & 36 \\ .09 & 23 & 15-017-5525 & 36 \\ .18 & 1^{25} & 15-017-5565 & 36\end{array}$


Attachment B

BOTTLE, VIAL, SPECIMEN, GLASS, SCREM CAP, SHORT STYLE MACHIME MAOE. UNIFORM AND RETEMPERED TO REMOVE STAINS, COMPLETE WITH BLACK MOLOLO CAP, DRAM CAP.. 21 MH OD. 70 KM LGTH, NO. 60910. KImBe

BOT TLE. HASHING, CORNING 1660 OR KIMBLE 15135 CAP. ML
500
1000

Bot tLE. MASHING, POLYETMYLEAE, COMPLETE WITH DISPEMSING ASSY, 8 O2 CAP.. NO. AC-2030, SCHAAR OR NO. 10-\$160126. PRE I SER

BOT TLE. HASHING, POLYETHYLENE, 16 O2 CAP. $1500 \mathrm{ML})$ MATHESON 4653-15 OR SCIENT. PROD. B 7896-500 OQ VWR 16651-107

BOT TLE, WASHING, POLYETHYLENE, COL OR RED, COMPLETE WITH DISPENSING ASSY. MALGE NO. 2404

CAP. ML

NO. PER

250 (VHR 16651-722)

CASE

24

BOTTLE, WEIGHING. MIGH FORM. WITH SIT CAP STYLE GLASS STOPPER, KIMBLE ISI46

$\begin{array}{ll}\text { CAP. ML } & \text { S/T } \\ 12 & \text { SYOPPER } \\ 12 & 19 / 10 \\ 12 & 29 / 12 \\ 16 & 29 / 12 \\ 70 & 45 / 12\end{array}$

BOTTLE. WEIGHING. HIGH FORM, WITH SIT GLASS STOPPER, K BRAND GLASS, NO. 15145. KIMBLE

$\begin{array}{lll}\text { CAP. ML } & \text { S/T STOPPER } & \text { S12E MM } \\ 12 & 24 / 12 & 25 \times 12 \\ 16 & 24112 & 25 \times 50 \\ 30 & 29 / 112 & 30 \times 60\end{array}$

30

40112

$40 \times 80$

BOTTLE, WEIGHING, LOW WIOE FORM. MITH S/T GLASS STOPPER, K BRAND GLASS, $60 \mathrm{MM}$ ID, $30 \mathrm{MW} \mathrm{MT}$, NO. 15165. KI MBLE

BOTTLE, WIDE MOUTH, aMBER GLASS, RCUND, THREAOEO NECK, WITHOUT SCREM CAP, FOR BLACK ENAMELEO METAL CAP, CENCO 10355

CAP. OL

CAP NO.

SARGENT XS-83008

33

- SARGENT XS-630OC

38 VMR $16180-025$

BOTTLE, WIOE MOUTH. CLEAR GLASS, RCUND WITH MOLDED BLACK BAKEL ITE CAP, TOTAL HI INCL CAP NOT TO EXCEEO 5 5/16 IN.. OIA NOT TO EXCEED $27 / 16$ IN.. CAP. B OL SARGENT S-9185E OR VMR 16194-063

BOTTIE. HIDE MOUTH. CLEAR GLASS. RCUND MITH MOLDED BLACK BAKELITE CAP, BOTTLE NO. 17211 aNO CAP NO. BLACK BAKELITE CAP

CAP. O2

GLASS CONT

2 SARGENT S-91858

SARGENT $S-9185$

ASSN NO.

SCIENT PROD AC-4. BT505-48

16 SCIENT PROO AC-16.87305-70 70

32 PAEISEP 10-4286IOG VWR $16194-100$

38

63

BOTTLE, WIDE MOUTH, CLEAR GLASS, SCREW NECK, MITH HOL DEO DLACK BAKELITE GAP, BOTTLE NO, R-5isn, r.AP. NO. $\theta=5225$, SGA

$\begin{array}{ll}\text { CAP. } & \text { GLASS CON } \\ \text { OL } & \text { ASSN NO. }\end{array}$

V4R $16104-020$

MATHES ON 4361-20+4747-51
VWR $16104-063$

ASSN NO.

38

MATHESON $4361-30+4747-66-\frac{58}{70}$

MATMESON 148-593+4747-72

SCIENT PROD 67530-64*87505-83 --

UNI

PRICE STK PT CATALOG NO BUYER

\begin{tabular}{|c|c|c|c|}
\hline$E A$ & .12 & 2 & $15-017-5705$ \\
\hline $\begin{array}{l}E A \\
E A\end{array}$ & $\begin{array}{l}8.35 \\
9.02\end{array}$ & $\begin{array}{l}3 \\
3\end{array}$ & $\begin{array}{l}15-017-6515 \\
15-017-6525\end{array}$ \\
\hline$F \Delta$ & .38 & 23 & $15-017-0705$ \\
\hline$E A$ & .42 & 1 & $15-017-6755$ \\
\hline & . & . & \\
\hline $\begin{array}{l}E A \\
E A\end{array}$ & $\begin{array}{l}.78 \\
.83\end{array}$ & $\begin{array}{l}3 \\
3\end{array}$ & $\begin{array}{l}15-017-8785 \\
15-017-6795\end{array}$ \\
\hline $\begin{array}{l}E A \\
E A \\
E A \\
E A\end{array}$ & $\begin{array}{l}1.32 \\
2.35 \\
2.43 \\
2.75\end{array}$ & $\begin{array}{r}23 \\
3 \\
12^{3}\end{array}$ & $\begin{array}{l}15-017-7305 \\
15-017-7310 \\
15-017-7325 \\
15-017-7385\end{array}$ \\
\hline
\end{tabular}

EA

$\begin{array}{lrrr}1.55 & 23 & 15-017-7605 & 36 \\ 1.37 & 3 & 15-017-7625 & 36 \\ 1.51 & 3 & 15-017-7645 & 36 \\ 2.53 & 3 & 15-017-7680 & 36\end{array}$

EA $4.35 \quad 3 \quad 15-017-7805 \quad 36$

EA $\quad .07 \quad 2 \quad 15-017-87.05 \quad 36$

EA $\quad .00 \quad 2 \quad 15-017-8225$

$.11 \quad 12 \quad 15-017-8375$

$E$

E

$.0 B \quad 12$

$15-017-8405$

36

15-017-8425 36

$.07 \quad 2$

$15-017-846$

36

$17 \quad 123 \quad 15-017-8485 \quad 36$

.09
.06
.16
.03
.17
.36

$15-017-8488$

$15-017-8489$

$15-017-8491$

$15-017-8493$

$\begin{array}{ll}15-017-8494 & 36 \\ 15-017-8495 & 36\end{array}$ 
Attachment B

HULB, RUBEER, COMPLETE WITH VALVE, FOR BLOOD PRESSURE CUFF. NO. 6246-5088-01. TAYLOR INSTRUMENT CO. URE CUFF

RULB. RUBBER, DOUBLF ACTION, PRESSURE OR SUCTION, NON-BL OOMING OVAL SHAPED WITH HARO RUABER CHECK NON-BL DOMING, OVAL AT BOTH ENDS, FOR RUBBER TUBING CONN, NO. VALVES AT BOTH ENOS, FOR RUBBER TUBINC

BULB. RUBBER, OROPPING BOTILE, MEOICINE OROPPER, NO. 14-065, FISHER

CAP. ML

1 VHR $56311-027$

LGTH MM

B. RUBBER, DROPPING BOTILF, MEOICINE OROPPER PIPETTE, 2 ML OISPLACEMENT, 3 ML CAP.. NO. 23266. WILL CORP OR NO. 5-73135. SARGENT PREISER 13-9200

BULB, RUBBER, HEAVY DUTY, PEAR SHAPED, WITH REINFORCING RIRS. $1 / 4$ IN. BORE. BULB DIA $25 / 8$ IN. BULB LGTM 3 3/4 IN., CAD. 4 OL, NO. 57420-10 MATHESON, SARGENT S-73165. PRFISFR 13-9220-0

RULR. RUBBER, FOR USE WITH PIPETTES AND SYRINGES, WILL 23271

CAP. ML LGIH IN. DIA IN.

\begin{tabular}{|c|c|c|c|}
\hline 10 & $13 / 4$ & $11 / 16$ & $\begin{array}{l}\text { MATHESON } 5 T 410-05 \\
\text { PREISER 13-9220-01 }\end{array}$ \\
\hline 15 & 2318 & $11 / 4$ & FSCC $14-0708$ \\
\hline 75 & 4 & 2 & $\begin{array}{l}\text { PRFISFR } 13-9220 \\
\text { OR MAIH. } 57410-20\end{array}$ \\
\hline
\end{tabular}

GULB. RUBOER, HEAVY WALI NECK, FUR USE IITH PIPEITES - SYRINGES, FISHER 14-070 UR SARGENT S-73145C SYRINGES. FISHER 14-0IOTOR SAR

30 10 3 A

BUL 8. RUBBER. THOMPSON ACID PUMP, NO. AL-030. BURRELL CORP OR MATHESON 135-25

RURETTE, automatic, aCIO, Borosillicate glass, ro fit SCREW CAP REAGENT BTL, COMPLETE WITH PRESSURE SCREW CAP REAGENT BTL, COMPLETE WITH PRESSURE BULE. REBASE TUBE, SPECTAL SCRE HCA BUT NOT RTL CAP. ML VUR $17585-044$

HURETTE, AUTOMATIC, BLUE LINE EXAX, RETESTED WITH THRE -waY SIF STOPCDCK aND FILLIAG TUBE BENT UP-

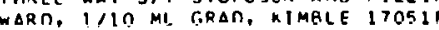
CAP. ML 25
50

QURETTF, AUTOMATIC, WITH S/, SIOPCCCK, 32 OL WIOE MOUTH BTL, RUBHER STCPPER, RUEBER BULB ANO CONNECTING TURE, 25 ML CAP.. NC. 6360-20. MATHESON $\checkmark$ WR OTIZISP, SARGENT S-1 1060

HURETTF, AUTMMATIC, LCFO POINT RURETTE ANO AIRINI.FT IUAE SFALED I $Y$ FULL LGTh S/T NO. 29/4? GROUNO STCPPER OF A $2000 \mathrm{ML}$ HTL, BURETTE GRAOUATFO IO $50 \mathrm{ML}$ IN $0.1 \mathrm{MI}$ NIVISINNS. CGNPLETE WITH ATL ANO RULB. CENCQI 15895. DREISED 10-8394/53 OR VWR $17590-066$

HUREITE, DISPENSING, BLUF LINE EXAX, WIT S/T SIMPCOCK, CORNING 2094 OF KIMBLE 17080.F

CAP. $M L$

250

RURETTE, GEISSLER, NORMAX, WITH STRAIGKT SIT SIOPCOCK, KIMALE 17031

SURDIVISICNS ML

$1>10$

$1 / 10$

CAD. ML

$1 / 5$

WURETTE, MICPU, AUTOMITIC, PYPEX GIASS, S/T INTERCHANGEABLE TIPS FOP CAL IRRATICA ANO DELIVERING. C.MANGEABLE TIPS FRP CAL IRRATICA AND DELIVERING, SILE $10 \mathrm{ML}$. ACF GLASS $5755=$ ?

MUPETIF. MICFO, RLUE IINE FXAX, ITH SIT IHRFF-HAY STIPC NCK, 1, 100 ML SUROIVISICA, $5 \mathrm{~mL}$ CAP.. NO.

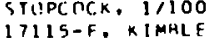


Attachment B

UNIT

PRICE STK PT CATALOG NO BUYER
BURETTE. MICRO, KOCH, STD TAPER JOINTS, WITH STOPPER AND TWO TIPS. 1/100 ML OIV. MO. 20-110. FSCO

$\begin{array}{lll}\text { CAP. ML VMR } & 17678-022 \\ 1 & \text { VWR } & 17678-044 \\ 2 & \text { VMR } & 17678-060\end{array}$

2
5 VWR $174778-044$

BURETTE, MICRO, PRECISION BORE, KIMBLE CAP. ML OIV ML

50.01

$17110-F$

5 With RESERVOIR

10 WITH LABCREST STOPCOCK

$17132-F$

OR VWR 17555-043

$17120-F$

OR VWR 17491-122

BURETTE, MANUFACTURING CMEMIST ASSN, NO. 1, WITH

FUNMEL TOP AND SIT OIAGONAL BORE STOPCOCK, SO ML

CAP.. 1/10 ML SUBDIVISIONS. NO. H-6300. MARSHAN

OR KIMBLE 17094-F

BURETTE, MOHR, RETESTEO, BOROSILICATE, KIMBLE 17019 CAP. ML

25 VWR 17409-020

BURETTE. PAECISION BORE, WITH LABCREST TEFLON STOPCOCK, 6/PKG, KIMBLE 17026-F

CAP. ML

CAP. ML

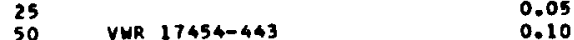

BURETTE, PRECISION BORE, BOROSILICATE GLASS, STRAIGHT BORE STOPCOCK, MITH SIT STOPCOCK STOPPER, CORNING 2130 OR KIMBLE 17027

CAP. ML

$\begin{array}{lll}\text { SO } & \text { VWR } & 17665-064 \\ 100 & \text { VHR } & 17665-086\end{array}$

STOPPER NO.

BURETTE, POLYSTYRENE, $1 / 10$ ML GRAO, haRSHAY

CAP. ML

25 A6-025

BURNER, ALCOMOL LAMP, BRASS, WITH RATCHET, DIA OF BOT TOM 90 MM. CAP. $150 \mathrm{ML}$. NO. 4-235, FISHER BOTTOM 90 MM, CAP. $150 \mathrm{ML}$, NO. 4-235. FISHER

BURNER ASSY, HYOROGEN-OXYGEN, FOR FLAME PHOTOMETER, MED BORE. BECKMAN 4020

BURMER, BUNSEN, MICRO MIXED, NATURAL ANO CYLINDER GAS, $21 / 2$ IN. HT, $1 / 4$ IN. DIA TUBE. WITH PGE TOP, MATHE SON $6875-20$ OR VHR 17911-002

BURNER, FISHER, BLAST, COMPRESSED AIR WITH GAS, NO. 3-910-5, FSCO, MATHESON 6675-20 OR VHR 18224-008

BURNER, FISMER, HIGH TEMP, ARTIFICIAL GAS, CHROMIUM PLATEO gRASS aNU WITITE HeTAL ALLOY, NO. 3-700. FSCO. MATHESON 6610-20 OR VMR 18220-062

BURNER, FORBES-BUNSEN, NATURAL GAS, NO, 3-917, FISHER OR MATHESON $6500-30$

CAN, STAINLESS STEEL, 22 GA, LEAK-PROOF, WITH LIO aND TWO HANOLES. 10 GaL CAP.

CANDLE, STANDARO, FOR USE IN JACKSCN TUROIOIMETER, RECOMMENOED BY AMER ICAN PUBLIC HEALTH ASSOC, TO PROVIDE STANDARD ILLUAINATION, NC. 28816. HILL SARGENT S-14535 OR PRECISION 69764

CANISTER ABSORBER, CYLINORICAL SHAPE, PERFORATED STEEL CONSTRUCTION, WITH 5/8 IN. LAYER OF ACTIVATED COCOANUT SHELL CHARCOAL TO MEET THE FOLLOUING REUUIREMENTS.

TVITY BY U S GOVERNMENT

ACCELERATEO CHLOROPICRIN TEST - NORMAL AIR

FLOW 25 CFM

RESISTANCE - $G 0.15$ IN

MODEL 7-CM. BARNEBY-CHENEY CO

CAP, BOTTLE, BLACK PLASTIC, SCREM TYPE, WITH VINYLITE LINER, 63 MM DIA. SARGENT S-961 ON $\begin{array}{rrrrr}\text { EA } & .00 & 3 & 15-035-4711 & 36 \\ \text { EA } & 8.40 & 3 & 15-035-4721 & 36 \\ \text { EA } & 14.00 & 2 & 15-035-4731 & 36\end{array}$

EA $32.27 \quad 3 \quad 15-035-4759 \quad 36$

EA $22.90 \quad 3 \quad 15-035-4761 \quad 36$

EA $22.81 \quad 3 \quad 15-035-6841 \quad 36$

$\begin{array}{lllll}\text { EA } & 2.77 & 3 & 15-035-4871 & 36 \\ \text { EA } & 3.60 & 3 & 15-035-4881 & 36\end{array}$

$\begin{array}{llrrr}\text { EA } & 9.21 & 3 & 15-035-5311 & 36 \\ \text { EA } & 8.55 & 23 & 15-035-5321 & 36\end{array}$

$\begin{array}{lrrrr}\text { EA } & 11.50 & 123 & 15-035-5431 & 36 \\ \text { EA } & 13.95 & 3 & 15-035-5451 & 36\end{array}$

$\begin{array}{lllll}\text { EA } & 22.79 & 3 & 15-035-6211 & 36 \\ \text { EA } & 33.00 & 3 & 15-035-6221 & 36\end{array}$

EA $\quad .00 \quad 3 \quad 15-038-5312 \quad 36$

EA $83.00 \quad 3 \quad 15-038-5412 \quad 36$

EA $\quad .00 \quad 3 \quad 15-038-5662 \quad 36$

EA $\quad 18.00 \quad 23 \quad 15-038-5712 \quad 36$

EA $\quad .00 \quad 2 \quad 15-038-5732 \quad 36$

EA $\quad .00 \quad 23 \quad 15-038-5812 \quad 36$

EA $\quad 45.12 \quad 2 \quad 15-042-4013 \quad 36$

EA $\quad .00 \quad 1 \quad 15-042-6013 \quad 26$

EA $\quad 15.30 \quad 3 \quad 15-043-2025 \quad 36$

EA $\quad .04 \quad 3 \quad 15-045-1034 \quad 36$ 
Attachment B

CLEANER, LIOUIO, AOUET, MUST BE NON-IONIC ANO NONTOXIC. FOR CLEANING LABORATORY GLASSHAPE ANO UTENSILS, I GAL GLASS REFILL BTL. EMIL GREINER PRE ISER MAN-19-090-03

Cle ANER, PIPE. 24 PER PKG, USED AS STOPCOCK CLEANERS, MATHESON 480-20 OR PREISER 10-7130

Cleaner, tUBe, BdUNo on mire, for Cleaning PIPETTES. STOPCOCKS AND PLUGS, CAPILLARY TUBING, ETC, NO.

3-642, FISHER, NO. 06906. WILL TUBE DIA $\begin{array}{lll} & & \text { MM } \\ \text { A } & \text { MATHESON } 6080-10 & 1.5 \text { TO } 3 \\ \text { B } & \text { MATHESON } 6080-25 & 2.5 \text { TO } 5 \\ \text { C SARGENT } 5-10055 \mathrm{C} & 5 \text { TO } 8\end{array}$

CLOTH, POLISHING, GAMAL, $12 \times 12$ IN., NO. 12-2828. FISHER

CLOTH, WOVEN, OUARTL, NO, C-100-28, 33 IN. WIDE, 100 YO ROLL. H I THOMPSON

COIL. HIGH FREQUENCY, SILE 14 IN. LGTH $X 2$ IN. DIA, WITH 6 FT. CORD AND PLUG. FOR TESTING VACUUM ASSYS FOR LEAKAGE AND EXCITING GE ISSLER TUBES, NO. 80730, CENCO OR VMR 63424-001

COLUMN. DISTILLING, VIGREAUX. MODIFIED, FRACTIONATING, PYREX GLASS, WITH MALE JCINTS WITH INTERATING P PYREX GLASS, WITH MALE JCINTS WITH INTER CHANGEABLE GROUNO JOINT, 24/40 MM LGTH, OVERALL

COLUMN. LaBoratory, SEPHADEX, Pharmacia fine CHEMICALS, IMC TYPE

$K \quad 15 / 30$
$K \quad 15 / 90$

K $26 / 40$ (JACKETED)

CONOENSER, ALLIHN, BULB TYPE, BOROSILICATE GLASS, AOAPTOR END TOOLEO FOR NO. 3 STOPPER, JACKET LGTH $400 \mathrm{MH}$. NO. 2420, CORNING

CONOENSER, ALLIHN, ORIP TIP, BOROSILICATE GLASS, CORNING 2480 OR KIABLE NO: 18260 JACKET LGTH MM OUTER AND INNER S/T JOINT NO. $200 \quad 19 / 38$.

$\begin{array}{ll}300 & 24 / 40 \\ 400 & 24 / 40\end{array}$

CONDENSER, ALLIHN, BOROSILICATE GLASS, INNER JOINT WITH 19/38 S/T JOINT. SIZE 250 MM. NO. 5945-05. ACE OR LABGLASS LG-4680

CONDENSER, FRIEOR ICHS, SPIRAL INNER TUBE, BOROSILICATE GLASS. APPROX LGTH 315 MM. NO. 2580 , CORNING

CONDENSER, GRAHAM, ORIP TIP, BOROSIllicate GLASS, CORNING 2560 OR KIMBLE 18360

JACKET LGTH MM OUTER ANO INNER S/T JOINT NO. 200 300
400

CONDENSER, LIEBIG, DRIP TIP, BOROSILICATE GLASS, CORNING 2400 OR KIMBLE 18140

JACKET LGTM MM OUTER ANU INNER SIT JOINT NO. 200 TACKET LGTH HM

$\begin{array}{ll}200 & 19138 \\ 300 & 24 / 40\end{array}$

CONDENSER, WEST, DRIP TIP, BOROSILICATE GLASS, OUTER AND INNER JOINT S/T 24/40, CURNING 2800 OR KIMBLE AND INNE 18190
JACKET LGTH MM

CONDENSER, WEST, IMPROVEO TYPE, BOROSILICATE GLASS, PLAIN AOAPTOR ENO TOOLED FOR NO 3 STCPPER, JACKET LGTH 200 MM. CORNING 2700 OR KIMBLE 18160

CONOENSER. PYREX GLASS. IN EXACT ACCOROANCE MITH ORNL SKETCH NO. A-3

CONE, CENTRIFUGe, BOROSILICATE GLASS, GRaDUATED 10 ML. IN I/1O ML DIV. MITH STD JOINTS AND MOOKS. H C CUMMER

CONE, CENTRIFUGE, BOROSICTCATE GLASS, PER ORNL OWG NO. SK-B-341, VENDOR TO FURMISH SAMPLES FOR APPROVAL PRIOR TO COMPLETION OF ORDER, OMG NO. 9980-2974. REV 1. CORNINC

UNIT

PRICE STK PT CATALOG NO BUYER

BT

PKG

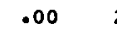

$15-054-0600$

36

.1323

$15-054-0630$

36

$\begin{array}{lrrrr}\text { FT } & .00 & 123 & 15-054-0650 & 36 \\ \text { FT } & .00 & 23 & 15-054-0652 & 36\end{array}$

EA $03 \quad 15-054-0030 \quad 36$

IFT $3.15 \quad 3 \quad 15-054-0965 \quad 31$

EA $\quad 11.12^{\prime} \quad 3 \quad 15-054-1570 \quad 36$

EA $10.37 \quad 3 \quad 15-054-2180$

$\begin{array}{lllll}E A & 32.87 & 2 & 15-054-2250 & 26\end{array}$

$\begin{array}{lllll}E A & 38.70 & 2 & 15-054-2255 & 26 \\ \text { EA } & 62.00 & 2 & 15-054-2260 & 26\end{array}$

EA $\quad 9.91 \quad 3 \quad 15-054-2450 \quad 36$

$\begin{array}{rrrrr}\text { EA } & 11.58 & 23 & 15-054-2460 & 36 \\ \text { EA } & 10.71 & 3 & 15-054-2485 & 36 \\ \text { EA } & 11.41 & 3 & 15-054-2490 & 36\end{array}$

EA $0.65 \quad 3 \quad 150542500 \quad 30$

EA $\quad 15.47 \quad 3 \quad 15-054-2530 \quad 36$

$\begin{array}{rrrrr}\text { EA } & 9.83 & 3 & 15-054-2630 & 36 \\ \text { EA } & 11.33 & 23 & 15-054-2635 & 36\end{array}$

$\begin{array}{lllll}\text { EA } & 9.87 & 3 & 15-054-2660 & 36 \\ \text { EA } & 9.59 & 3 & 25-054-2690 & 36\end{array}$

EA $\quad 9.93 \quad 23 \quad 15-054-2695 \quad 36$

EA $\quad 7.54 \quad 2 \quad 15-054-2900$

EA $\quad 3.84 \quad 2 \quad 15-054-2955 \quad 36$

EA $\quad 4.69 \quad 3 \quad 15-054-4100 \quad 36$

EA $4.50 \quad 3 \quad 15-054-4200 \quad 36$

EA $\quad 3.20 \quad 3 \quad 15-054-4225 \quad 36$ 
Attachment B

UNIT

PRICE STK PT CATALOG NO BUYER

CONNECTIOA, MOSE, SEALING, MADE OF BOROSILICATE GLASS, SERRATEO CONNECTIONS FOR RUBBER TUBING ON GLASS APPARATUS, UNIFORMLY TOOLED OF HEAYY WALL GLASS TAPERING SL IGHTLY FROM 8 TO 1
APPROX 45 MH. NO. LG-9010, LABGLASS

CONHECTOR, FLEXAFRAME, HARO ALUMINUM ALLOY, $1 / 2$ IN. DIA. ND. 61040-10, MA THESON OR WILL 25959-04

CONNECTOR, HOOK, FLEXAFRAME, WITH THUMBSCREW, NO. 14-666-18, FISHER, NO. 61065-10, MATHESON OR PREISER 14-3540-01

CONMECTOR, hOSE, BRASS, CHROME OR NICKEL PIATED. STRAIGHT, SERRATED, MALE THREAD

SILE IN. IPS

$1 / 4$ CONSOL IDATEO BRASS 44-301, BOEKEL 1882- 44

$3 / 8$ MATHESON 60320-10. FSCO 16-550A

CONMECTOR, hOSE, BRASS. CHROME OR NICKEL PLATED, 30 DEG ANGLE, MALE THREAD, 3/8 IN. IPS, NO, $9120 E$, VOL VERINE BRASS WORKS OR PREISER BO-4332-01

CONNECTOR, MULTI-CLUTCH. FLEXAFRAME, NO, 14-666-22, FSCO OR MATHESON 61056-10

CONNECTOR, OD, SILE B. NALGE 6150 OR SGA T-9500

CONNECTOR. TWISTCOCK, POLYETHYLENE, $33 / \theta$ IN. OVERALL LGTH. TUEE CONNECTION $7 / 8$ IN. LG. TAPES 3/8 IN. TO 114 IN.. SEALS AGAINST 30 PSI, NO, 27692 , WILL 174 IN-. SEALS AGA INST

CONTAINER. LIOUIO GAS, SIZE 25 LITER, NO. S-25, - stanoard air co

CONTAINER, Plastic, flexible, Souare, with LIO, for FREEZER AND REFRIGERATOR. NEATHAY PRODUCTS

FREEZER AND REFRIGERATOR, NEATHAY PRODUCTS
CAP.
CAT. NO.

CONTAINER, Plastic, $1 / 4$ IN. ID $\times 31 / 2$ IN. LONG, EACH CONTAINER TO CONSIST OF ONE $31 / 2$ IN. LGTH OF PLASTIC TUBING AND TWO CLOSURES. TIROS PLASTIC CORP

CONTATNER, PLASTIC, $5 / 8$ IN. $10 \times 3$ 1/2 IN. LGTH, EACH CONTAINER TO CONSIST OF ONE 3 IN. LGTH OF PLASTIC TUBING. ANU TMO ClOSURES. TIROS PLASTIC CORP

CONTAINER, PlaStIC, 3/4 IN. ID $\times 6$ IN. LONG, EACH CONTAINER TO CONSIST OF ONE 6 IN. LGTH OF PLASTIC TUBING AND TMO ClOSURES. TIROS PLASTIC CORP

CONTAINER. POLYETHYLENE, TALL FORM, 11 LITER, $479 / 16$ IN. TALL. T/8 IN. MAX ID, 83 MH BUTTRESS THREAO AND CAP. MS 15-AC-15A

CONTAINER, PLASTIC, EACH CONTAINER to CONSIST OF ONE 6 IN. LENGTH OF PLASTIC TUBING ANO TMO CLOSURES. TIROE PLASTIC TIORP

DIA IN 1

CONTAINER, MASTE, POLYETHYLENE, APPAOX DIM. TOP DIA $3 / 16$ IN.. BOTIOM DIA 7 IN. HT 10 IN.. MS 15-AC-

COLOR MAXEY MHITE

YELLOM

CONTRO, MICRO, DROP-SCALE PIPETTE. GROUND GLASS GYAIHGG: SIIE n.5 U. MO. 290-A, MIGROCHEMICAL SPECIALTIES

COUNTER, HANO TALLY, READS TO 9999 , HITH ZERO REVERTIHC KNOB ON SIDE. SATIM NICKEL FINISH. NO, 4481, WILL. FISHER 7-905. LAPINE 96-71 OR SARGENT S-23285 OR VHR 14395-010

COVER, CONBUSTRON, CERAMIC, FOR COMBUSTRON MODEL 120 - MODEL 2-50, BURRELL CORP 24-406

COVER, CRUCIBLE, NICKEL, Flat STYLE, No, 6-025, FISHER

FOR CRUCIBLE MM

30 PREISER 11-7170/45. LAPINE 09896-02

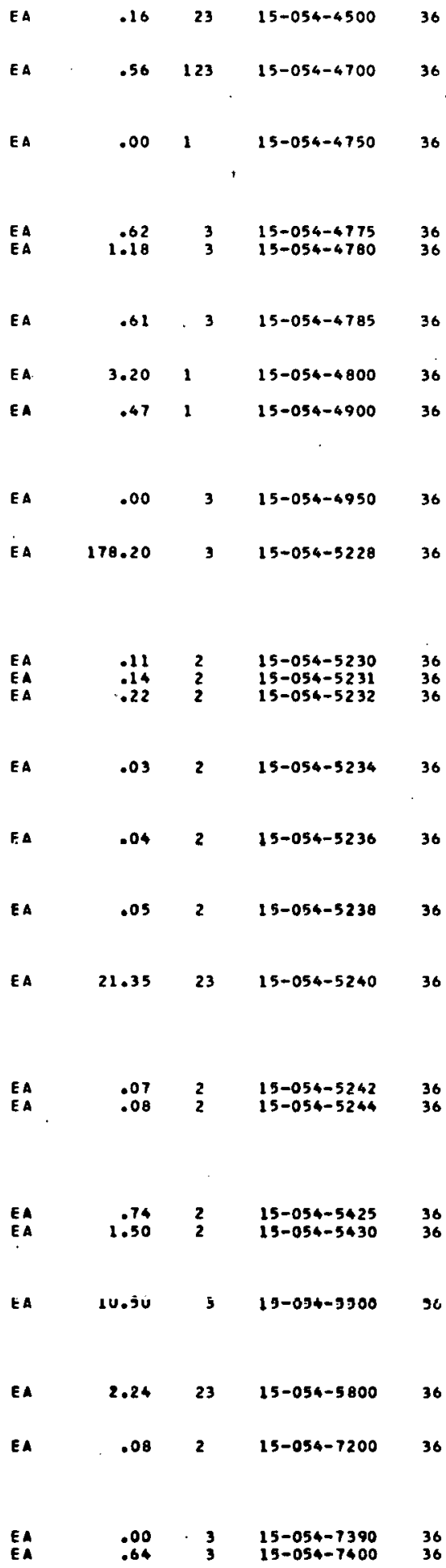


Attachment $B$

ELECTRODE, GRAPHITE, SPECTROGRAPHIC, HIGH PURITY, SOL IO ROD, MS 15-AC-7D, TABLE 1

DIA IN.

118

LGTH IN.

ELECTRODE, GRAPHITE, SPECTRCGRAPHIC, PYRO-CARRIER, PECIAL PURITY. PREFORMED. MS 15-AC-12G FIGURE

TYPE

a ISPECIAL INSPECTION REQUIREDI

CRATER

F ISPECIAL INSPECTION REQUiREoI

COUNTER

ELECTRODE

M ISPECIAL INSPECTION REQUIREDI

CRATER

FILM, POLYSTYRENE, 0.001 IN, THK, 2 IN. WIDE, I LB ROLL

FILTER APPARATUS, FISHER FILTRATOR, YACUUM CONTROL VALVE, COMPLETE WITH BASE, GLASS BELL. FUNNEL AND RUBBER STOPPER, BUT WITHOUT BEAKER, NO. 9-7B8, FSCO OR VWR 281B2-006

FILTER, DEMISTER, STAINLESS, 6 IN, DIA $\times 6$ IN. THK, MESH, ANY 300 SERIES. OTTO H YORK CO

FILTER. DISC, GLASS FIBER, 100 PER BOX, NO. 34-643. BURRELL CORP

SIIE

CM

2.1 IVWR $28496-751)$

FOR GOOCH CRUCIBLE

3

FILTER. DISC, GLASS FIBER, 100 PER BOX, FSCO 9-873-B

FILTER, STICK. MICRO, BOROSILICATE GLASS, 10 MM SIZE.

FLASK. BOILING, BOROSILICATE GLASS, FLAT BOTTOM,

RING NECK, WICKER PROIECTOR, CAP, $1000 \mathrm{ML}, \mathrm{NO}, 7$ STOPPER, CORNING 4220 OR KIMBLE 25170

FLASK, BOILING, BOROSILICATE GLASS, FLAT BOTTOM, SHORT NECK, S/T JOINT NO. 24/40, NO. 4100, CORNING OR KIMBLE 25055

CAP. ML

250

FLASK, BOILINC, BOROSILICATE GLASS, FLAT BOTTOM. VIAL MOUTH. CORNING 4060 OR KIMBLE 25000 LAP. ML

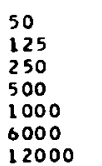

STIPPDER

NO.

1
1
4
5
7
10

FLASK, BOILING, BOROSILICATE GLASS, ROUNC BOTTOM, LONG NECK

CAP..ML S/T JOINT NO. CORNIAG NO. KIMBLE NO.

100

300

500

1000
2000

19138

24140

$24 / 40$

$2 4 \longdiv { 4 0 }$

$29 / 42$

$29 / 42$

CORNING NO. KIMBLE NO. 93245 25275

LASK, BOILING, BOROSILICATE GLASS, ROUNC BOTTOM, SHORT NECK, S/T JOINT 24/40, CORNING 4320 OR KIMBLE

25285

CAP. ML

100

200

250

300
500

1000

FLASK, BOILING, BOROSILICATE GLASS, ROUNC BOTTOM,

SHORT NECK, S/T JOINT NO. 29/42, CAP. $1000 \mathrm{ML}$, NO.

$\$ 321$ CORNING OR XIMALE 25290

UNIT PRICE STK PT CATALOG NO BUYER

$\begin{array}{lllll}\text { EA } & .65 & 3 & 15-080-4604 & 91 \\ \text { EA } & 1.03 & 1 & 3 & 15-080-4612\end{array}$

$\begin{array}{lll}.11 & 2 & 15-080-5025\end{array}$

EA

ROLL $2.22 \quad 3 \quad 15-084-2002 \quad 36$

EA $\quad 40.04 \quad 3 \quad 15-084-2302 \quad 36$

EA $\quad 12.21 \quad 2 \quad 15-084-2362 \quad 36$

BOX $\quad .76 \quad 5 \quad 15-084-2402 \quad 36$

B OX $\quad 1.90 \quad 5 \quad 15-084-2452 \quad 36$

EA $\quad 3.53 \quad 3 \quad 15-084-2702 \quad 36$

EA $\quad 3.55 \quad 12 \quad 15-087-1003$

EA $\quad 2.83 \quad 23 \quad 15-087-1108 \quad 36$

$\begin{array}{lrrrr}\text { EA } & 1.24 & 3 & 15-087-1303 & 36 \\ \text { EA } & .90 & 3 & 15-087-1306 & 36 \\ \text { EA } & .66 & 1 & 15-087-1309 & 36 \\ \text { EA } & 1.33 & 13 & 15-087-1312 & 36 \\ \text { EA } & 1.18 & 3 & 15-087-1315 & 36 \\ \text { EA } & 4.63 & 3 & 15-081-1318 & 36 \\ \text { EA } & 0.37 & 23 & 15-087-1321 & 36\end{array}$

$\begin{array}{lrrrr} & & & \\ \text { EA } & 3.16 & 23 & 15-087-1503 & 36 \\ \text { EA } & 2.66 & 23 & 15-087-1508 & 36 \\ \text { EA } & 3.92 & 3 & 15-087-1518 & 36 \\ \text { EA } & 2.02 & 23 & 15-087-1521 & 36 \\ \text { EA } & 5.02 & 3 & 15-087-1603 & 36 \\ \text { EA } & 4.95 & 3 & 15-087-1608 & 36\end{array}$

$\begin{array}{lcccc}\text { EA } & 2.28 & 2 & 15-087-1803 & 36 \\ \text { EA } & 2.46 & 3 & 15-087-1805 & 36 \\ \text { EA } & 2.42 & 12 & 15-087-1808 & 36 \\ \text { EA } & 2.45 & 2 & 15-087-1811 & 36 \\ \text { EA } & 2.60 & 2 & 15-087-1814 & 36 \\ \text { EA } & 3.52 & 2 & 15-087-1815 & 36 \\ & & & & \\ & & & & \end{array}$


Attachment B

UNIT PRICE STK Pt Catalog no buter

FLASK, BOILING, BOROSILICATE GLASS, ROUNO BOTTOM, SHORT RING NECK. CORNING 4260 OR KIMALE 25270 CAP. ML

200

500

1000

2000
5000

FLASK, BOILING, BOROSILICATE GLASS, ROUNO BOTTOM,

VIAL MOUTH, CORNING 4280 OR IIMBLE 25200

CAP. ML

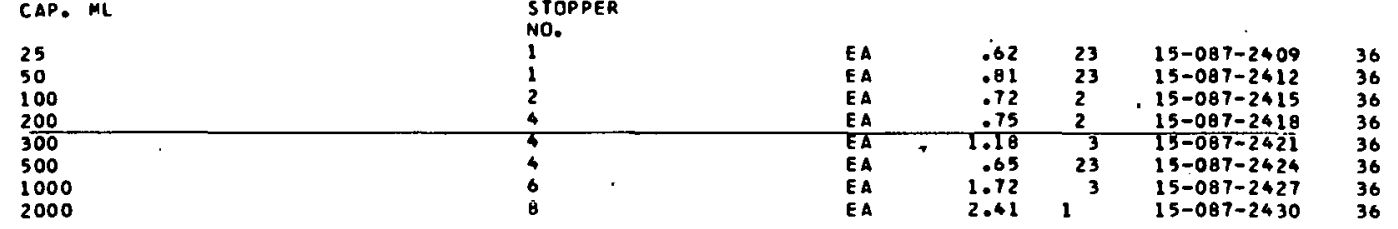

FLASK, BOILING, VITREOSIL, PLAIN, ROUNO BOTTOM, TRANSPARENT. THERMAL SYNDICATE LTO

TRANSPARENT

CAP.

250
500

FLASK, CULTURE, 250 ML, WITH SCREW CAP, 20 PKG PER CASE, NO. 3024 . FALCON PLASTIC

FLASK, OISTILLING, GOROSILICATE GLASS, CLAISEN, CAP. $230 \mathrm{ML}$. CORNING 4740 OR KIMBLE $26070^{\circ}$

FLASK, DISTILLING. BOROSILICATE GLASS. NO. 4620. CORNING OR KIMBLE 26015

50

125
250

1000
CAP. ML

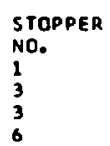

FLASK, DISTILLING, BOROSILICATE GLASS, THO MECK, SIT JOINTS. CORNING 4967

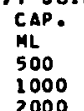

$$
\begin{array}{ll}
\text { JEINT } & \text { NC. } \\
\text { CENTER } & \text { SIDES } \\
34 / 45 & 24 / 40 \\
34 / 45 & 24 / 40 \\
45 / 50 & 24 / 40
\end{array}
$$

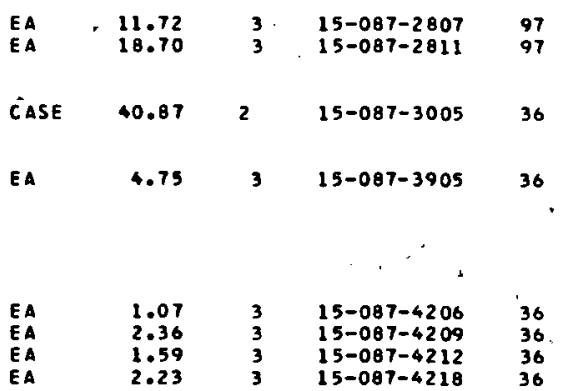

FLASK, OISTILLING, BOROSILICATE GLASS, TWO NECK, CAP. $3000 \mathrm{ML}$. S/T JOINTS, CENTER JJINT 29/42, SIDE JOINT 24/40. LABGLASS LG7290-318 OR ACE 6925-36

FLASK, DISTILLING, BOROSILICATE GLASS, THREE NECK,

SIT JOINTS JOINT NO. MFR ANO NO.
CAP. ML JOIN

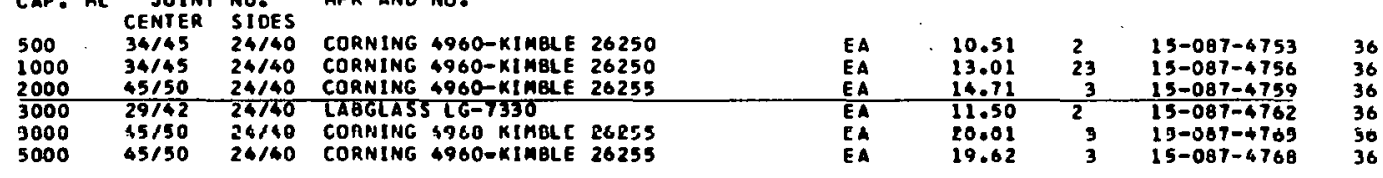

FLASK, DISTILLING. BOROSILICATE GLASS, THREE NECK,

ANGLE TYPE, S/T JOINTS

CAP. JOINT NO. CORNING KIMBLE

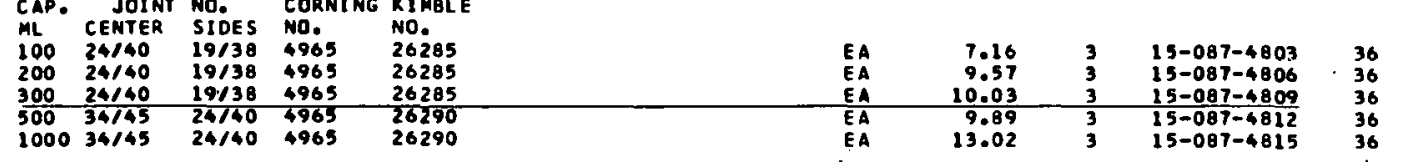

FLASK, ERLENMEYER, BOROSILICATE GLASS, NARROW MOUTH, CORNING 4980 OR KINBLE 26500

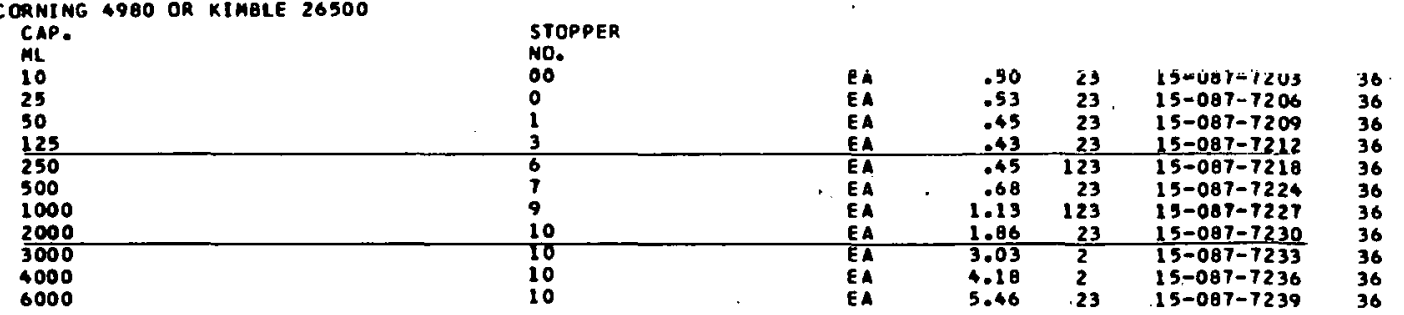


Attachment B

UNIT PRICE STK PT CATALOG NO BUYER

FLASK, ERLENMEYER, BOROSILICATE GLASS, MARROW MOUTH, WITH PENNY HEAD. SIT STOPPER, NO. 5020 . CORNING OR KIMBLE 26600

OR KIMBLE 26600
CAP.
ML
10
25
50
125
250
500
1000

STOPPER

ASK, ERLENMEYER, BOROSILICATE GLASS, NARROW MOUTH, SOCKET JOINT

CAP. ML JOINT NO. MFR ANO NO.

125 19/38 LABGLASS LG-7750

NO.

9 EA $1.86 \quad 3 \quad 15-087-7595$

$\begin{array}{llllll}16 & E A & 1.90 & 23 & 15-087-7603 & 36 \\ 19 & \text { EA } & 1.96 & 23 & 15-087-7605 & 36\end{array}$

\begin{tabular}{rrrrrr} 
& EA & 2.78 & 23 & $15-087-7607$ & 36 \\
\hline & EA & 2.56 & 123 & $15-087-7609$ & 36
\end{tabular}

32

$\begin{array}{lrl}3.45 & 23 & 15-087-7611\end{array}$

36

CORNING 5100 OR KIMBLE 26650
CAP.

CAP.
ML
500
1000
2000

STOPPER
NO.
10
11
13

$1.91 \quad 3 \quad 15-087-7816 \quad 36$

2000

$\begin{array}{ccccc}\text { EA } & .69 & 123 & 15-087-7856 & 36 \\ \text { EA } & 1.37 & 23 & 15-087-7859 & 36 \\ \text { EA } & 1.75 & 2 & 15-087-7861 & 36\end{array}$

FLASK. FILTERING, BOROSILICATE GLASS, heaVy HaLl.

WITH TUQULATION, CORNING 5340 OR KIMBLE 27060
CAP.
STOPPER

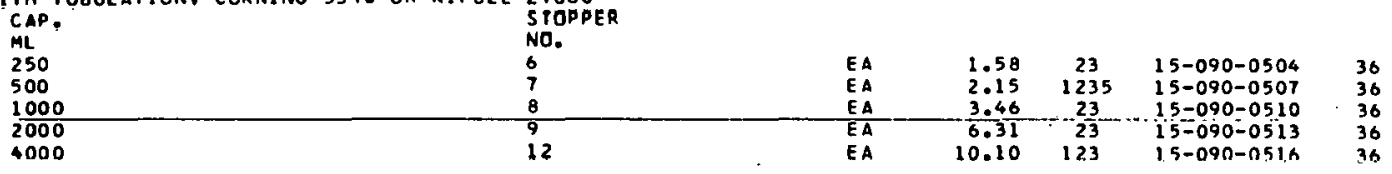

Flask. FILTERING. BoroSILICATE glass. meavy WALL.

WITH REPLACEABLE TUBULATIONS. NO. 5341, CORNING OR KIMBLE 27065

$\begin{array}{ll}\text { CAP. } & \text { STOPPE } \\ \text { ML } & \text { NO. } \\ 250 & 6 \\ 500 & 7 \\ 1.000 & 8\end{array}$

FLASK, FILTERING, BOROSILICATE GLASS, MICRO, HITH TUBULATION, CORNING 5360 OR KIMBLE 27060

CAP.

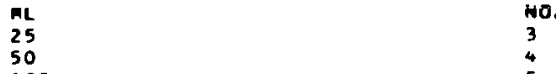

125

STOPPER

3
4

$\begin{array}{lll}E A & 1.94 & 23 \\ E A & 1.44 & 23\end{array}$

$1.50 \quad 23 \quad 15-090-0713$

36

FLASK, IODINE DETERMINATION. BOROSILICATE GLASS, MITH

S/T STOPPER NO. 22, CORNING 5400 OR KIPBLE 27200

CAP. ML

125 $\begin{array}{lllll}\text { EA } & 3.37 & 3 & 150901504 & 90 \\ \text { EA } & 5.54 & 3 & 15-090-1508 & 36\end{array}$

FLASK, KJELDAHL, BOROSILICATE GLASS, ROURD BOTTOM, LONG NECK, CORNING 5420 OR KIMBLE 27400

CAP.

STOPPER

ML 100 NO.

100

2

FLASK, VACUUM, DEWAR, CYLINORICAL, BOROSILICATE GLASS UNS IL VERED AND UNEVACUATED, WITH TUBULATION FOR UNSIL VERED ANO UNEVACUATED,

CAP. ML

CAP.

FLASK, VACUUM, DEWAR, CYLINORICAL, GOROSILICATE GLASS, COMPLETE, SILVERED AND EVACUATED, NO. 4560 , CORNING PYREX

$\begin{array}{lll}\text { CAF } & \text { ARrROX } & \text { INSIDE } \\ \text { ML } & \text { INNER TUBE } & \text { DEPTH } \\ 450 & \text { OD IN. } & \text { IN. } \\ 40 & 2 & 10\end{array}$

LASK, VACUUM, DEWAR, CYLIMORICAL, WI DE MOUTH,

GLASS. SILVERED AND EVACUATED. WITH METAL CASE,

WITHOUT CAP ANO STOPPER, 10 O2 CAP.. TYPE 7063

AMERICAN THERMOS BOTTLE

FLASK, VACUUM, OEWAR, CYLINORICAL: BOROSILICATE GLASS SILVEREO AND EVACUATED. WITH METAL SUPPORT, AMERICAN THERMOS BOTTLE

$\begin{array}{llll}\text { CAP. CC } & \text { OVERALL SILE IN. } & \text { MFR NO. } \\ 265 & 215 / 16 \times 57 / 8 & 8600 \\ 665 & 31 / 4 \times 91 / 4 & 8640 \\ 1000 & 33 / 8 \times 133 / 8 & 8645 \\ 1900 & 57 / 8 \times 10 & 8621\end{array}$

MFR NO.

$1900 \quad 5718 \times 10$

8621

$\begin{array}{lllll}\text { EA } & 2.75 & 3 & 15-090-0540 & 36 \\ \text { EA } & 2.56 & 3 & 15-090-0545 & 36 \\ \text { EA } & 3.99 & 3 & 15-090-0550 & 36\end{array}$

36

6

$\begin{array}{rrrrr}E A & 1.17 & 3 & 15-090-1690 & 36 \\ \text { EA } & 2.23 & 23 & 15-090-1745 & 36\end{array}$

EA $6.31 \quad 3.15-090-7105 \quad 36$

EA $21.04 \quad 3 \quad 15-090-7209 \quad 36$

EA $\quad 3.01 \quad 1 \quad 15-090-7254 \quad 36$

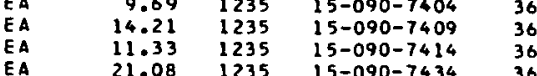


Attachment B

FLASK, VACUU,. DEMAR, FLASK SHAPE, BOROSILICATE GLASS SILVERED AND EVACUATED. WITH METAL SUPPORT. AMERICAM TMERMOS BOTTLE

$\begin{array}{ll}\text { CAP. ML } & \text { MFR } \\ 750 & 8692 \\ 1600 & 6694 \\ 4200 & 8696\end{array}$

FLASK, VACUUM, DEWAR, MIOE MOUTH, BOROSILICATE GLASS, FR No. SILVREO ANO EVACUATEO. WITH ALUMINUM METAL STAND. 2950 ML CAP.. 120 MM ID. 150 MM OD, 300 MM DEPTH. ND. M-20310, M S MARTIN, OR NO. 30020. POPE SCIENTIFIC. INC

FLASK, VACUUM, THERMOS, PYREX GLASS, SILVERED WITH ASBESTOS SEPARATION PAOS BETHEEN YALLS ANO ALUHINUM BASE, AMERICAN THERMOS BOTTLE CAP. CC 845
4300

MFR NO. 6677

FLASK, VOLUMETRIC, EXAX BLUE LINE, RETESTED, MITH SIT STOPPER 9, 10 ML CAP.. CORNING S641-KIMBLE 28015

FLASK, VOLUMETRIC, MORMAX, PREC IS ICN, GR DOUATED, WITH SIT STOPPER. CORNING 5680 OR XIMELE 28017

$\begin{array}{lll}\text { ITH SST STOPPER. CORNING } & 5680 \text { OR KIMBLE } \\ \text { CAP. } & \text { TOL } & \text { S } \\ \text { ML } & 0.03 & 9 \\ 25 & 0.05 & 9 \\ 50 & 0.08 & 1 \\ 100 & 0.08 & 1 \\ 200 & 0.10 & 1 \\ 250 & 0.12 & 1 \\ 500 & 0.15 & 2 \\ 1000 & 0.30 & 1 \\ 2000 & 0.50 & \end{array}$

STOPPER
NO.
9
9
13
13
16
19
19
27

FLASK, VOLUMETRIC, PYREX GLASS, WITH S/T STOPPER, CLASS A CORNING 5000 OR KIMBLE 20014 CAP.
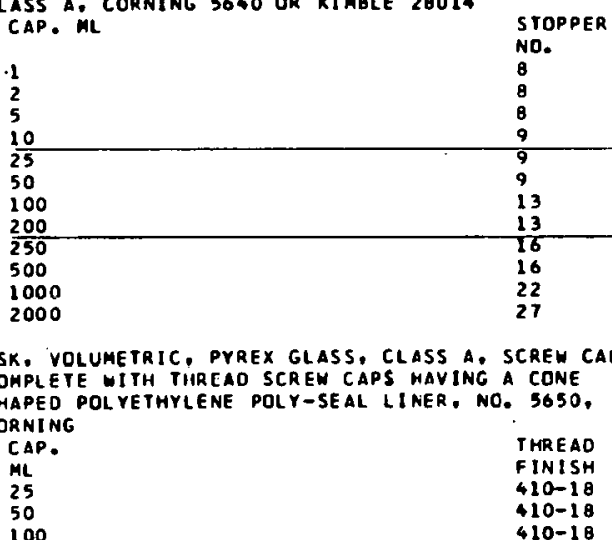

FLASK, VULUMEIKIL, PIREX; SAYOCLT, VISCOSIAETER, HL CAP.. CORNING 5860 OR KIMBLE 28126

FORCEPS, GALANCE WEIGHT. PLASTIC TIPPEO, CURYEO. MATHE SON $3505-20$

FORCEPS, BOTTLE, STAINLESS STEEL, $250 \mathrm{MM} \mathrm{LGTH,} \mathrm{NO.}$ S-35277, SARGENT, OR FSCO 10-316B

FORCEPS, COVER glass, MITH Bent ENDS, Chrome Plated, STEEL. 1/2 IN. L. SARGENT S-35195, VMR 30015-007

FORCEPS, DISSECTING. FINE CURVEO PCINTS. NICKEL PLATED FURGEU SIEEL. IIS MM LOTH: HO. 19520-10. MATME SON. SCIENT. PROD 0256

FORCEPS, DISSECTING, FINE STRAIGHT POINTS, NICKEL PLATED FORGED STEEL. 11 , mM LLIT. iNO. 19300-10. MATHESON

FORCEPS, OISSECTING, MEAVY STRAIGHT BROAC POINTS, NICKEL PLATED STEEL, BROAD SERRATICNS CN HANOLE. FINE SERRATIONS ON TIPS, $125 \mathrm{MH}$ LGTH, SARGENT S-26515 OR VWR 25722-003

FORCEPS, DISSECTING. MEOIUM CURYED POINTS. NICKEL PLATED STEEL. HIGHLY POLISHED, WITH INSIDE OF POINIS FILE CUT, 115 MH LGTH. NO. 8-882, FISHER OR PRFISER 12-2510 OR MATHE SON 19522-10

PRICE STK PT CATALOG NO BUYER

UNIT

EA

$\begin{array}{lr}37.79 & 3 \\ 46.11 & 123\end{array} \cdot \begin{aligned} & 15-090-7604 \\ & 15-090-7614\end{aligned}$

$\begin{array}{lll}4.38 & 123 & 15-090-7614 \\ & & 15-090-7624\end{array}$

36

36

EA $\quad 48.62 \quad 2 \quad 15-090-7704 \quad 36$

EA $\quad \begin{array}{lllll}11.65 & 1 & 15-090-7904 & 36 \\ & 27.51 & 123 & 15-090-7924 & 36\end{array}$

EA $2.46 \quad 3 \quad 15-090-8204 \quad 36$ 
Attachment B

UNIT PRICE STK PT CATALOG NO BUYER

FORCEPS, DISSECT ING, STUOENT, FINE STRAIGHT POINTS, NICKEL PLATEO STEEL. WITHOUT SERRATIONS ON TIPS AND HANDLE, 120 MM L $25 T$, N

\begin{tabular}{|c|c|c|c|c|}
\hline EA & .00 & 23 & $15-093-2270$ & 36 \\
\hline & & & . & \\
\hline EA & 2.11 & 123 & $15-093-2705$ & 36 \\
\hline EA & 3.68 & 1 & $15-093-2805$ & 36 \\
\hline $\begin{array}{l}\text { EA } \\
\text { EA } \\
\text { EA } \\
\text { EA }\end{array}$ & $\begin{array}{r}.20 \\
.00 \\
1.04 \\
2.07\end{array}$ & $\begin{array}{c}12 \\
2 \\
125 \\
23\end{array}$ & $\begin{array}{l}15-093-2850 \\
15-093-2855 \\
15-093-2860 \\
15-093-2865\end{array}$ & $\begin{array}{l}36 \\
36 \\
36 \\
36\end{array}$ \\
\hline EA & 37.20 & 23 & $15-093-2915$ & 36 \\
\hline$E A$ & .21 & 23 & $15-093-2935$ & 36 \\
\hline EA & .52 & 123 & $15-093-2965$ & 36 \\
\hline & & . & & \\
\hline EA & .20 & 3 & $1 b-096-0250$ & 36 \\
\hline EA & 2.45 & 3 & $15-096-0306$ & 36 \\
\hline
\end{tabular}
FUNNEL, BUCHNER, POLYETHYLENE, 70 MM OIA, FOR FILTER PAPER, MATHESON 25237-10

ORCEPS, KELLEY hEMOSTAT, CHROME PLATEO, STRAIGHT, BOX LOCK. $51 / 2$ IN. LGTH. NO. 5026. KAY SURGICAL FSCO 8-907 OR VWR 258BI-009

FORCEPS, MICRO, SHISS STYLE, WATCMMAKERS TYPE, FINE POINTS, SS, 4 1/8 IN. LGTH, NO. A-1905. CLAY ADAMS. REPL PT

FORCEPS, SPECIMEN JAR. NICKEL PLATEO STEEL. TIPS ROUNDEO AND FILE CUT, NO. 10-316, FISHER

150 IMATHESON $19545-101$

175 IMATHESON $19545-201$

LAPINE $10951-02$, PREISER 12-7542-28

LAPINE 10951-03, PREISEN 12-7542-30

FORCEPS, STRAIGHT, STAINLESS STEEL, PLATINUM TIPPED, 127 MM LGIH, FISHER 10-285 OR SARGENT S-35185 ENGELHARD 507 OR VHR 30049-009

FORCEPS. TISSUE, STRAIGHT MOUSE TOOTH IIPS, NICKEL PLATEO FORGEO STEEL. 115 MM LGTH. NO. 19560-10. MATHESON OR VHR 30010-003

FORCEPS, UTILITY, STAINLESS STEEL. ALL PURPOSE, 140 MM LGTH, VWR $25719-066$

FUNNEL, ANALYTICAL, POLYPROPYLENE, FOR ORGANIC SOLVENTS AND STRONG ACIOS ANO BASES, 65 MM IO, 65 MM STEM LGTH, FOR 110 MM FILTER PAPER, 36 PER CASE. ILL 14192. MATHF, SNN 25099-25

FUNNEL, BUCHNER, PORCELAIN, WITH FIXED PERFORATEO PLATE, GLALEO INSIDE AND OUTSIOE WITH EXCEPTION OF RIA

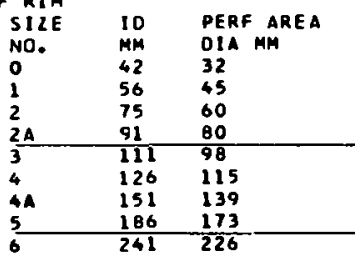

OVERALL
HT MM
69
101
143
160
195
202
221
270

COOR 5

ND.

49002

005

0006

9010

$5-096-0306$

FUNNEL, BUCHNER, PYREX GLA55, CAP. $450 \mathrm{ML}$,

POROSITY UITH ACE FIBER GLASS FILTER OISC, 9O NO. $7106-040$ OCE GLASS OR LAGLASS NOO LG-7OBO-S

FUNNEL, BUCHNER, PYREX GLASS, WITH FRITTEO DISC, CORNING $36060^{\circ}$ OR KIMBLE 28400

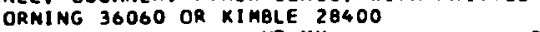

\begin{tabular}{|c|c|c|c|c|c|c|c|}
\hline $\begin{array}{l}\text { CAP. } \\
\text { ML }\end{array}$ & POROSITY & $\begin{array}{l}\text { HT MM } \\
\text { ABOVE DISC }\end{array}$ & $\begin{array}{l}\text { DISC } \\
\text { OIA MM }\end{array}$ & & & & \\
\hline 15 & MEOIUH & & & EA & 4.42 & 3 & $15-096-0755$ \\
\hline 30 & COARSE & 45. & 30 & EA & 4.98 & 23 & 15-096-0Rก5 \\
\hline 30 & KEDIUH & 45 & 30 & EA & 4.80 & 3 & $15-096-0808$ \\
\hline 30 & FINE & 45 & 30 & EA & 4.09 & 23 & $15-096-0811$ \\
\hline 60 & COARSE & 50 & 40 & EA & 5.82 & 5 & $15-096-0 \mathrm{1} 14$ \\
\hline 60 & MEDIUM & 50 & 40 & EA & 5.50 & 123 & $15-096-0817$ \\
\hline 60 & FI NE & 50 & 40 & EA & 5.36 & 3 & $15-096-0820$ \\
\hline 150 & COARSE & so & 60 & EA & 9.00 & 23 & $15-096-0823$ \\
\hline 150 & MEOIUM & 50 & 60 & EA & 8.99 & 23 & $15-096-0826$ \\
\hline 150 & FINE & so & 60 & EA & 8.11 & 23 & $15-096-0829$ \\
\hline 350 & COARSE & 75 & 80 & EA & 11.22 & 23 & $15-096-0832$ \\
\hline 350 & MEOIUM & 15 & 80 & EA & 12.53 & 23 & $15-096=0835$ \\
\hline 350 & FINE & 75 & 80 & EA & 11.22 & 3 & $15-096-0838$ \\
\hline 600 & COARSE & 90 & 90 & EA & 13.45 & 1 & $15-096-0841$ \\
\hline 600 & MEOIUM & 90 & 90 & EA & 13.45 & 12 & $15-096-0844$ \\
\hline 600 & FINE & 90 & 90 & EA & 46 & 1 & $15-096-0847$ \\
\hline
\end{tabular}

$\begin{array}{lrrrr}\text { EA } & 2.49 & 23 & 15-096-0506 & 36 \\ \text { EA } & 3.02 & 123 & 15-096-0509 & 36 \\ \text { EA } & 5.43 & 23 & 15-096-0512 & 36 \\ \text { EA } & 6.49 & 23 & 15-096-0515 & 36 \\ \text { EA } & 7.16 & 3 & 15-096-0518 & 36 \\ \text { EA } & 12.82 & 3 & 15-096-0521 & 36 \\ \text { EA } & 14.87 & 3 & 15-096-0524 & 36 \\ \text { EA } & 18.08 & 2 & 15-096-0527 & 36 \\ \text { EA } & 33.93 & 23 & 15-096-0530 & 36\end{array}$

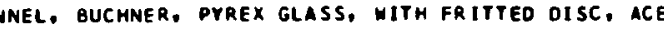
GLASS

$\begin{array}{lllll}\text { CAP. POROSITY } & \text { HT MM } & \text { OISC } & \\ \text { ML } & \text { MICRONS } & \text { ABOVE OISC } & \text { OIA MM ACE NO. } \\ 2500 & \text { IAI } 145-175 & 150 & 150 & 7186-82\end{array}$

FUNMEL, BURETTE, GLASS, 40 MM OIA, 40 MM STEM LGTH, PREISER 10-8550 OR MATHESON 6430-10

FUNNEL, FLUTEO, BOROSILICATE GLASS, ACCURATE 60 OEG LONG STEM. LGTH OF STEM 150 MM. NO. 6160, CORNING DIA

EA $21.50 \quad 3 \quad 15-096-0705$ 
Attachment B

UNIT PRICE STK PT CATALOG NO BUYER

FUNNEL, FLUTED, BOROSILICATE GLASS, ACCURATE 60 OEG, LONG SIEM, LGTH OF STEM $150 \mathrm{MM}$. NO. 6160. CORNING ONG STEMO 65 100

-CONTINUEONel. Fluteo, borosilitcate glass. accurate 60 deg. NO. 6180. CORNING PYREX DIA MM 50
75

SHORT STEM LGTH MM 65
75

FUNNEL, HIRSCH, PORCELAIN, MITM fixed PERforated

PLATE

$\begin{array}{llll}\text { SILE } & \text { TAP DIA } & \text { PLATE } & \text { COORS } \\ \text { NO. } & \text { MM } & \text { DIA MM } & \text { NO. } \\ 0000 & 55 & 16 & 51002 \\ 000 & 50 & 31 & 51003\end{array}$

FUNNEL, MICRO FILTER TUBE, BOROSILICATE GLASS, WITH FRITTEO DISC, 3 MM TOP OIA, CORNING 36290 OR KIMBLE 28450 POROSITY

MEOIUM

FUnNel, plain. GLASS, KIMBLE 58, SHORT STEM, NO. 28950. KIMBLE

STEM LGTH MM DIA MM

60

45

FUNAEL, PLAIN, GLASS, LONG STEM, SIDE ANGLE 00 DEG, ISO MM STEM LGTH, NO. 28900 , KIMELE

DIA MM

55

65
100

FUNNEL, PLAIN, glass, SHORT STEM, SIDE ANGLE 60 OEg, NO. 29000. KI MBLE

DIA

$\operatorname{MM}_{25}$

250

STEM LGTH

40

150

FUNNEL, PLAIN, GLASS, 60 DEG STEM LGTH IN. DIA IN. CORNING NO. KIMBLE NO. $\begin{array}{cccc}4 & 5 & 6120 & 28960 \\ 4 & 6 & 6120 & 28960\end{array}$ $\begin{array}{llll}4 & 6 & 6120 & 28960 \\ 6 & 6 & 6100, & 28900\end{array}$

FUnNel, PLAIN, gLASS, accurate 60 DEg, LONG STEM, 150 MM STEM LGTH. NO. BI40. CORNING OR KIMBLE 28900 DIA MM

50

100

FUNMEL, POLYETHYLENE, IN. DIA, SCHAAR 14228 F5CO 10-271C OR MATHESON 25097-20

FUNNEL, POLYETHYLENE, 6 IN. OIA, VMR 30251-101

FUNNEL, POLYETHYLENE, B IN. OIA, MILL 14228, MATHESON 23097-40, OR SARGENT S-35431F

FUNAEL. PONDER, GLASS, MITH SHORT WIDE STEM AND FIRE DOL ISHED TOP AND STEM, 60 MM DIA, IS MM STEM DIA. 25 MM STEM LGTH. NO. 29020, KIMBLE

FUNNEL, RIBBED, MOLOED GLASS, SHORT STEM, 32 OL CAP.. 7 I/4 IN. OIA. SARGENT S-35385-E OR FSCO 10-381-D

FUNNEL. SEPARATORY, CYL SHAPE, DPEN TOP ANO LONG STEM WITH SIT STOPCOCK, CORNING 6420 OR KIMBLE 29026 CAP. ML

125

FUNMEL, SEPARATORY, CYLINORICAL, OPEN TOP, CAP, 200 ML. SIT STOPCOCK MO. 3. NO. LG-8320-S. LABGLISS

FUMMEL. SEPARATORY, CYL SHAPE, BOROSILICATE CLASS, OPEN TOP, CAP. $250 \mathrm{ML}$. WITH NO. 4 STT STOPCOCR. CORNING O420
CONTINUE-

$\begin{array}{lllll}\text { EA } & .68 & 13 & 15-096-1510 & 36 \\ \text { EA } & .93 & 1235 & 15-096-1515 & 36 \\ E A & .97 & 235 & 15-096-1520 & 36\end{array}$

$\begin{array}{lllll}\text { EA } & .58 & 1 & 15-096-1560 & 36 \\ \text { EA } & 1.00 & 1 & 15-096-1565 & 36\end{array}$

$\begin{array}{lllll}E A & 2.06 & 23 & 15-096-1805 & 36 \\ \text { EA } & 2.10 & 3 & 15-096-1007 & 36\end{array}$

$\begin{array}{lllll}\text { EA } & 3.69 & 3 & 15-096-2206 & 36 \\ \text { EA } & 3.63 & 3 & 15-096-2207\end{array}$

$\begin{array}{lllll}\text { EA } & .42 & 23 & 15-096-2505 & 36 \\ & .60 & 23 & 15-096-2515 & 36\end{array}$

$\begin{array}{rrrrr}\text { EA } & .57 & 23 & 15-096-2605 & 36 \\ \text { EA } & .68 & 3 & 15-096-2610 & 36 \\ \text { EA } & 1.64 & 3 & 15-096-2615 & 36\end{array}$

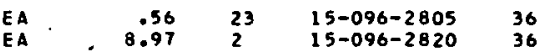

$\begin{array}{lllll}\text { EA } & 2.01 & 235 & 15-096-2880 & 36 \\ \text { EA } & 1.84 & 12 & 15-096-2885 & 36 \\ \text { EA } & 2.64 & 1 & 15-096-2895 & 36\end{array}$

$\begin{array}{llrrr}\text { EA } & .71 & 3 & 15-096-3205 & 36 \\ \text { EA } & .74 & 3 & 15-096-3210 & 36 \\ \text { EA } & .92 & 23 & 15-096-3215 & 36\end{array}$

EA $\quad 00 \quad 12 \quad 15-096-3410 \quad 36$

EA $\quad .37 \quad 23 \quad 15-096-3415 \quad 36$

EA $\quad .00 \quad 2 \quad 15-096-3420 \quad 36$

EA $\quad .70 \quad 123 \quad 15-096-3605 \quad 36$

EA $\quad .00 \quad 2 \quad 15-099-0506 \quad 36$

$\begin{array}{lllll}\text { EA } & 6.22 & 3 & 15-099-1006 & 36 \\ \text { EA } & 6.85 & 3 & 15-099-1016 & 36\end{array}$

EA $6.83 \quad 3 \quad 15-099-1043 \cdot 36$

EA $\quad 7.70 \quad 3 \quad 15-099-1059 \quad 36$ 
FUNAEL, SEPARATORY, CYL SHAPE, MITH S/T STOPCOCK AND $\begin{array}{lll}\text { TOPPER, CORNING } & 380 \text { OR KIMBLE } 29033 & \\ \text { CAD. ML } & \text { STOPCOCK NO. } & \text { STOPPER NO. } \\ 250 & 2 & 16 \\ 1000 & 4 & 22\end{array}$ UNNEL, SEPARATORY, GLOBE SHAPE WITH S/T STOPCOCK ANO STOPPER, CORNING 6340 OR KIMELE 29043

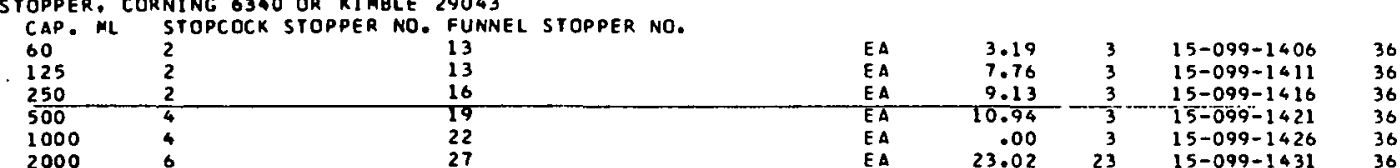

FUNNEL, SEPARATORY, PEAR SHAPE, SOUIBB, BOROSILICATE GLASS. WITH GROUNO GLASS STOPPER, 30 ML CAP.. NO. LG-8420, LABGLASS

EA $\quad 4.40 \quad 23 \quad 15-099-1506 \quad 36$

FUNNEL, SEPARATORY, PEAR SHAPE, SOUIBB, WITH SIT

STOPCOCK AND STOPPER, CORNING 6400 OR KIMBLE 29048

CAP.

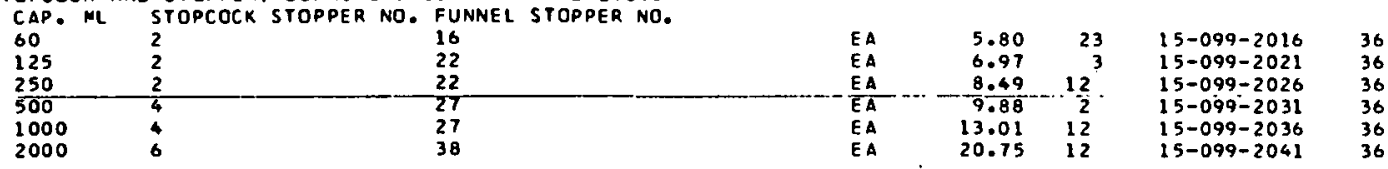

FUNHEL. SEPARATORY, CENTRIFUGAL, BCROSILICATE GLASS, CYLINDRICAL SHAPE, MITH TEFLCN STOPCOCK AND SCREY CYLINDRICAL SHAPE, WITH TEFLCN

CAP TOP

CAP.

30

60

250

FUNNEL, SEPARATORY, BOROSILICATE GLASS, CAP, $125 \mathrm{ML}$. TEFLON VALVE, TYPE K-4535, KONTES

FUNNEL, SEPARATORY, SOUIBB TYPE, CAP. $125 \mathrm{ML}$, WITH SIRAIGHT BORE GYCO STOPCOCK. NO. JF-BG74, SGA

GAS, COMPRESSEO, EFFA DUSTER REFILL. MICROSCOPICALLY CLEAN, 15 OL CAN, NO. 1168, ERNEST F FULLAM, INC

GAUGe. MCLEOD, Borosillicate GLASS, RaNGE O-5. I MPROVEO FROM INTER-JOINT, COMPLETE. CAP. OF FLASK $500 \mathrm{ML}$. S/T JOINT NO. 24/40, NO. JV-2490, SGA

GLASS, COVER, SOUARE, NO, I THICKNESS, SIZE 22 MM, TEN 1 OZ BOXES PER PKG. NO. 2865 . CORNING, NO SUB

GI,ASS, COVER, RECTANGULAR, NO. I THICKNESS, TEN I OL BOXES PER PKG. NO. 2935. CORNING. NO SUB

5 ILE $M M$

$22 \times 30$

$22 \times 40$ INO SUB AT $Y-121$

$\frac{22 \times 50}{22 \times 60}$

$24 \times 50$

ASS, COVER, HICRO, NON-CORROSIVE, ROUND, NO. 2.

$18 \mathrm{MM}$ DIA. THICKNESS RANGE 0.17 TO $0.25 \mathrm{MM}, 1 / 202$ 18 MM DIA, THICKNESS RAM
BOX. NO. 2915 . CORNING

GLASS. COVER, MICRO, NON-CORROSIVE, 1 OZ BOX, A H

THOMAS

HAPE SILE MM MFR NO.

ROUND 22 (OIA) 7022 RED LABEL NO.

SOUARE 18 TO2O REO LABEL NO. I

SOUARE 227020 REO LABEL NO. 1 ,

SOUARE 25 TO2O REO LABEL NO. 1

7020 RED LABEL NO
OR VMR $48366-089$

GLAZE. LABEL. 4 OZ BTL. FSCO 11-880, OR VMR 36419-701

GRADUATE, BEAKER, BOROSILICATE GLASS, DOUBLE SPOUT, DOUBLE SCALE, NO. G4BD, CORNING PYREX

CAP. ML

500

graouate, pharmaceutical, glass, blue line exax, reTESTEO. SIMGLE METRIC SCALE, No. 60340, KIMBLE

CAP. ML

5

\begin{tabular}{|c|c|c|c|}
\hline $\begin{array}{l}\text { EA } \\
\text { EA } \\
\text { EA } \\
\text { EA }\end{array}$ & $\begin{array}{l}7.78 \\
7.78 \\
7.75 \\
0.99\end{array}$ & $\begin{array}{r}3 \\
3 \\
3 \\
23\end{array}$ & $\begin{array}{l}15-099-2200 \\
15-099-2210 \\
15-099-2220 \\
15-099-2230\end{array}$ \\
\hline EA & 7.78 & 3 & $15-099-2306$ \\
\hline EA & 8.95 & 1 & $15-099-3000$ \\
\hline CAN & 1.34 & 2 & $15-102-0300$ \\
\hline$E A$ & 100.74 & 3 & $15-106-0927$ \\
\hline $80 x$ & 2.41 & 2 & $15-106-1395$ \\
\hline $\begin{array}{l}B O x \\
B O x \\
B O x \\
B O x \\
B O x \\
B O x\end{array}$ & $\begin{array}{l}2.77 \\
2.24 \\
2.41 \\
2.40 \\
2.40 \\
2.40\end{array}$ & $\begin{array}{l}2 \\
23 \\
2 \\
2 \\
2 \\
2\end{array}$ & $\begin{array}{l}15-106-1502 \\
15-106-1507 \\
15-106-1512 \\
15-106-1515 \\
15-106-1519 \\
15-106-1524\end{array}$ \\
\hline Box & 3.64 & 3 & $15-106-1557$ \\
\hline $\begin{array}{l}80 x \\
80 x\end{array}$ & $\begin{array}{l}5.78 \\
1.18\end{array}$ & $\begin{array}{r}23 \\
3\end{array}$ & $\begin{array}{l}15-106-1577 \\
15-106-1587\end{array}$ \\
\hline Box & 1.16 & 2 & $15-106-1590$ \\
\hline Box & .00 & 3 & $15-106-1593$ \\
\hline BTL & .00 & 1 & $15-106-2807$ \\
\hline EA & 5.28 & 1 & $15-106-4217$ \\
\hline $\begin{array}{l}E A \\
E A\end{array}$ & $\begin{array}{l}2.65 \\
4.17\end{array}$ & $\begin{array}{l}3 \\
3\end{array}$ & $\begin{array}{l}15-106-4707 \\
15-106-4710\end{array}$ \\
\hline
\end{tabular}


Attachment B

UNTT PRICE STK PT CATALOG NO BUYER

Pipette, micro, transfer tYpe, pyrex glass, selfFILIING, MICROCHEMICAL SPECIALTIES CO CAP. LAMBDA

1
10

SER!ES NO.

$M C A-1$

MCA-2

MCA-7

petTe, pasteur capillary, disposable, overall lgth

$53 / 4$ IN.. CAPILLARY $21 / 4$ IN. LGTH $\times 1 / 2 \mathrm{MM} 10$

SODY 312 IN LGTH $X 7$ MM OD. $21 / 2$ GROSS PER

BOX. MATHESON 55698-00 OR VHR 14672-029

BOX

2.98

$2.16 \quad 3 \quad 15-169-4740$ $1.70 \quad 3 \quad 15-169-4750$ $\begin{array}{lll}1.70 & 3 & 15-169-4770\end{array}$ $5-169-4785 \quad 36$

pipette, serological, blue line exax, retested, no.

37034, KIMBLE
CAP. ML SUB OIV ML

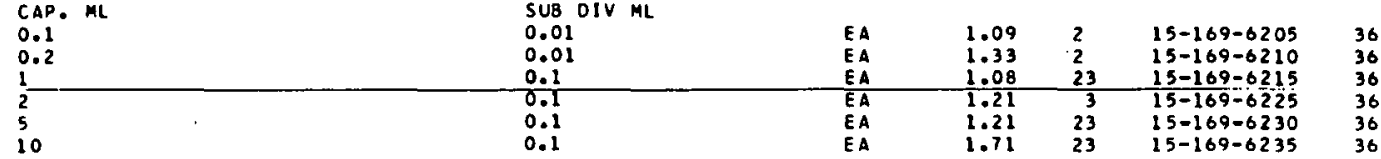

PIPETTE, SEROLOGICAL, INTERVAL, CORNING 7085 OR

KIMBIE 37033 COCAL. INTERVAL, CORNING TOBS OR

(

5

$\begin{array}{ll}\text { COLOR CODEO } & \text { GRAD ML } \\ \text { YELLOH } & 11100 \\ \text { BLUE } & 1 / 10 \\ \text { ORANGE } & 1110\end{array}$

$\begin{array}{lllll}\text { EA } & 1.00 & 2 & 15-169-6405 & 36 \\ \text { EA } & 1.00 & 2 & 15-169-6415 & 36 \\ \text { EA } & 1.14 & 2 & 15-169-6420 & 36\end{array}$

pipette, serological, polypropylene, all Sizes 0.10

ML SUBOIV.. NALGE 3610, OYNALAB 1100

CAP. ML

1.0 MATHESON 55\%53-20

2.0 MATHESON 55653-30

5.0 MATHESON $55653=40$
10.0 MATHESON $55653-45$

PIPETte, transfer, disposable, ethylux plastic SPITZER tUBe. Westlake PLASTICS Co

PIPETTE, ULTRA-MICRO, LAMBDA, BOROSILICATE GLASS, WITH GROUNO TIPS. ETCHED HAIRLINE CALIBRATION MARKING, SCIENTIFIC SUPPLIES CO

CAP. LAMBOA

?

PIPETte, voluaetric or transfer, Class a kimax. No. 37010. KIMBLE

IAP ML

TOL ML

PLUS/MINUS

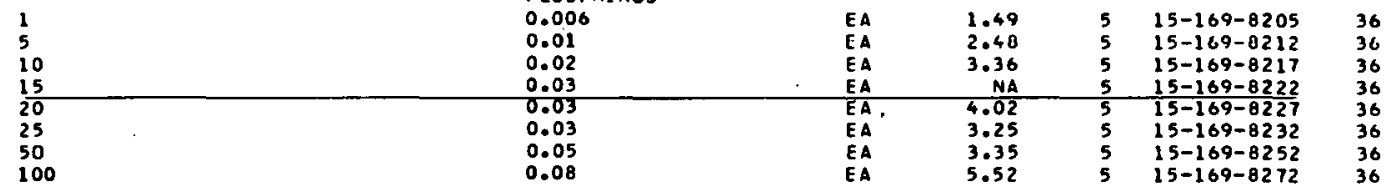

PIPETTE, VOLUMETRIC OR TRANSFER, ClASS A PYREX OR

I UOY. NO. 71 BO CORRING OR NO. 37004, KIMBLF

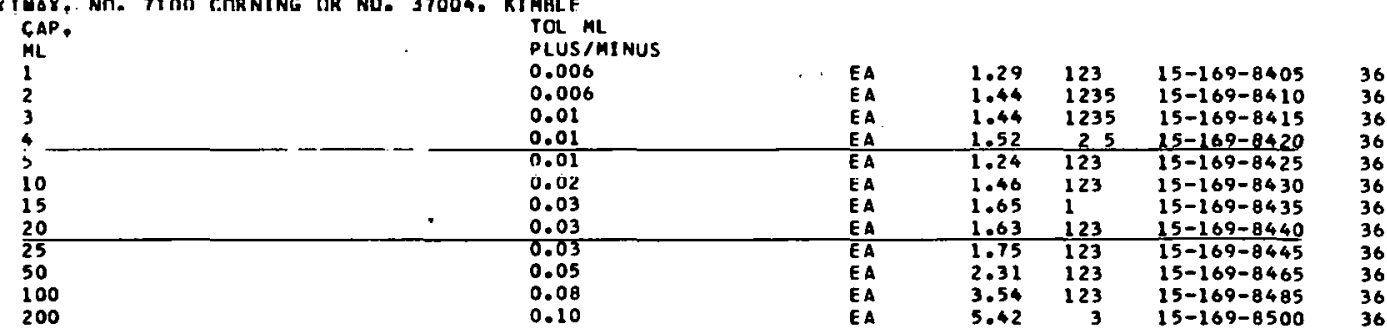

PIPETTE, VOLUMETRIC OR TRANSFER, BLUE LINE EXAX, RETESTED, WITH LONG OELIVERY STEM. ML CAPAO NO. 7102, CORNING OR XIMBLE 37000

$\begin{array}{lrrrr}\text { FA } & 1.97 & 3 & 15-169-6405 & 36 \\ \text { EA } & 1.85 & 3 & 15-169-6610 & 36 \\ \text { EA } & 2.15 & 3 & 15-169-6615 & 36 \\ \text { EA } & 2.32 & 3 & 15-169-6620 & 36 \\ & & & & \\ \text { EA } & 1.23 & 3 & 15-169-6835 & 36 \\ & & \ldots & & \\ & & & & \\ \text { EA } & & & & \\ \text { EA } & 2.15 & 3 & 15-169-7505 & 36 \\ \text { EA } & .63 & 3 & 15-169-7510 & 36 \\ & .87 & 3 & 15-169-7515 & 36\end{array}$

PIPETTE FILLER IPIPETTORI. CAULIFIELO, CONSISTS OF RUBBER BULB WITH ENCLOSED VALVE ANO VALVE CONTROL ON TOP. WILL DRAH UP AND DISCHARGE TO LESS THAN 1/100 ML. WILL 22103 OR MATHESNM 55900-10

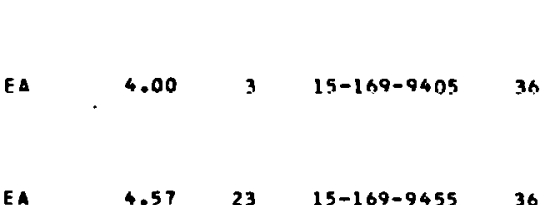

PROPIPETTE, AUTOMATIC, CONSISTS OF CONTROL OF SOLUTION IN PIPETTE. MATHESON $55900-10$ FSCO 13-681-50 
Attachment B

PIPETTOR, 500 ML FLASK, PREISER

SCIENTIFIC CORP

DEL IVERY VOLUME ML

DEL I VERY VOLUME AL

10 CMS $251-777$
15 ILL 22093

plate, oesiccator, porcelath, glazeo one side, with THREE

$\begin{array}{cccc}\text { PHREE SMALL } & \text { FEET AND } 30 \mathrm{MM} \text { DIA HOLES, } 6 \text { MH THK } \\ \text { SIZE NO. } & \text { OIA MH } & \text { NO. HOLES } & \text { COORS NO. } \\ 3 & 142 & 5 & 57004 \\ 4 & 190 & 7 & 57006 \\ 5 & 230 & 8 & 57007\end{array}$

plate, desiccator, porcelain, glazed one side, with FEET. HOLE OIA 30 MH, SIZE NO. 3A, OIA $148 \mathrm{MH}$. 6 MM THK. 7 HOLES, COORS 57005

plate. oesiccator. porcelain, glazeo one side,

WITHOUT FEET, 5 MA PERFORATIONS. LARGE HOLE IN CENTER

$\begin{array}{clll}\text { CENTER } & & & \\ \text { SILE } & \text { THK } & \text { PLATE } & \text { COORS } \\ \text { NO. } & \text { IN. } & \text { DIA MM } & \text { NO. } \\ 5 & 6 & 230 & 60003 \\ 3 & 5 & 140 & 60001\end{array}$

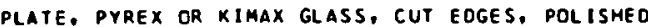
FACES. NO. 7740 CORNING OR NO. KG-33 KIMBLE, OR APPROVED EQUAL

SILE IN.

$3 / 8 \times 15 \times 30$

$1 / 2 \times 15 \times 30$

$3 / 4 \times 15 \times 30$

plate, pyrex glass, flat, eoges cut SILE IN.

SILE IN $\times$ is $\times 30$

$3 / 16 \times 15 \times 30$

$114 \times 15 \times 30$

PLATE, PYREX GLASS, FLAT, GROUNO EOGES

$S I Z E$ IN.

$1 / 16 \times 61 / 2 \times 61 / 2$

$1 / 8 \times 61 / 2 \times 6112$

$3116 \times 6 \quad 112 \times 6 \times 1 / 2$

PLATE, PYREX OR FUREX, FLAT, WATER CLEAR FOR $\checkmark$ IS IBILITY. CUT EDGES. SILE $1 / 2 \times 24 \times 24$ IN.

PLATE, OUARTZ, POLISHED

SILE IN.

$1 / 16 \times 6 \times 6$

$\begin{array}{lllll}1,0 & \times & 8 & \times & 0 \\ 114 & \times & 6 & \times & 6\end{array}$

$3 / 8 \times 6 \times 6$ 'CLEAR, FUSED, POLISHED FACES AND GRUUNU EUGES, GE TYME 101 OR

PLATE, SPOT, COLOR REACTION, PORCELAIN, CIA OF DEPRESSIONS $21 \mathrm{MM}$, OEPTH OF DEPRESSIONS $7.5 \mathrm{mM}$, 13 MM THK. T.OOR

$\begin{array}{llllll} & \text { LIZE } & \text { LGIH } & \text { NIOTH } & \text { NO. } & \text { MFR } \\ \text { GLAZED } & \text { NO. } & \text { MM } & \text { MM } & \text { DEPRESSIONS NO. } \\ \text { BLACK } & 1 & 118 & 91 & 12 & 55105 \\ \text { WHITF } & 000 & 109 & 89 & 12 & 55007\end{array}$

plug, Closure, disposable, polyurethane foam, autoCLAVABLE, FOR LAB GLASSWARE, SC IENTIFIC PROOUCTS

TO FIT OPENINGS MM NO. PER PKG MFR NO. 6 TO 15 16 10 22

$T-1383$

$\begin{array}{lll}28 & 200 & T-1385\end{array}$

POL ICEMAN, RUBBER, WITH ENOS SEALEO FLAT AND CUT AT ANGLE, NO. 14-105, FISHER, NO. 13-9310. PREISER

FOR ROD IN

$1 / 8$ SARGENT $5-732258$, MATHESON 57500-05

3116 SARGENT S-73225A. MATHESON 57500-10

POLICEMAN, RUBBER, SOFT AND THIN, WIDE FCRM, $11 \%$

IN. LGTH X 1 IN. WIOTH, ATTACHED TO $3 / 16 \times 6$ IN.

GLASS ROO. MATH. 57510-10 OR VWR 53803-003 ANO 3 PR $50060-047$

POLISH, ABRASIVE, GaMAL ALUMINA, 4 OL BTL,

FSCO A-446 OR VHR $40400-048$

\begin{tabular}{|c|c|c|c|c|}
\hline & & & & \\
\hline UNIT & PRICE & STK PT & CATALOG NO & BUYER \\
\hline $\begin{array}{l}\text { EA } \\
\text { EA } \\
\text { EA }\end{array}$ & $\begin{array}{r}12.26 \\
8.72 \\
12.82\end{array}$ & $\begin{array}{l}3 \\
3 \\
3\end{array}$ & $\begin{array}{l}15-169-9705 \\
15-169-9710 \\
15-169-9715\end{array}$ & $\begin{array}{l}36 \\
36 \\
36\end{array}$ \\
\hline $\begin{array}{l}\text { EA } \\
\text { EA } \\
\text { EA }\end{array}$ & $\begin{array}{l}4.55 \\
6.93 \\
9.32\end{array}$ & $\begin{array}{r}2 \\
23 \\
3\end{array}$ & $\begin{array}{l}15-172-1020 \\
15-172-1015 \\
15-172-1020\end{array}$ & $\begin{array}{l}36 \\
36 \\
36\end{array}$ \\
\hline EA & 4.90 & 3 & $15-172-2100$ & 36 \\
\hline & & & $=$ & \\
\hline $\begin{array}{l}\text { EA } \\
\text { EA }\end{array}$ & $\begin{array}{l}9.95 \\
5.11\end{array}$ & $2_{3}$ & $\begin{array}{l}15-172-1210 \\
15-172-1290\end{array}$ & $\begin{array}{l}36 \\
36\end{array}$ \\
\hline $\begin{array}{l}\text { EA } \\
\text { EA } \\
\text { EA } \\
\text { EA }\end{array}$ & $\begin{array}{l}55.83 \\
45.00 \\
73.90 \\
53.90\end{array}$ & $\begin{array}{l}3 \\
3 \\
3 \\
3\end{array}$ & $\begin{array}{l}15-172-1710 \\
15-172-1714 \\
15-172-1718 \\
15-172-1728\end{array}$ & $\begin{array}{l}36 \\
36 \\
36 \\
36\end{array}$ \\
\hline $\begin{array}{l}\text { EA } \\
\text { EA }\end{array}$ & $\begin{array}{l}25.79 \\
30.98 \\
28.78\end{array}$ & $\begin{array}{r}23 \\
3 \\
23\end{array}$ & $\begin{array}{l}15-172-1750 \\
15-172-1754 \\
15-172-1762\end{array}$ & $\begin{array}{l}36 \\
36 \\
36\end{array}$ \\
\hline $\begin{array}{l}\text { EA } \\
\text { EA } \\
\text { EA } \\
\text { EA }\end{array}$ & $\begin{array}{l}4.02 \\
2.90 \\
3.55 \\
3.26\end{array}$ & $\begin{array}{l}3 \\
3 \\
3 \\
3\end{array}$ & $\begin{array}{l}15-172-1770 \\
15-172-1780 \\
15-172-1790 \\
15-172-1800\end{array}$ & $\begin{array}{l}36 \\
36 \\
36 \\
36\end{array}$ \\
\hline EA & 61.86 & 2 & $15-172-1850$ & 36 \\
\hline $\begin{array}{l}\text { EA } \\
\text { EA } \\
\text { EA }\end{array}$ & $\begin{array}{l}30.78 \\
39.10 \\
44.63\end{array}$ & $\begin{array}{r}23 \\
43 \\
3\end{array}$ & $\begin{array}{l}15-172-1900 \\
19=172-1410 \\
15-172-1970\end{array}$ & $\begin{array}{l}97 \\
41 \\
97\end{array}$ \\
\hline$P C$ & NA & 3 & $15-172-1940$ & 97 \\
\hline $\begin{array}{l}E A \\
E A\end{array}$ & $\begin{array}{l}4.90 \\
2.25\end{array}$ & $\begin{array}{l}23 \\
23\end{array}$ & $\begin{array}{l}15-172-1960 \\
15-172-1980\end{array}$ & $\begin{array}{l}36 \\
36\end{array}$ \\
\hline $\begin{array}{l}\text { PKG } \\
\text { PKG } \\
\text { PKG }\end{array}$ & $\begin{array}{r}7.15 \\
11.20 \\
3.72\end{array}$ & $\begin{array}{l}2 \\
2 \\
2\end{array}$ & $\begin{array}{l}15-172-2400 \\
15-172-2410 \\
15-172-2420\end{array}$ & $\begin{array}{l}36 \\
36 \\
36\end{array}$ \\
\hline $\begin{array}{l}\text { EA } \\
\text { EA }\end{array}$ & $\begin{array}{l}.00 \\
.00\end{array}$ & $\begin{array}{l}12 \\
1235\end{array}$ & $\begin{array}{l}15-172-3200 \\
15-172-3210\end{array}$ & $\begin{array}{l}36 \\
36\end{array}$ \\
\hline EA & .10 & 23 & $15-172-3350$ & 36 \\
\hline B TL & 6.30 & 5 & $15-172-3650$ & 36 \\
\hline
\end{tabular}


Attachment B

UNIT PRICE STK PT CATALOG NO BUYER

RING, CORK, NO. 7-835, FISHER

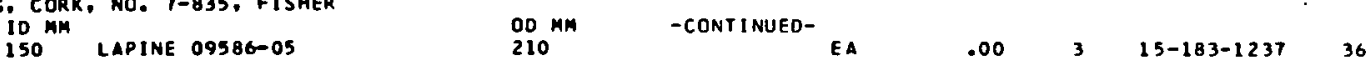

RING, EXTENSION, IRON, FISHER 14-0S5

$\begin{array}{lllll}2118 & 23 / 4 & 8 & \begin{array}{l}\text { PRE ISER 13-8655 } \\ \text { SARGENT S-73055B }\end{array} \\ 31 / 4 & 37 / 8 & 71 / 2 & \begin{array}{l}\text { MATHESCN } 57360-10 \\ \text { SARGENI S-73055C }\end{array} \\ 11 / 4 & 51 / 8 & 7 & \text { SARGENT S-730550 } \\ 5518 & 6114 & 6318 & \begin{array}{l}\text { WILL } 23226 \\ \text { SARGENT S-73055E }\end{array} \\ 513 / 16 & 611 / 16 & 63 / 4 & \text { SARGENT S-73055-X }\end{array}$

RING FORMS. BAKELITE, 3/4 IN. LONG, WITH 0.05 IN. WALL, BUEMLER CO OR APPROVEO EQUAL SIIE IN.

1

$\begin{array}{lll}1 & 1 / 4 \\ 1 & 1 / 2\end{array}$

MFR NO,

$20-8151-500$ $20-8152-500$
$20-8153-500$

RING, RASCHIG, DISTILLING COLUMN PACKING. GLASS,

SILE 6 MM $X$ O MM, 1 MM WALL. NO. $21020-03$. MATHESON KIMBLE 39100

RING, RASCHIG, BOROSILICATE GLASS, 5/8 IN, OO, MS $15-A A-B$

RING, RASHIG, BOROSILICATE GLASS, FIRE PQLISHED, ANNEALEU. TEMPERED. LENGTH I 3/4 IN. PLUS 0.035 MINUS 0.125 IN.O OD $11 / 2$ IN. PLUS 0.0 MINUS 0.0625 IN. WALL THK 0.219 IN. PLUS 0.060 MINUS 0.0 IN..
324 RINGS PER CONTAINER, PER UCCND SPEC Y-MS-169

RING, RASCHIG, KNIGHT-MARE, SILE I IN. OD $\times 1$ IN. LONG. MAUR ICE A KNIGHT

RING. SUPPORT, IRON, WITH CLAMP, NC, 18005, CENCO

ID $I N$.

1 3/8 MATHESON 57350-05

$21 / 2$ SARGENT $S-730458$

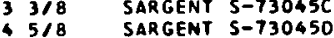

OD IN.

ING, TRUNNION, SILE $50 \mathrm{ML}$, NO, 325. INTERNATIONAL EOUIPMENT CO OR LAPINE EO0325

RING. TRUNNION, SIZE $100 \mathrm{ML}$, NO, 350, INTERMATIONAL EOUIPMENT CO

RING. TRUNNION. FOR CENTRIFUGE TYPE C ANO S-B, SIZE $10 \mathrm{ML}$ AND $15 \mathrm{ML}$. FOR METAL TUBES. IEC 310 , SCIENT. PRODUCTS C2700 OR LAPINE E00310

ROO. CARBON, NOT GRAPHITE, HIGH PURITY, SPECTROGRAPHIC GRADE

DIA IN.

$1 / 8$

$3 / 16$

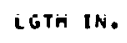

12
12

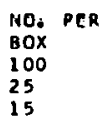

ROD, Flexaframe, aluminum, No. 14-666-10, fisher

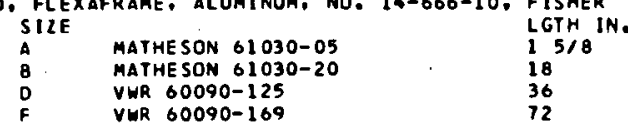

ROD. GLASS, 4 FI LGIH, JU LB PER PKG, O.4 MM TOTAL OD VARIATION

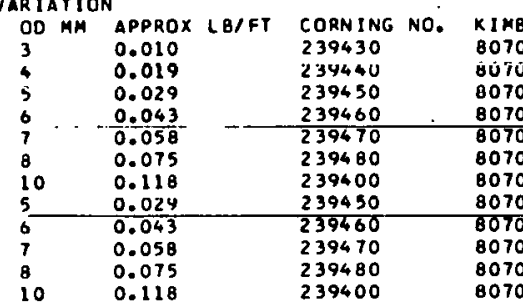

\begin{tabular}{|c|c|c|c|c|}
\hline EA & .00 & 3 & $15-183-2207$ & 36 \\
\hline EA & .00 & 23 & $15-183-2212$ & 36 \\
\hline EA & .00 & 3 & $15-183-2217$ & 36 \\
\hline EA & .60 & 23 & $15-183-2222$ & 36 \\
\hline EA & .00 & 3 & $15-183-2227$ & 36 \\
\hline $\begin{array}{l}\text { EA } \\
\text { EA } \\
\text { EA A }\end{array}$ & $\begin{array}{l}.08 \\
.09 \\
.16\end{array}$ & $\begin{array}{l}2 \\
2 \\
2\end{array}$ & $\begin{array}{l}15-183-2417 \\
15-183-2422 \\
15-183-2427\end{array}$ & $\begin{array}{l}23 \\
23 \\
23\end{array}$ \\
\hline LB & 5.16 & 3 & $15-183-4007$ & 36 \\
\hline CUFT & 172.32 & 1 & $15-183-4087$ & 36 \\
\hline CONT & 47.96 & 2 & $15-183-4097$ & 36 \\
\hline CUFT & 5.72 & 2 & $15-183-4167$ & 36 \\
\hline $\begin{array}{l}\text { EA } \\
\text { EA } \\
\text { EA } \\
\text { EA }\end{array}$ & $\begin{array}{l}.00 \\
.00 \\
.00 \\
.00\end{array}$ & $\begin{array}{r}3 \\
3 \\
23 \\
23\end{array}$ & $\begin{array}{l}15-183-6007 \\
15-183-6012 \\
15-183-6017 \\
15-183-6022\end{array}$ & $\begin{array}{l}36 \\
36 \\
36 \\
36\end{array}$ \\
\hline EA & 4.00 & 3 & $15-183-8007$ & 36 \\
\hline EA & 5.24 & 3 & $15-183-8017$ & 36 \\
\hline EA & 5.49 & 3 & $15-183-8507$ & 36 \\
\hline $\begin{array}{l}E A \\
E A \\
E A\end{array}$ & $\begin{array}{r}.90 \\
.97 \\
1.01\end{array}$ & $\begin{array}{l}3 \\
3 \\
3\end{array}$ & $\begin{array}{l}15-188-0100 \\
15-188-0120 \\
15-188-0140\end{array}$ & $\begin{array}{l}91 \\
91 \\
91\end{array}$ \\
\hline $\begin{array}{l}\text { EA } \\
\text { EA } \\
\text { EA } \\
\text { EA }\end{array}$ & $\begin{array}{l}.00 \\
.98 \\
1.00 \\
2.46\end{array}$ & $\begin{array}{l}23 \\
23 \\
23 \\
1\end{array}$ & $\begin{array}{l}15-189-1000 \\
15-189-1010 \\
15-189-1020 \\
15-189-1030\end{array}$ & $\begin{array}{l}36 \\
36 \\
36 \\
36\end{array}$ \\
\hline
\end{tabular}


ROD, KOVAR, CENTERLESS GROUND FINISH, IN 1 OR 2 FT STRAIGHT LGTHS. PLUS OR MINUS 0.001 IN., STUPAKOFF DIA IN.

MFR NO.

$$
0.060
$$

ROD, QUARTZ, FUSED, TRANSPARENT, 36 IN. LGTH OD
3
4
5
7
10

ROO, STIRRER, GLASS, GROUNO FULL LGTH, STO BUTTON TYPE. NO. 8256. STYLE A. ACE GLASS CO

ROD, STIRRER, MONEL METAL, DOUBLE V SHAPE, $1 / 4$ IN. OIA $X 6$ IN. LGTH. NO. 60012-20. MATHESCN OR LAPINE 38366

SAODLES, BERYL, KNIGHT-HARE, 1 1/2 IN., MaURICE A KNIGHT

SADOLES, PQRCELAIN, SIZE I IN. NOMINAL, RORTON CO INTALOX

SARAN WRAP, 12 IN. WIOTH, 50 FT ROLL, DOW CHEMICAL CO FSN B135-00-290-0338 GSA

SCale, metric and english, celluloto, heavy gauge, CALE, METRIC AND ENGLISH, CELLULOIO, HEAVY GAUGE, 6 IN. LGTH, GRADUATED IN $1 / 16$ IN. ON ONE EDGE AND REVERSE SIDE HAS TEMP CONVERSION SCALE FROM MINUS REVER SE SIDE HAS TEMP CONVERSION 100 DEG C AND MINUS 10 TO PLUS 212 DEG 25 TO PLUS 100 DEG C AND MINUS 10 TO PLUS 212 DEG F TOGETHER WIIH SCALES FOR DETER MINING SILES OF CORKS ANO RUBBER STOPPERS, NO.

SCALE, METRIC ANO ENGLISH, MAPLE, VARNISMEO, 36 IN. LGTH ONE SIDE GRAOUATEO IN MEIRIC SYSTEM SUBOIVIDEO TO MM BOTH EOGES. REVERSE SIDE GRADUATED IN INCHES SUBOIVIOED IN $1 / 8$ IN. BOTH EOGES, NO. 32940-10. MATHESON OR VWR 36525-009

SCALE. METRIC ANO ENGLISH, PLASTIC, TRANSPARENT, 12 IN. LGTH. GRAOUATED MM AND $1 / 16$ IN.. C-THRU RULER CO. PRE ISER 13-3530/01

SCISSORS, DISSECTING. CURVED, FINE POINTS, 25 MM LGTH BLADES. OVERALL LGTH 115 MM. NO. 19740-10, MATHESON OR VWR 25872-0OB

SCISSORS. DISSECTING, MAYO TYPE, STRAIGHT, NO. B1064. A S ALOE. SCIENT. PROD. D2650 OR APPROVED En!Ia!

SCISSORS, DISSECTING, SURGICAL OUALITY, ONE SHARP AND ONE BLUNT POINT, 50 MM LGTH BLADES, OYERALL LGTH 140 MA. MATHESON 19770-20 OR LAPINE $11070-02$

SCISSORS, NOQLE. MAYO TYPE, CURVED, STAIALESS STEEL H I/2 IN. A S ALOE, FSCO 13-804-12, OR APPROVED EQUAL

SCISSORS, SERRATED, STAINLESS STEEL, S-B $140 \mathrm{~mm}$ 5 1/2 IN.. NO. 38484, A S ALOE. NO. MX5-72, MLLTEX. OR APPROVEO EQUAL

SCISSORS, SERRATEO, STAINLESS STEEL, B-B 170 MM. $63 / 4$ IN.. NO. 38494, A S ALOE, NO. MX3-132, MILTEX, NO. MX 5-132 PRE ISER, OR AP PROVEO EQUAL

SCISSORS, STRABISMUS, STRAIGHT, STAINLESS STEEL, 115 MM, A S ALOE C-2735. PRETSER MX 18-1450, OR APPRO A S ALOE EOUAL

SCISSORS, STRAIGHT, STAINLESS STEEL, BLUNT AND BLUNT, $41 / 2$ IN.. A S ALOE B-1073A. AMER. MED, I5AM-200. FSCO $13-80 B$ OR APPROVED EOUAL

SCISSORS, STRAIGHT, STAINLESS STEEL, SHARP AND BLUNT, 1/2 IN.. A S ALOE B-1073C. AMER. MED. 15AM-100. F 1/2 IN.. A S ALOE B-1073C. AMER.

SCOOP. WEIGHING, COUNTERPOISEO, ALUMINUM WITH HANDLE. SILE $30 \mathrm{ML}$. FSCO 2-190 OR SARGENT S-3875B

SCOOPULA. STAINLESS STEEL, WITH ONE POINTEO END AND ONE BLUNT ENO. CURVED SHAPE, $61 / 2$ IN. LGTH. NO. 14-357. FISHER. NO. MS9150-10, MATHESON

SARGENT S-75290
Attachment B

UNIT

PRICE STK PT CATALOG NO BUYER

F r

IN

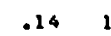

$15-189-6600$

36

IN IN

.03
.04
.06
.13
.23

$\begin{array}{ll}2 & 15-189-7600 \\ 23 & 15-189-7610 \\ 2 & 15-189-7620 \\ 3 & 15-189-7625 \\ 23 & 15-189-7630\end{array}$

97
97
97
97

$3 \quad 15-202-9880$

E A

$$
.00
$$

3

CUFT

CUF T

6.20

2

$15-189-8600$

36

36

ROLL

10.59

.35

23

15-195-1000 14

EA

.01

$15-196-2108$

36

$15-196-2128$

36

EA

.00

2

$15-196-2148$

36

EA

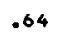

123

$15-196-2408$

36

EA

3.59

$15=196-2455$

EA

1.62

123

$15-196-2508$

36

E A

2

$15-196=2028$

F.

EA

EA

EA

EA

EA

EA

\section{$3.942 \quad 15-196-2648 \quad 36$}

$9.472 \quad 15-196-2668 \quad 36$

$9.15 \quad 2 \quad 15-196-2688 \quad 36$

$4.20 \quad 2 \quad 15-196-2708 \quad 36$

$4.20 \quad 2 \quad 15-196-2728 \quad 36$

$.003 \quad 15-196-4508 \quad 36$

$.00 \quad 1235 \quad 15-196-4608 \quad 36$ 
Attachment B

SCOOPULA HANDLE, WOOO, NO. L4-358, FOR ATTACHMENT TO NO. 14-357 SCOOPLA, FISHER, NO. 59152-10, MATHESON SEAL, BREAK, GLASS, 10 MM OIA, NO. 7734, CORNING PYREX

SEAL, GRAOED, PYREX GLASS NO. 7140 TO SOFTER COMPOSITION GLASS. 300 MM LGTH. NO. 6460. CORNING

$\begin{array}{lll}\text { OD MM } & \\ \text { iD (VWR } & 32843-280) \\ \text { 10 (VWR } & 32843-316)\end{array}$

10
13

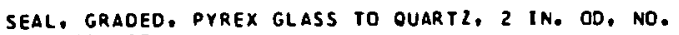
OGS5Ó. GE

SEAL, GRADED, KOVAR TO PYREX GLASS, STUPAXOFF KOVAR PYREX OVERALL MFR

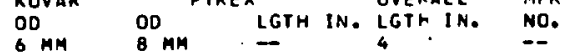

-- $1 / 4$ IN. $211237 / 8$ 9048-6, $C 00$

1/2 IN. 1/2 IN. $21 / 2$ 37/8 $\begin{aligned} & 9048-7, \text { COOE } \\ & 96-1009\end{aligned}$

3/8 IN. $10 \mathrm{MM} 22 \quad 3 \quad 9048$, COOE

$5 / 8$ IN. $16 \mathrm{MM} \quad 13 / 4 \quad 3 \quad 9048-10$

$9048-10$
CODE $96-1017$

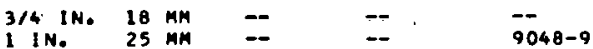

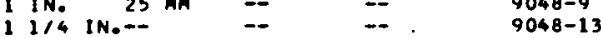

$11 / 2 \quad 11 / 2$ 9048-17,

IN. IN.

$131 / 32 \quad 9803$, CODE

IN. 2 IN. $11 \quad 14 \quad 96-1012$

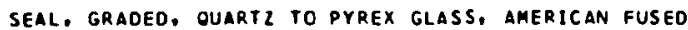
QUARTZ CO

ID IN

118

$3 / 8$

$\frac{1}{3} / \frac{2}{4}$

1

$1.1 / 4$

\begin{tabular}{|c|c|c|c|c|}
\hline EA & .00 & 3 & $15-196-4708$ & 36 \\
\hline EA & .91 & 23 & $15-199-2102$ & 36 \\
\hline $\begin{array}{l}\text { EA } \\
\text { EA } \\
\text { EA }\end{array}$ & $\begin{array}{r}2.31 \\
8.00 \\
10.45\end{array}$ & $\begin{array}{l}3 \\
3 \\
3\end{array}$ & $\begin{array}{l}15-199-2602 \\
15-199-2612 \\
15-199-2622\end{array}$ & $\begin{array}{l}36 \\
36 \\
36\end{array}$ \\
\hline EA & 30.00 & 3 & $15-199-2662$ & 97 \\
\hline EA & 2.88 & 3 & $15-199-4202$ & 36 \\
\hline EA & 2.82 & 123 & $15-199-4302$ & 36 \\
\hline EA & 3.77 & 123 & $15-199-4402$ & 36 \\
\hline EA & 3.40 & 123 & $15-199-4452$ & 36 \\
\hline EA & 4.90 & 3 & $15-199-4502$ & 36 \\
\hline $\begin{array}{l}\text { EA } \\
\text { EA } \\
\text { EA }\end{array}$ & $\begin{array}{r}4.68 \\
5.05 \\
15.05\end{array}$ & $2_{3}^{23}$ & $\begin{array}{l}15-199-4567 \\
15-199-4602 \\
15-199-4652\end{array}$ & $\begin{array}{l}36 \\
36 \\
36\end{array}$ \\
\hline EA & 8.75. & 2 & $15-199-4657$ & 36 \\
\hline$E A$ & 12.45 & 23 & $15-199-4662$ & 36 \\
\hline
\end{tabular}

SEAL, GRADED, YYCOR TO PYREX GLASS, NO, 6466, CORNING OD MM

9

15

51

$\begin{array}{lllll}\text { EA } & 4.18 & 3 & 15-199-6202 & 97 \\ \text { A } & 4.41 & 3 & 15-199-6207 & 97 \\ A & 5.43 & 3 & 15-199-6212 & 97 \\ \text { EA } & 10.35 & 3 & 15-199-6217 & 97 \\ \text { EA } & 13.35 & 3 & 15-199-6222 & 97 \\ \text { EA } & 17.16 & 3 & 15-199-6227 & 97 \\ & 16.80 & 3 & 15-199-6232 & 97 \\ & & 15-199-6237 & 97\end{array}$

SEAL, HOUSEKEEPER, OHFC, COPPER TO PYREX GLASS, COPPER g IN. LCTH, PYAE GLASS G IH. LGTH, VACTRONIC RESEARCH

DIA IN.

114

Sealant, leak, high VacuUm, capable of attaining ULTIMATE PRESSURES OF $2 \times 10$ MI NUS 12 TORR, 1602 AEROSOL CAN, SPACE ENVIRONMENT LABORATORIES

SHAFT. STIRRER, PYREX GLASS, STYLE A, FOR ACE GLASS STIRRER NO, 8245, ACE 8134-25, OR SGA NO. JS-2040, $250-5000 \mathrm{ML}$

SHAPER, GLASS BLOMERS, CARBON, SILE $20 \times 25 \mathrm{MM}$, NO. $27494-20$, MATHESON OR FSCO $11-346-30$

SHAPER, GLASS bloweas, CaRBON, heXaconal TAPERgo ROD, 150 MM LGTH, SMALL OIA I MM, LARGE DIA 15 KH, ROD, 250 MH LGTH, SMALL OIA 1 NH. CARGE DIA

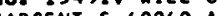

$\begin{array}{rrrrr}\text { EA } & 2.73 & 3 & 15-199-6305 & 36 \\ \text { EA } & 4.47 & 3 & 15-199-6310 & 36 \\ \text { EA } & 11.60 & 3 & 15-199-6320 & 36 \\ \text { EA } & 20.02 & 3 & 15-199-6325 & 36\end{array}$

SHAPER, GLASS BLOMERS, FLARING, LONG FORM, BRASS DLOE YITH HOOD MANDLE BLADE WIDTH AT HAMOLE 37 OL AOE, WITH HOOD HANDLE, BLADE WIDTH AT HANOL MM. BLADE LGTH 75 MM
NO. $12-9225$. PREISER

$\begin{array}{lllll}\text { EA } & 5.05 & 3 & 15-199-6602 & 36 \\ \text { EA } & 6.49 & 3 & 15-199-6617 & 36 \\ & & & & \\ \text { CAN } & 53.49 & 2 & 15-199-7120 & 36 \\ & & & & \\ \text { EA } & 1.04 & 3 & 15-202-0500 & 36 \\ & .00 & 3 & 15-202-1110 & 36 \\ \text { EA } & 1.80 & 3 & 15-202-1300 & 36 \\ & & & & \\ \text { EA } & 2.60 & 3 & 15-202-1500 & 36\end{array}$


Attachment B

STOPCOCK, HIGH PRESSURE, PYREX GLASS, STRAIGHT GORE, ACE 8176 OR LABGLASS LG-10180-5

STOPPER BORE MM

3

STOPCOCK, HIGH VACUUM, PYREX GLASS, MERCURY SEAL AND

HOLLOW PLUG. FOR CONNECT ING EITHER RIGHT OR LEFT

OUTLET MBOTTOM OUTLET, ACE B141. LABGLASS LG-1008

STOPPER

BORE MM

4

$\begin{array}{lrlll}\text { EA } & 9.92 & 3 & 15-205-2010 & 36 \\ \text { EA } & 13.61 & 3 & 15-205-2020 & 36 \\ \text { EA } & 16.91 & 3 & 15-205-2030 & 36\end{array}$

STOPCOCK. HIGM VACUUM, PYREX gLASS, MERCURY SEAL,

RIGHT ANGLE, SGA J-450S OR LABGLASS LG-1007

STI ANGL
STPER

BORE MM

4

6
8
15

\begin{tabular}{lrlll}
$E A$ & 6.35 & 3 & $15-205-2400$ & 36 \\
$E A$ & 8.83 & 3 & $15-205-2405$ & 36 \\
$E A$ & 10.78 & 3 & $15-205-2410$ & 36 \\
\hline$E A$ & 10.78 & 3 & $15-205-2415$ & 36 \\
$E A$ & 12.98 & 3 & $15-205-2420$ & 36 \\
$E A$ & 30.64 & 3 & $15-205-2430$ & 36
\end{tabular}

STOPCOCK, HIGH VACUUM, PYREX GLASS, PRECISION GRAOE

WITH HOLLOH BLOWN STOPPER ANO STRAIGHT BORE. ND.

5000 , ECK AND KREBS

STOPPER

BORE MM

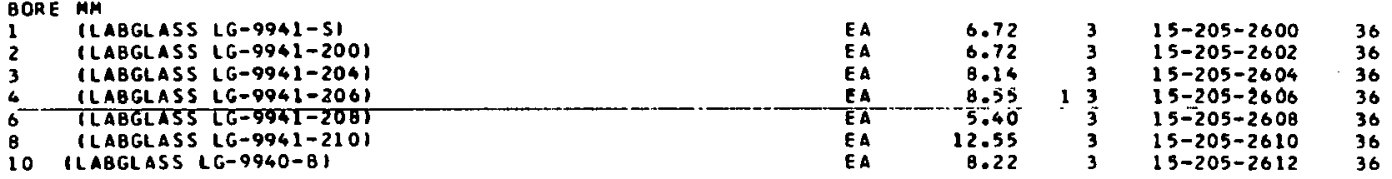

STOPCOCK, HIGH VACUUM, PYREX GLASS, WITH HOLLOW PLUG FOR HORIZONTAL CONN, STOPPER BORE 4 MM, NO. 8201-06 ACE GLASS

STOPCOCK. HIGH VACUUM, PYREX GLASS, PRECISION GRADE, WITHOUT MERCURY SEAL, FOR HORIZONTAL FLOW, NO.

5044. ECK AND KREBS

STOPPER

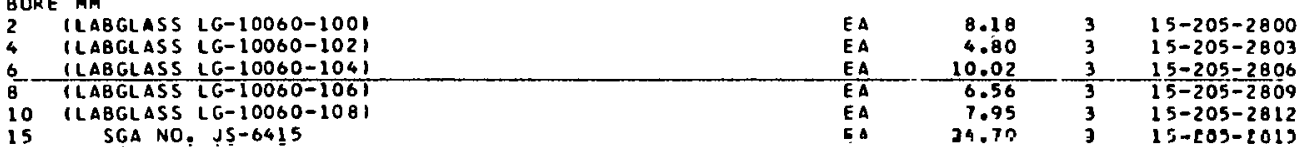

STOPCOCK, HIGH VACUUM, HORI ZONTAL FLOW, MOLLOH PLUG, 4 MM BORE. TAPER 17/40. SIOE ARMS $10 \mathrm{MF}$ OD. NO. LG-10OBO. LARGI AS TR FXAC.T EQUAL

STOPCOCK, MERCURY SEAL, PYREX GLASS, TWO-MAY, CAP-

ILLARY, SOLIO STOPPER PLUG, NO. 1500 , CORNING

STOPPER BORE MM

2

STOPCOCK, MICRO, KIMAX GLASS, HITH TEFLON PLUG, I TO 5 RATIO TAPER, PLUG BORE I MA, NO. 41011 F. KIMBLE

STOPCOCK, KIMAX GLASS, T-8ORE, WITH TEFLON PLUG, 1 TO 5 RATIO TAPER. PLUG BORE ? MM, NO. $41044 F$, KIMBLE

STOPCOCK, CAPILLARY, KIMAX GLASS, T-BORE, WITH TEFLON PLUG, i TO 5 katio TaPé, PLUG bCRE 1 i/2 MM. NO. 41049F. KIMBLE

STOPCOCK, OBLIOUE BORE, PYREX GLASS, SOL TO STOPPER PLUG. NO. 7340 . CORNINC

STOPPER BORE MM

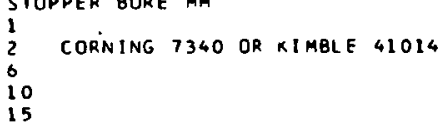

STOPCOCK, PRECISION GRADE, PYREX GLASS, WITHOUT MERCURY SEAL. WITH HOLLOW PLUG FOR RT ANGLE FLOW. NO. SOS4. ECK AND KREBS

STOPPER BORE MM

4 OR LABCLASS LG-10060-102

36

$\begin{array}{lcccc}\text { EA } & 9.82 & 2 & 15-205-2850 & 36 \\ & & & & \\ \text { EA } & 12.78 & 3 & 15-205-4500 & 36 \\ \text { EA } & 15-205-4505 & 36 \\ \text { EA } & 7.33 & 23 & 15-205-4600 & 36 \\ & 8.39 & 3 & 15-205-4620 & 36 \\ \text { EA } & 11.94 & 23 & 15-205-4640 & 36\end{array}$

$\begin{array}{lrlll}\text { EA } & 3.37 & 3 & 15-205-4820 & 36 \\ \text { EA } & 4.57 & 3 & 15-205-4822 & 36 \\ \text { EA } & 6.54 & 3 & 15-205-4828 & 36 \\ \text { EA } & 3.92 & 3 & 15-205-4832 & 36 \\ \text { EA } & 56.50 & 3 & 15-205-4835 & 36\end{array}$

$\begin{array}{lllll}\text { EA } & 4.80 & 3 & 15-205-5300 & 36 \\ \text { EA } & 9.00 & 3 & 15-205-5310 & 36\end{array}$ 
Attachment B

STOPCOCK, PRECISION GRADE, PYREX GLASS, WITHOUT MERCURY SEAL. WITH HOLLON PLUG WITH OBLIOUE BORE, NO. 5100, ECK AND KREBS

BORE MM

2 OR LABGLASS LG-10090-100

2 OR LABGLASS LG-10090

- OR LABGLASS LG-10090

STOPCOCK, PRECISION GRADE, PYREX GLASS, WITHOUT MERCURY SEAL, 3-WAY OBLIOUE BORE, WITH HOLLOW PLUG. ECK + KREBS 5124 OR LABGLASS LG-10110 STOPPER BORE MM

TOPCOCK, PRECISION GRAOE, PYREX GLASS, WITH 120 DEG ANGLE BORE SEALED INTC STOPPER, NO. 5032 , ECX - kREBS OR lagGlass LG-10040-B BORE MM

$$
2
$$

IOR SGA JS-6085I

STOPCOCX. PRECISION GRADE, PYREX GLASS, WITH T BORE SEALED INTO STOPPER, NO. LG-100OO-B LABGLASS BORE MM

$$
\begin{aligned}
& 2 \\
& 3 \\
& 4
\end{aligned}
$$

STOPCOCK, STRAIGHT BORE, GLASS, MITH TEFLON PLUG I TO 5 RATIO TAPER, CORNING 7282 , KIMQLE $41004-F$ OR 5 RATIO TAPER,
APPROVED EOUAL APPROVEO EOUAL

1

$11 / 2$$$
4
$$

STOPCOCK, STRAIGHT BORE, GLASS, TEFLON PLUG WITH METERING VALVE, I TO 5 RATIO TAPER, NO. $41002-F$ XIMBLE PLUG BORE MM 2

STOPCOCK, STRAIGHT BORE, CAPILLARY, BOROSILICATE GLASS, WITH TEFLON PLUG I TO 5 RATIO TAPER, CORNING 7302; KIMBLE 41009-F OR APPROVEO EOUAL BORE MM

$$
11 / 2
$$

STOPCOCK, STRAIGHT BORE, CAPILLARY, KIMAX GLASS, TEFLON PLUG WITH METERING VALVE, I TO 5 RATIO TAPER. PLUG BORE I $1 / 2$ MM. NO. $41007 \mathrm{~F}, \mathrm{KIMBLE}$

STOPCOCK, STRAIGHT BORE, CAPILLARY, BOROSILICATE GLASS. MITH SOL ID S/T STOPPER PLUG, CORNING 7300 OR XIMBLE 41009 BORE MM<smiles>[TeH]</smiles>
$11 / 2$

STOPCOCK, STRAIGHT BORE, PYREX GLASS, MICRO, SOLID STOPPER PLUG. INTERCHANGEABLE, APPRCX BDRE OF STOPPER PLUG, INTERCHANGEABLE, APPRCX BORE

STOPCOCK, STRAIGHT BORE, PYREX GLASS, WITH SOLIO SIT STOPPER PLUG, CORNING 7280 OR XIMBLE 41004 STOPPER

$$
1
$$$$
2
$$

$$
\begin{aligned}
& 3 \\
& 4 \\
& 6
\end{aligned}
$$

STOPCOEK, STRALGHT BORE, RYREX GLASS, WITM SOL TD SIT STOPPER PLUG, OO OF SIOE ARMS 12. MM, STOPPER BORE 6 MM, STOPPER NO. 6. NO. K-601000-0006. KONTES GLASS CO. NO SUB

\begin{tabular}{|c|c|c|c|c|}
\hline UNIT & PRICE & STK PT & CATALOG NO & BUYER \\
\hline & & & & \\
\hline $\begin{array}{l}E A \\
\text { EA } \\
\text { EA }\end{array}$ & $\begin{array}{l}7.69 \\
7.69 \\
9.31\end{array}$ & $2^{3}$ & $\begin{array}{l}15-205-5395 \\
15-205-5400 \\
15-205-5405\end{array}$ & $\begin{array}{l}36 \\
36 \\
36\end{array}$ \\
\hline EA & 15.78 & 3 & $15-205-5450$ & 36 \\
\hline $\begin{array}{l}\text { EA } \\
\text { EA } \\
\text { EA }\end{array}$ & $\begin{array}{l}6.11 \\
6.20 \\
6.78\end{array}$ & $\begin{array}{l}3 \\
3 \\
3\end{array}$ & $\begin{array}{l}15-205-5600 \\
15-205-5605 \\
15-205-5610\end{array}$ & $\begin{array}{l}36 \\
36 \\
36\end{array}$ \\
\hline $\begin{array}{l}E A \\
E A \\
E A\end{array}$ & $\begin{array}{l}6.20 \\
8.34 \\
8.82\end{array}$ & $\begin{array}{l}3 \\
3 \\
3\end{array}$ & $\begin{array}{l}15-205-5800 \\
15-205-5805 \\
15-205-5810\end{array}$ & $\begin{array}{l}36 \\
36 \\
36\end{array}$ \\
\hline $\begin{array}{l}\text { EA } \\
\text { EA } \\
\text { EA } \\
\text { EA }\end{array}$ & $\begin{array}{r}6.79 \\
5.80 \\
5.98 \\
9.62 \\
11.39\end{array}$ & $\begin{array}{r}3 \\
3 \\
23 \\
23 \\
23\end{array}$ & $\begin{array}{l}15-205-6050 \\
15-205-6055 \\
15-205-6060 \\
15-205-6065 \\
15-205-6070\end{array}$ & $\begin{array}{l}36 \\
36 \\
36 \\
36 \\
36\end{array}$ \\
\hline $\begin{array}{l}\text { EA } \\
\text { EA }\end{array}$ & $\begin{array}{l}14.42 \\
15.91\end{array}$ & $\begin{array}{r}3 \\
23\end{array}$ & $\begin{array}{l}15-205-6080 \\
15-205-6085\end{array}$ & $\begin{array}{l}36 \\
36\end{array}$ \\
\hline $\begin{array}{l}\text { EA } \\
\text { EA } \\
\text { EA }\end{array}$ & $\begin{array}{l}6.34 \\
6.43 \\
7.12\end{array}$ & $\begin{array}{r}3 \\
23 \\
23\end{array}$ & $\begin{array}{l}15-205-6220 \\
15-205-6225 \\
15-205-6230\end{array}$ & $\begin{array}{l}36 \\
36 \\
36\end{array}$ \\
\hline EA & 14.86 & 3 & $15-205-6233$ & 36 \\
\hline $\begin{array}{l}\text { EA } \\
\text { EA } \\
\text { EA }\end{array}$ & $\begin{array}{l}3.94 \\
4.18 \\
4.37\end{array}$ & $\begin{array}{r}3 \\
23\end{array}$ & $\begin{array}{l}15-205-6250 \\
15-205-6255 \\
15-205-6260\end{array}$ & $\begin{array}{l}36 \\
36 \\
36\end{array}$ \\
\hline EA & 4.90 & 3 & $15-205-6300$ & 36 \\
\hline $\begin{array}{l}E A \\
\text { EA } \\
\text { FA } \\
\text { EA } \\
\text { EA }\end{array}$ & $\begin{array}{l}3.48 \\
4.18 \\
4.91 \\
5.45 \\
6.30\end{array}$ & $\begin{array}{l}3 \\
235 \\
23 \\
3 \\
3\end{array}$ & $\begin{array}{l}15-205-6400 \\
15-205-6402 \\
15-205-6404 \\
15-205-6406 \\
15-205-6408\end{array}$ & $\begin{array}{l}36 \\
36 \\
36 \\
36 \\
36\end{array}$ \\
\hline & & & & · \\
\hline EA & 7.67 & 5 & $15-205-6512$ & 36 \\
\hline $\begin{array}{l}\text { EA } \\
\text { EA } \\
\text { EA }\end{array}$ & $\begin{array}{r}9.80 \\
11.82 \\
14.78\end{array}$ & $\begin{array}{l}3 \\
3 \\
3\end{array}$ & $\begin{array}{l}15-205-7900 \\
15-205-7905 \\
15-205-7910\end{array}$ & $\begin{array}{l}36 \\
36 \\
36\end{array}$ \\
\hline
\end{tabular}

STOPCOCK. THREE-HAY, KIMAX GLASS, WITH TEFLON PLUG I TO 5 RATIO TAPEA, KIMBLE 41024F, CORNING 7382, OR APPROVEO EOUAL

BOR E MH<smiles>[Te][Te]</smiles> 
Attachment B

STOPCOCK, THREE-WAY, KIMAX GLASS, 120 DEG BORE, WITH TEFLON PLUG, 1 TO 5 RATIO TAPER, BORE FLUG 2 MM, NO. $41055 F$, KIMBLE

STOPCOCK, THREE-WAY, CAPILLARY, BOROS ILICAJE GLASS, WITH TEFLON PLUG 1 TO 5 RATIO TAPER. CCRNING 7402. KIMBLE 41029-F OR APPROVED EOUAL

BORE MM

$11 / 2$

112

STOPCOCK. THREE-WAY, PYREX GLASS, 120 OEG 8ORE,

SOL ID STOPPER PLUG, CORNING 7450 OR KIMBLE 41055

STOPPER BORE MH SIOE ARMS DO MM

$\begin{array}{lc}\text { STOPPER BORE MH } & \text { SIOE ARMS } \\ 2 & 8 \\ 4 & 10\end{array}$

STOPCOCK, THREE-WAY, PYREX GLASS, CAPILLARY, SOLIO STOPPER PLUG. 7 MM OD SIDE ARMS, CORNING 7400 OR STOPPER PLUC.

STOPPER BORE MM

$$
11 / 2
$$

STOPCOCK, THREE-WAY, PYREX GLASS, MERCURY SEAL, SOL ID STOPPER PLUG, 2 ARMS ON ONE SIDE ANO 1 ARM OPPOSITE. NO. 7540, CORNING

$\begin{array}{ll}\text { STOPPER BORE } & \text { OO SIOE } \\ \text { MH } & \text { ARMS MM } \\ 2 & B\end{array}$

STOPCOCK. IMREE-WAY, PYREX GLASS, SOL IO STOPPER PLUG, WITH 2 ARMS ON 1 SIDE ANO 1 ARM OPPOSITE. NO. 380. CORNING OR KIMBLE 41024 STOPPER BORE HM

1

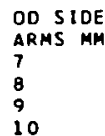

10

STOPCOCK, THREE-WAY, PYREX GLASS, T SHAPE, CAPILLARY. 7 MM OO SIDE ARMS, SOLID STOPPER PLUG,. NO. 7440, CORNING OR KIMBLE 41049

STOPPER BORE MM$$
2
$$

STOPCOCK, THREE-WAY, PYREX GLASS, T SHAPE, SOLID STOPPER PLUG, CORNING 7420 OR KIMBLE 41044

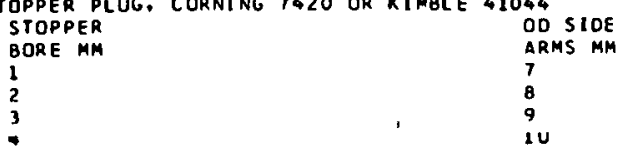

STOPPER, CORK, XXX OUALITY, FLAT, EXTRA SHORT, NO, 5-23105, SARGENT, OR MANTON

-23105,

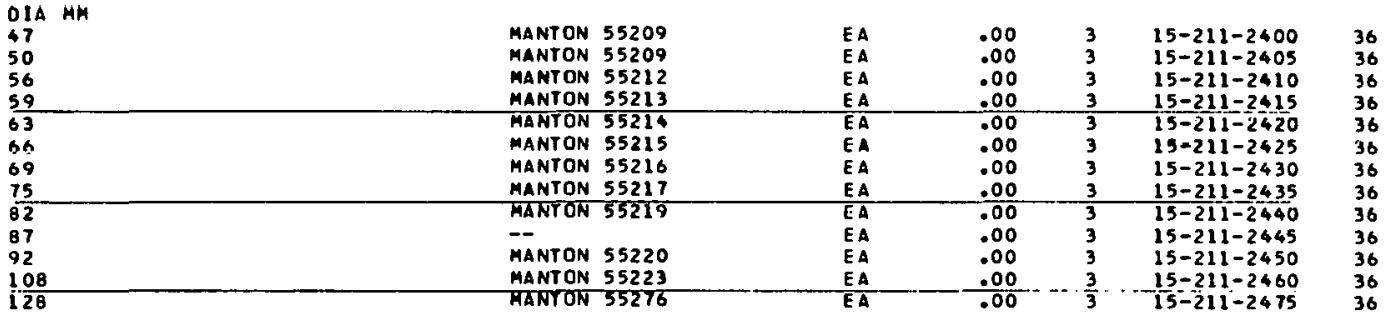

STOPPER, CORK, XXXX OUALITY, LABORATORY, REGULAR

LGTH

$\begin{array}{llll}\text { LTH } & & \text { TIZE LGTH } & \text { TOP OIA } \\ \text { NO. } & \text { MM } & \text { MHOM DIA } & \end{array}$

$0000 \quad--\quad--\quad$ SARGENT XS-23025-X
SCIENTIFIC PROOUCTS CB220-4T

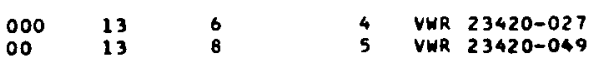

$013 \quad 11 \quad 7$ MATHESON 16810-05

13 11 SCI. PROD. C8205-1T

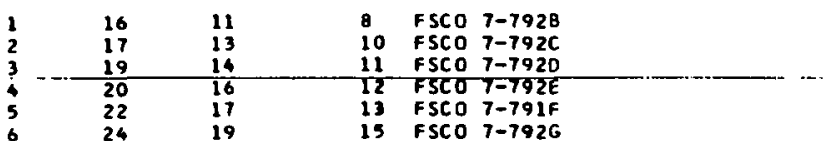

$\begin{array}{lcccc}\text { EA } & .00 & 23 & 15-211-2802 & 36 \\ \text { EA } & .00 & 123 & 15-211-2804 & 36 \\ \text { EA } & .00 & 123 & 15-211-2806 & 36 \\ & & & & \\ \text { EA } & .01 & 123 & 15-211-2808 & 36 \\ \text { EA } & .00 & 123 & 15-211-2810 & 36 \\ \text { EA } & .00 & 23 & 15-211-2812 & 36 \\ \text { EA } & .00 & 235 & 15-211-2814 & 36 \\ \text { EA } & .00 & 123 & 15-211-2816 & 36 \\ \text { EA } & .00 & 1235 & 15-211-2818 & 36 \\ \text { EA } & .00 & 13 & 15-211-2820 & 36\end{array}$

EA $\quad 5.49 \quad 3 \quad 15-205-8454 \quad 36$

$\begin{array}{lrrrr}\text { EA } & 3.80 & 3 & 15-205-8700 & 36 \\ \text { EA } & 4.67 & 23 & 15-205-8702 & 36 \\ \text { EA } & 5.47 & 3 & 15-295-8704 & 36 \\ \text { EA } & 7.57 & 3 & 15-205-8706 & 36\end{array}$


TUBE, ALUNDUM. TYPE RA9B, SPIRALLY GROUND, NORTON BORE IN. PITCH IN. LGTH IN. MFR NO. $\begin{array}{llll}2 & 118 & 10 & 6916 \\ 3112 & 5 / 16 & 5 & 6366\end{array}$

TUBE, CENTRIFUGE, CELlULOSE NITRATE, SILE $1 / 2$ IN. $X$ 2 IN.. NO. 30SOSO, BECKMAN

TUBE, CENTRIFUGE, CONICAL BOTTOM, GRAOUATED, SCREW CAP. $15 \mathrm{ML}$ SILE. GRADUATEO IN $0.1 \mathrm{ML}$ DIVISIONS RUBBER LINER CEMENTEO IN CAPS, KIMAX NC. 45166, KIMBLE

TUBE, CENTRIFUGE, GLASS, TAPER BOTTOM, GRAOUATEO, HEAVY DUTY, $40 \mathrm{ML}$ CAP., NO. CY-2055-H, CLAY-ADAMS CO

TUBE, CENTRIFUGE, POLYPROPYLENE, MOLDED, RIMLESS ROUND BOTTOM, 50 ML CAP., 29 MM CD, 104 MM LGTH. KIMBLE 58350

TUBE. CENTRIFUGE, POLYETHYLENE

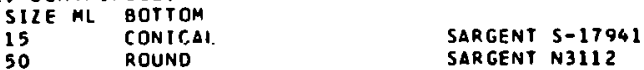

IUBE, CENTRIFUGE, POL YPROPYLENE, $100 \mathrm{ML}$ CAP.. NO. 5-562-10. FSCO. MATHESON 10200-32 OR VHR 60B17-325

TUBE, CENTRIFUGe, PYrex glass, CONICAL, eEaded RIM. GRADUATED. CORNING BOBO OR KIMBLE 45165 CAP. ML

15
50

TUBE, CENTRIFUGe, PYKEX GLASS, CONICAL, BEADED RIM, MEAYY DUTY, GRADUATED. CORNING BI4O OR KIMBLE 4519 CAP. ML 12

TUBE. CENTRIFUGE, PYREX GLASS, CONICAL, EEAOED RIM, HEAVY DUTY. CORNING 8120 OR KIMBLE 45194$$
\text { CAP. ML }
$$

12
40

TUBE, CENTRIFUGE, PYREX GLASS, CONICAL, GEADEO RIM. PLAIN, NO, 8060, CORNING

CAP. ML

CAP. ML KIMBLE 45150

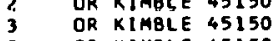

I5 OR KIMBLE 45150

TUBE, CENTRIFUGE, PYREX GLASS, GRADUATED IN $1 / 10$ ML OIVISIONS, WITHOUT HOOXS, 14/15 S/T GROUND JOINTS, FLAT-HEAOED STOPPER, $14 / 15$ S/T WITHOUT HOOKS, 10 ML CAP.. 16.5 TO $17.0 \mathrm{MM} \mathrm{OD,} 0.650$ TO $0.669 \mathrm{OD}$. NO. JT-7T10. SGA. LABGLASS SPECIAL OR APPROVEO EOUAL

TUBE, CENTRIFUGE, PYREX GLASS, HEAYY DUTY, SHORT CONICAL BOTTOM, UNGRADUATED, $40 \mathrm{ML}$. NO, 8320 , CORN ING

TUBe, CENTRIFUCE, PYREX GLASS, OIL, PEAR SHAPE, STEM GRADUATEO O TO 1.5 ML IN O.I OIV, BODY GRADUGRADUATEO O TO $1.5 \mathrm{ML}$ IN 0.1 OIV, BODY GRADUATED FROM 1.5 ML TO $100 \mathrm{ML}$ SPEC ASIM D96, $100 \mathrm{ML}$ CAP.. NO. 8200 . $100 \mathrm{ML}$, SPEC ASTM D96.

TUBE. CENTRIfUGe, PYREX GLASS, gOETZ, PEAR SHAPE, GRADUATEO, LOWER STEM GRADUATEO UPWARO TO $1 \mathrm{ML}$ IN 0.05 DIV. BODY GRADUATED FROM I ML TO 5 ML IN I ML OIV, FROM $5 \mathrm{ML}$ TO $50 \mathrm{~mL}$ IN $5 \mathrm{ML}$ OIV. FROM $50 \mathrm{ML}$ TO $100 \mathrm{ML}$ IN $10 \mathrm{ML}$ DIV, $100 \mathrm{ML}$ CAP., NO. 8220 CORNING OR KIMBLE 45250

TUBE, CENTRIFUGe, PYREX glass, ROUNO BOTTOH, hEAVY DUTY WITH LIP, SO ML CAP.. PER ORNL SKETCH NO A-2. H S MAKI IN

TUBE, CENTRIFUGE, PYREX GLASS, ROUND BOTTOM, POUROUT. PLAIN, CORNING 8240 OR KIMBLE 45190

CAP. ML

50

TUBE, CENTRIFUGE, POL YPROPYLENE, 11 MM O1A $\times 71 \mathrm{MM}$ LGTH. MATHESON 20206-06
At tachment B

\begin{tabular}{|c|c|c|c|c|}
\hline UNIT & PRICE & STK PI & CATALOG NO & BUYER \\
\hline $\begin{array}{l}E A \\
\text { EA }\end{array}$ & $\begin{array}{r}18.80 \\
.00\end{array}$ & $\begin{array}{l}3 \\
3\end{array}$ & $\begin{array}{l}15-242-0733 \\
15-242-0743\end{array}$ & $\begin{array}{l}36 \\
36\end{array}$ \\
\hline EA & .20 & 2 & $15-242-0900$ & 36 \\
\hline EA & 2.22 & 3 & $15-242-1093$ & 36 \\
\hline EA & .00 & 3 & $15-242-1163$ & 36 \\
\hline EA & .15 & 3 & $15-242-1225$ & 36 \\
\hline $\begin{array}{l}E A \\
E A\end{array}$ & $\begin{array}{r}.28 \\
.25\end{array}$ & $\begin{array}{l}2 \\
2\end{array}$ & $\begin{array}{l}15-242-1703 \\
15-242-1753\end{array}$ & $\begin{array}{l}36 \\
36\end{array}$ \\
\hline EA & .52 & 3 & $15-242-1950$ & 36 \\
\hline $\begin{array}{l}E A \\
E A\end{array}$ & $\begin{array}{l}1.51 \\
1.90\end{array}$ & $\begin{array}{r}123 \\
23\end{array}$ & $\begin{array}{l}15-242-2153 \\
15-242-2163\end{array}$ & $\begin{array}{l}36 \\
36\end{array}$ \\
\hline $\begin{array}{l}E A \\
E A\end{array}$ & $\begin{array}{l}1.58 \\
2.35\end{array}$ & $\begin{array}{l}2 \\
2\end{array}$ & $\begin{array}{l}15-242-2183 \\
15-242-2193\end{array}$ & $\begin{array}{l}36 \\
36\end{array}$ \\
\hline $\begin{array}{l}E A \\
\text { EA }\end{array}$ & $\begin{array}{r}.41 \\
.74\end{array}$ & $\begin{array}{l}3 \\
3\end{array}$ & $\begin{array}{l}15-242-2203 \\
15-242-2213\end{array}$ & $\begin{array}{l}36 \\
36\end{array}$ \\
\hline $\begin{array}{l}\text { EA } \\
\text { EA } \\
\text { EA } \\
\text { EA }\end{array}$ & $\begin{array}{l}.33 \\
.34 \\
.31 \\
.37\end{array}$ & $\begin{array}{r}2 \\
23 \\
3 \\
23\end{array}$ & $\begin{array}{l}15-242-2263 \\
15-242-2273 \\
15-242-2283 \\
15-242-2293\end{array}$ & $\begin{array}{l}36 \\
36 \\
36 \\
36\end{array}$ \\
\hline EA & 3.44 & 2 & $15-242-2343$ & 36 \\
\hline EA & .02 & 3 & $15-242-2353$ & 36 \\
\hline EA & 4.44 & 3 & $15-242-2373$ & 36 \\
\hline EA & 4.77 & 23 & $15-242-2393$ & 36 \\
\hline CA & .60 & 3 & $15-342-2403$ & 35 \\
\hline $\begin{array}{l}E A \\
\text { EA }\end{array}$ & $\begin{array}{l}.52 \\
.74\end{array}$ & $\begin{array}{ll}13 & 3 \\
3\end{array}$ & $\begin{array}{l}15-242-2423 \\
15-242-2428\end{array}$ & $\begin{array}{l}36 \\
36\end{array}$ \\
\hline$E$ & 04 & 3 & $15-242-2453$ & 30 \\
\hline
\end{tabular}


TUBE. CENTRIFUGE, POL YPROPYLENE, PLAIN

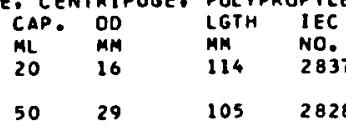

MATHESON 10206-20

TUBE, CENTRIFUGE, POLYPROPYLENE, ROUNO BOTTOM. PLAIN, 10 ML CAP.. 127 MH $\times 108$ MM. PRE ISER 16-8363,

MATHESON $10206-12$ OR VHR 21011-275

TUBE, COMBUSTION. MCOANEL, HIGH TEMP, VITREOUS,

SINGLE REDUCED END. BURRELL CORP A24-510

IO OD OVERALL
IN. OT.

\begin{tabular}{|c|c|c|c|}
\hline $11 / 8$ & $13 / 8$ & 30 & $\begin{array}{r}\text { MATHESON } 15360-40 \\
\text { OR F SCO } 7-586 F\end{array}$ \\
\hline $\begin{array}{ll}1 & 1 / 4 \\
1 & 3 / 4\end{array}$ & $1_{2}^{1} 1 / 2$ & $\begin{array}{l}33 \\
36\end{array}$ & $\begin{array}{r}\text { MATHESON } 15360-60 \\
\text { WLL CTT13436 }\end{array}$ \\
\hline
\end{tabular}

TUBE. COMBUSTION. PYREX, CLOSED END, 15 MM 10, 20 MM OD, 600 MM LGTH, NO. 8620. CORNING

TUBE, COMBUSTION, VYCOR BRAND GLASS, SIZE C, 1000 MM LGTH $\times 19 \mathrm{MM}$ ID $\times 25$ MM OD. NO. 18600, CORNING

TUBE, CONNECTION, GLASS, NO. GIO428A. EMIL GREINER NO. 78-426-01. IBO MMI. MANOSTAT Nor $T=8$ 17449-002

TUBE, CONNECTING, BOROSILICATE GLASS, 75 DEG, IWO WAY UITH INNER SIT JOINTS OF THE SAME SIZE, SIT 19138 . CORNING 8920 OR KIMBLE 44920

TUBE, CONNECTING, BOROSILICATE GLASS, 75 DEG, TWOWAY, WITH INNER S/T JOINTS OF THE SAME SILE, SIT WAY, WITH INNER S/T JOINTS OF THE SAM
$24 / 40$. CORNING 8920 OR KIMBLE 44920

TUBE, CONNECTING, BOROSILICATE GLASS, 105 OEg. THOWAY. S/T NO. $24 / 40$, WITH INNER AND OUTER JOINT, NO. 8940 . CORNING OR KIMBLE 44925

TUBE, CONNECTING. T-SHAPE, GLASS, WITH CCNSTRICTED ENDS, FOR RUBBER TUBING. CORNING 9187 -KIMBLE 45020 TO FIT TUBING APPROX OO LGTH LGTH HAVING INSIDE OF TUBE CROSSPIECE UPRIGHT $3 / 16$ O 6 TOBE

$1 / 4$

$3 / 8$

88
88
120
140

44
60

TUBE, CONNECTING, Y-SHAPE, GLASS, MITH CCNSTRICTED ENDS, FOR RUABER TUBING. CORNING 9189 -KIMBLE 45030 TO FIT TURING APPROX DR IGTH HAVING INSIDE OF TUBE EACH ARM DIA IN.

114 MM

TUBE, CORNELL STYLE, METAL, 3/4 IN, DIA $\times 41 / 2$ IN. LGTH, NO. 302. INTERNATIONAL EOUIPMENT CO OR NO. $9087-10$. MATHESON

TUBE, CORNELL STYLE, METAL, $13 / 8$ IN. DIA $X 6$ IN. LGTH. NO. 340, INTERNATIONAL EOUIPMENT CO OR ND. 9100-10. MATMESON

TUBE. DRINKING. PYREX GLASS, 8.50 TO $8.75 \mathrm{MM}$ DIA WITH 1 MM WALL. LONG END $21 / 4$ IN. LGTH. WITH 2 WITH I MM WALL. LONG 3 MM FIRE POLISHED OPENING, SHORT END $11 / 4$ IN. LGTH FIRE POLISHED ANO WITHOUT CONSTRICTION, IN. LGTH FIRE POL ISHED

TUBE, DRYING, CALCIUM CHLORIDE, SCMWARTZ, GLASS WITH SIT STOPPER NO. 14/20 AND CONSTRICTED ENOS FOR RUBBER TUBING. NO. 46050 , KI MBLE RUBBER TU 100 150

TUBE, ORYING, CALCIUM CHLORIDE, STRAIGHT FORM. GLASS, SINGLE BULA, WITH CCNSTRICTEO END FOR RUBBER TUBING. CORNING 7775 OR KIMBLE 46010

LGTH MM TO

BOTTOM OF BULB

150

TUBE, FILTER, BOROSILICATE GLASS, APPROX ST2E $36 \mathrm{MM}$ 10. 160 MM LGTH, CORNING 9480 OR KIMBLE 46170

Attachment B

UNIT PRICE STK PT CATALOG NO BUYER

\begin{tabular}{|c|c|c|c|}
\hline EA & .35 & 23 & $15-242-2473$ \\
\hline EA & .13 & 123 & $15-242-2478$ \\
\hline & .00 & 2 & $15-242-2493$ \\
\hline
\end{tabular}

$12.06 \quad 125 \quad 15-242-2608 \quad 36$

$\begin{array}{lllll}E A & 0.00 & 3 & 15-242-2613 & 36\end{array}$

EA $\quad 1.86 \quad 3 \quad 15-242-2624 \quad 36$

EA $13.98 \quad 3 \quad 15-242-2628 \quad 36$

EA $\quad .00 \quad 3 \quad 15-242-2630 \quad 36$

EA $\quad 3.01 \quad 3 \quad 15-242-2983 \quad 36$

EA $\quad 5.16 \quad 3 \quad 15-242-3003 \quad 36$

EA 3.84 $23 \quad 15-242-31103 \quad 10$

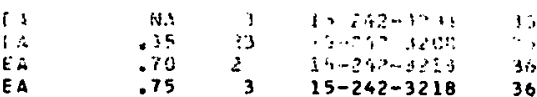

EA $\quad .47 \quad 12 \quad 15-242-3403 \quad 36$

EA $\quad 4.50 \quad 3 \quad 15-242-4003 \quad 36$

E $\quad 6.30 \quad 3 \quad 15-242-4008 \quad 30$

[A .10 2 19-232-US00 36

$\begin{array}{lllll}\text { EA } & 10.17 & 3 & 15-252-1010 & 36 \\ \text { EA } & 10.17 & 3 & 15-252-1020 & 36\end{array}$

EA $\quad .78 \quad 23 \quad 15-252-1110 \quad 36$

EA $\quad 1.11 \quad 3 \quad 15-252-1500 \quad 36$ 
Attachment B

UNIT PRICE STKPT CATALOG NO BUYER

TUBE. FILTER, BoROSILICATE GLASS, MITH SEALED IN FIBERGLAS FILTER DISC. ACE GLASS

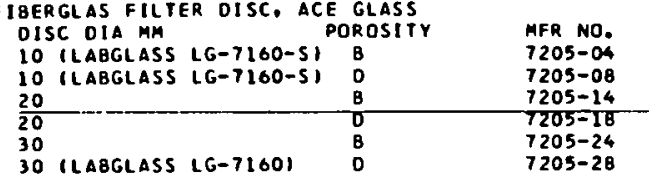

UBE, GAS COLLECTING, BOROSILICATE GLASS, WITH THO

STRAIGHT BORE CAPILLARY STOPCOCKS, SIT NO. 3 , APPROX SILE 5O MM IO. 310 MM LGTH, CAP. $250 \mathrm{ML}$, APPROX SILE SO HM IO. 3IO AM LGTH.

TUBE, GAS DISPERSION, BOROSILICATE GLASS, WITH 12 MM OD FRITTEO CYLINOER, 8 MM STEM DIA, $250 \mathrm{MM}$ MM OD FRITTED CYLINOER, 8 MM STEM DIA, 250 MM
LGTH. POROSITY C. CORNING 39533 CR KIMELE 28630

TUBE, GAS DISPERSION, BOROSILICATE GLASS, MITH

FRITIED DISC. OVERALL DIM ACROSS OISC AND STEM IS

$35 \mathrm{MH}$ FOR $20 \mathrm{MM}$ OISC, $45 \mathrm{MM}$ FOR $30 \mathrm{MM} \mathrm{CISC,} 6 \mathrm{MM}$

STEA DIA. 250 MM LGTH. CORNING 39525 - KIMBLE 28600

DISC DIA MM

20

30

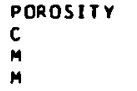

TUBE, MIXING, COMPARATOR, MARKET 5, 15 AND $17.5 \mathrm{ML}$. FOR USE WITH MIGH PHOSPHATE SLIDE COMPARATOR LAB TEST SET, CODE 1100. A TAYLOR 515. REPL PT

TUBE, REOUCTOR, JONES, LONC FORM, 27 IN. LGTH, $7 / 8$ IN. OD. FOR USF IN DFTERMINATION OF ION, MDLYBOENUM, VANADIUM ANO SIMILAR POL YVALENT ELEMENTS. NO. 39000, KIMBLE

TUBE, SAMPLE, MELTING POINT, CAPILLARY, THIN WALL GLASS CAPILLARIES TO HOLO SAMPLES, 1.5 TO 2 MM OD. 68 MM LGTH, 100 PCS IN GLASS VIAL. NO. 34500. KI MBLE

$\begin{array}{lllll}\text { EA } & 4.10 & 23 & 15-252-1550 & 36 \\ \text { EA } & 4.16 & 23 & 15-252-1555 & 36 \\ \text { EA } & 4.66 & 3 & 15-252-1560 & 36 \\ \text { EA } & 4.95 & 3 & 15-252-1565 & 36 \\ \text { EA } & 5.37 & 3 & 15-252-1570 & 36 \\ \text { EA } & 7.10 & 3 & 15-252-1575 & 36\end{array}$

TUBE, SEALING, BOROSILICATE GLASS, RECUCED ENDS, WITM FRITTED DISC, 90 MM OISC DIA, 25 MM TUBE DIA, 200 MM LGTH. CORNING 39580 OR KIMBLE 28720 POROSIIY

C

F

TUBE, TEST, CULTURE, WITH SCREW CAPS, $125 \mathrm{MM} \times 20$ MM. LUKNING 9825, KIMBLE 45OGQ OR APPROVCD EOUAL

TUBE. TEST, GRADUATEO FROM $5 / 10$ TO $10 \mathrm{ML}$ IN $1 / 10 \mathrm{ML}$ INCREMENTS, 16 MM OUTSIOE DIA. 125 MM LONG.
BOROSILICATE GLASS. WITH LIP, NO. 46350 . KIMBLE. BOROSILICAIE GLASS, WITH LIP, NO. 46350 , KIMBLE. OR APPROVED EOUAL

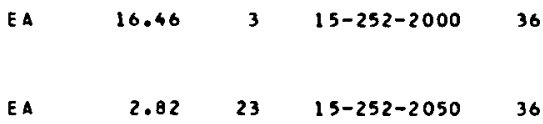

TUBE, IEST, BOROSILICATE GLASS, WITH RIM, NO, 9800 , CORNING OR KIMBLE $\$ 5042$

$\begin{array}{ll}\text { ORNING OR KIMBLE } 45042 & \text { LGTH MM } \\ \text { OD MM } & 100 \\ 13 & 125 \\ 15 & 150 \\ 16 & 150 \\ 18 & 150 \\ 20 & 150 \\ 25 & 200 \\ 25 & 250 \\ 25 & \end{array}$

TUBE, TEST, BOROSILICATE gLaSS, WITH RIM ANO SIDE $\triangle R M, 18$ MM OD, 150 MM LGTH. NO. 9840. CORNING OR KIMBLE 46225

TUBE, IEST, CULTURE, OISPOSABLE, TYPE PhaRMACEUTICAL GLASS, SIZE 10 MM OD, 75 MM LGTH, PALLET DACKEO, 5,, NAO PFR PALLET. NO. 73000, KIMBLE

TUBE, IEST, CULTURE, LIPLESS, 16 MH DO X 100 MH LGTH, NO. 45060, KIMBLE, OR APPROVED ECUAL

IURF, TFST, CULTURE, BOROSILICATE GLASS, WITHOUT RIM. CORNING 9820 OR KIMBLE 4504 Q

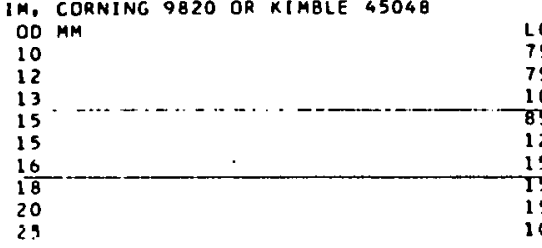

$\begin{array}{lccccc}\text { LGTH MM } & & & & & \\ 75 & \text { EA } & .05 & 23 & 15-255-5108 & 36 \\ 75 & \text { EA } & .06 & 12 & 15-255-5118 & 36 \\ 100 & \text { EA } & .05 & 23 & 15-255-5128 & 36 \\ 85 & \text { EA } & .05 & 3 & 15-255-5138 & 36 \\ 125 & \text { EA } & .06 & 2 & 15-255-5148 & 36 \\ 150 & \text { EA } & .07 & 2 & 15-255-5168 & 36 \\ 150 & \text { EA } & .07 & 23 & 15-255-5178 & 36 \\ 150 & \text { EA } & .11 & 23 & 15-255-5198 & 36 \\ 100 & \text { EA } & .10 & 3 & 15-259-5 ? 0 A & 36\end{array}$


Attachment B

UNIT PRICE STKPT CATALOG NO BUYER

TUBE, TEST. CULTURE, BOROSILICATE GLASS. WITHOUT RIM. CORNING $9820^{\circ}$ OR KIMBLE 45048 OD MM

25

LGTH MM - CONTINUFO-

200

TUBE, TEST, CULTURE, RESISTANCE GLASS, WITHOUT RIM, NO. K-45060, KIMBLE

OD MM

13

LGTH MM

150

TUBE, VACUUM COLLECTION, THERMAL RESI STANT GLASS, MUST BE DRY AND FREE FROM STRAIN, PYREX, UCC OWG CM-26138

TUBING, ASBESTOS, BRAIOED, COMMERC IAL GRAOE, 5 LB SPL. USED ON CLAMPS FOR HOLOING BEAKERS, JOHNSSPL. USED

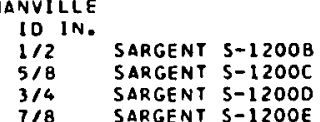

FT PER LB

FT PER LB
20
15
14
12

TUBING. CELLULOSE, SEAMLESS, CENCO 70160

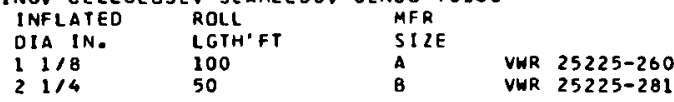

TUBING. CERAMIC. FLAT, SIZE 0.4 MM IO, 2.0 MM OO. 12 IN. LGTH, SO 1.10, FOURTH SERIES, CARBORUNDUM CO

TUB ING. FLUOROTHENE, KEL-F, $0.0375 \mathrm{IN}, 00 \times 0.062 \mathrm{IN}$ WALL THICKNESS. INSIDE ANO OUTSIOE SURFACES SMOOTH ANO FREE FROM DEFECTS, INSIDE DIA CONCENTRIC WITH OUTSIDE DIA. BORE O.251 IN. PLUS 0.000 IN. MINUS 0.010 iN.

TUB ING. FLUOROTHENE A, 1/4 IN, DO, CAT, NO. FNT2002. FABRICATEO BY UCC PLANT ORGDP

TUB ING. FLUOROTHENE A, 1/2 IN. OD, CAT. NO, FNT2004. FABRICATEO BY UCC PLANT ORGDP

TUB ING, GLASS, BOROSILICATE, I8 IN. LGTH, WITH SOUARE ENDS, CORNING OOB8 BRAND OR KIMBLE 46470 UD 5 WALL THK

TUB ING, KOVAR, 0.075 IN. 00, 0.010 IN. WALL THK IN ONE OR THO FT STRAIGHT LGTHS. NO. 900-75010. STIIPAKNFF

TUB ING, KOVAR, I IN, OD, 0.030 IN, HALL IHK, RANDOM LGTH. STUPAKOFF

TUBING, KOVAR, 0.125 IN. OD, 0.010 IN. WALL THK, NO. 90125010 , STUPAKOFF

TUBING, NYLON, 1000 PSI TESTEO BURSTING PRESSURE $1 / 2$ IN. OD. 0.380 IN. IO

TUB ING, POLYETHYLENE, 0.005 IN. THICK, COLOR TO BE BQIGHT RED. OPAOUE, 917-91B DENSITY. MELT INDEX BRIGHT REO, OPAOUE, 917-918 DEN
APPROX 2 OR LESS, 500 FT ROLL

APPROX 2 OR LESS
FLAT DIM. IN.

FLAT DIM.

3112

$$
9112
$$

TUBING, POLYETHYLENE, 0.005 IN. THK, 917-918 OENSITY, MELT INOEX APPROX 2 OR LESS, SOO FT ROLL. PPC NOUS TRIES

FLAT DIH. IN. CIRCUMFERENCE IN. 3

$91 / 2$

$131 / 4$

19 $26 \quad 1 / 2$

TU ING, POLYETHYLENE, LAY FLAT, 0.006 MIL THK, 48 IN. CIRCUMFERENCE, 24 IN. FLAT, CRYSTAL-X-CORP

TUB ING, POL YETHYLENE, LAY FLAT, 0.006 NIL TMK, 106

$\begin{array}{lrrrr}\text { EA } & .15 & 3 & 15-255-5218 & 36 \\ \text { EA } & .52 & 23 & 15-255-5238 & 36\end{array}$

$\begin{array}{lllll}E A & .03 & 3 & 15-255-5503 & 36 \\ \text { EA } & .04 & 3 & 15-255-5509 & 36\end{array}$

EA $\quad .94 \quad 2 \quad 15-258-6309 \quad 38$

$\begin{array}{lllll}\text { FT } & .00 & 3 & 15-261-1095 & 36 \\ \text { FT } & .00 & 3 & 15-261-1100 & 36 \\ \text { FT } & .00 & 3 & 15-261-1105 & 36 \\ \text { FT } & .00 & 3 & 15-261-1110 & 36\end{array}$

$\begin{array}{lllll}\text { ROLL } & 3.48 \quad 23 & 15-261-3100 \quad 36\end{array}$

EA $\quad .11 \quad 3 \quad 15-264-4000 \quad 36$

FI $\quad 2.54 \quad 5 \quad 15-270-3067 \quad 36$

FT $2.07 \quad 5 \quad 15-270-3127 \quad 36$

FI $\quad .91 \quad 3 \quad 15-270-3147 \quad 92$

$\begin{array}{lllll}\text { FT } & .01 & 3 & 15-273-3116 & 36 \\ \text { FT } & .02 & 3 & 15-273-3126 & 36 \\ \text { FT } & .05 & 3 & 15-273-3256 & 36\end{array}$

FI $.60 \quad 3 \quad 15-270-2100 \quad 36$

IN $\quad .44 \quad 5 \quad 15-276-2208 \quad 36$

F $1.13 \quad 3 \quad 15-276-2708 \quad 36$

FT $\quad .12 \quad 2 \quad 15-279-5010 \quad 92$

$\begin{array}{lrlll}\text { ROLL } & 9.45 & 3 & 15-282-1850 & 36 \\ \text { ROLL } & 25.65 & 3 & 15-282-1875 & 92\end{array}$

$\begin{array}{lllll}\text { FT } & .01 & 3 & 15-282-1980 & 92 \\ \text { FT } & .03 & 3 & 15-282-2000 & 92 \\ \text { FT } & .02 & 3 & 15-282-2010 & 92 \\ \text { FT } & .08 & 3 & 15-282-2020 & 92\end{array}$

FT $\quad .26 \quad 3 \quad 15-282-2030$

F T $\quad .20 \quad 3 \quad 15-282-2035 \quad 92$ 
Attachment B

TUBING, POLYETHYLENE, 0.006 TO 0.008 IN. THK, 917918 DENSITY, MELT INDEX APPROX 2 OR LESS, $500 \mathrm{FT}$ ROLL, PPC INDUSTRIES

$$
\text { FLAT DIM. }
$$

IN.

22
42

FERENCE IN

$\overline{84}$

TUBING, PLASTIC, BUNDLE, 7 TUBE, $1 / 4$ IN, POLYETHYLENE $1 / 16$ IN. POLYVINYL CMLORIDE SHEATH THO-LEAD TELE1116 IN. POLYVINYL CHLR INS SCOLOS - I BLUE, 2 RED. 2 BLACK AND 2 GREEN, PER ORNL INST OEPT SPEC IS-5-2 2 BLACK AND 2 GREE

TUBING, POLYETHYLENE, BUNOLE 10 TUBE, EA TUBE $1 / 4$ IN. OD, 1116 IN. BLACK POL YVINYL CHLORIDE SHEATH IN. OD, $1 / 16$ IN. BLACK POL YVINYL CHLORIDE SHEATH OVERALL. TWD-LEAD TELEPHONE WIRES INCLUDED IN ALL TUBES BLACK. EA TUBE NUMBER CODED AT 2 IN. INTERVALS. TUBES NUMBERED I THRU IO. WITH MYLAR SHEET BARRIER BETHEEN TUBE BUNDLE AND SHEATH, NO. PV-10-4. SAMUEL MOQRE

TUB ING, POLYETHYLENe, PURE, $1 / 4$ IN. OD TUBE, $X 0.040$ IN. HALL THK, COLOR TRANSLUCENT, ASTM 01248 , TYPE 1. CLASS B, GRADE 2, PLEXCO CO, NO SUB

TUB ING, POL YETHYLENE, PLASTIC, ASTM D 1248, TYPE 1. CLASS B, GRADE 2 , UNREPROCE SSED, NEUTRAL TRANSLUCENT COLOR, 1/16 IN. WALL THICKNESS OD IN.

1,4

$1 / 2$

318

TUB ING. POL YETHYLENE, NON-PLASTICIZED, FOR PACKING FINISHED FLUOROETHENE CORES, 0.0015 IN. THK, $91 / 2$ IN. FLAT LAY THK

TUBING, POLYETHYLENE, PLASTIC, ASTM D-1248, TYPE 1 , CLASS A. GRADE 4, UNREPROCESSED. NATURAL COLOR, $1 / 4$ CLASS A. GRADE 4. UNREPROCESSED NATUR OL COLOR, I/ INO. 4 -P IMPERIAL-EASTMAN. POL-4 EXTRCN CORP. OR NOP 44-P IMPERIA

TUB ING. POLYETHYLENE, PLASTIC, ASTM D-1248, TYPE II, CLASS $C$ OR B. UNREPROCESSED, TO FIT SWAGELOK STO CLASS C OR B, UNREPROCESSED, TO FIT

$1 / 4$ IN. 00. PLUS 0.005 IN.. MINUS 0.003 IN.. HALL PHICKNESS 0.060 IN.. PLUS OR MINLS 0.005 IN. COLOR ISOLID OR STRIPES ON BLACKI

COLOR

BI, UI:

GREEN

ORANGE

PURPL

REO

YELLOW

3/8 IN. OD, PLUS OR MINUS 0.005 IN.. WALL THICKNESS

0.062 IN.P PLUS OR MINUS 0.005 IN.

GOLOR ISOLIO ON STRIPES OH BLACKI

BLACK

BLUE

GREEN

RED

TUBING, POLYSTYRENE

$\begin{array}{lll}10 \text { IN. } & \text { HALL } & \text { LGTH } \\ 1 / 8 & \text { THK IN. } & \\ 1 / 4 & 1 / 16 & 6 \mathrm{FT} \\ 1 / 8 & 1 / 16 & 6 \mathrm{FT} \\ 318 & 1 / 16 & 6 \mathrm{FT} \\ 1 / 2 & 1 / 16 & 6 \mathrm{FT} \\ 578 & 1716 & 6 \mathrm{FT} \\ 3 / 4 & 1 / 8 & 6 \mathrm{FT} \\ 11314 & 1 / 8 & 6 \mathrm{FT} \\ 1314 & 1 / 8 & 6 \mathrm{FT}\end{array}$

TUBING, PYREX GLASS, CAPILLARY, 0.250 PLUS 0.000

INUS O.

MINUS O.OI G IN. OD. 2 HA PLUS OR MINU

TUBING, PYREX GLASS, 178 MA OD $X$ APPROX G FT LGTH $X$

5TD DALL FHICKNESS. NO. 234230 , CORNING

FT

FT

TUBING. PYREX Glass. CaPILlary TYPE, f F LGTH.

CORNING

BORE MH

$1 / 4$ TO $3 / 4$

$3 / 4$ TO 1114

OD

$610 \%$
MFR NO.

236510

236520

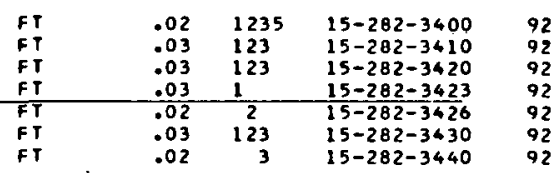

$\begin{array}{lrrrr}\text { FT } & .05 & 13 & 15-202-3500 & 92 \\ \text { FT } & .05 & 3 & 15-282-3505 & 92 \\ \text { FT } & .05 & 3 & 15-282-3510 & 92 \\ \text { FT } & .05 & 3 & 15-282-3520 & 92\end{array}$

92
2
2
2
2
2

2 
Attachment $B$

UNIT PRICE STK PT CATALOG NO BUYER

TUB ING, PYREX GLASS, CAPILLARY TYPE, \& FT LGTH. CORNING

BORE MM OO MM. MFR. NO. -CONTINU

$\begin{array}{lll}\text { BORE MM } & \text { OO MM. } & \text { MFR. NO. } \\ 1314 \text { TO } 2114 & 7 \text { TO } 8 & 236540 \\ 2114 \text { TO } 3 & 8 \text { TO } 10 & 236560 \\ & & \end{array}$

TUB ING. PYREX GLASS, COMBUSTION, FOR TEMP NOT TO
EXCEED 750 DEG C. OOI/2 IN.. HALL THK $3 / 32$ IN.. EXCEED 750 DEG C, OOI/2 IN.,
LGTH \& FT. NO. 11-370, F ISHER

TUBING, PYREX GLASS. HEAYY WALL, 3/8 IN. DO PLUS OR MINUS 0.023 IN.. WALL THK IN. S/O4, LGTH 48 IN.. MINUS 0.023 IN. WALL
CODE 237650 . CORNING

TUBING, PYREX GLASS, SPECIAL HALL, COOE 234104 , CORNING
OD MM
9
9

TUB ING, PYREX GLASS, MEO WALL, 48 IN. LGTM, CORNING

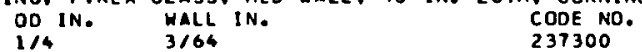
$\begin{array}{lll}1 / 4 & 3164 & 237300 \\ 1 / 2 & 1116 & 237310\end{array}$

TUBING, IPIPEI, GLASS, BOROSILICATE, PYREX 7740 OR $K I \operatorname{MAX} K G-33$ IO IN.

5 NON-PRECISION BORE, TEMPERED, STD WALL WITH STD 5 IN. CONICAL FLANGED ENDS, 45 IN. OVERALL.

2.005 PLUS OR MINUS 0.002 , PRECISION BORE, ANNEALED, MEAYY T, FLANGED ENDS, 10 OF FLANGE SECTION 0.062 iN. NINUS 0.000 , 24 IN. LGTH. FISHER AND PORTER

6 NON-PRECISION BORE, TEMPERED. STO WALL WITH STO $G$ IN. CONICAL FL ANGEO ENDS, 45 IN. OVERALL

-CONTINUED-

$\begin{array}{llllll}\text { FT } & \begin{array}{l}15 \\ \text { FT }\end{array} & \begin{array}{l}3 \\ .30\end{array} & 13 & \begin{array}{l}15-285-1136 \\ 15-285-1146\end{array} & 36 \\ \text { LB } & 1.28 & 1 & 15-285-3106 & 36 \\ \text { LB } & 1.94 & 1 & 15-285-4106 & 36 \\ & & & & \\ & & & & & \\ \text { FT } & .15 & 3 & 15-285-4200 & 36 \\ \text { LB } & 2.06 & 2 & 15-285-4206 & 36 \\ & & & & \\ \text { FT } & & & & \\ \text { FT } & .06 & 3 & 15-285-4216 & 36 \\ & .11 & 3 & 15-285-4218 & 36\end{array}$

OD VAR
MM MAL

THK MM LB/FT CORNING

\begin{tabular}{lllll}
4 & 0.3 & 0.8 & 0.012 & 234040 \\
5 & 0.3 & 0.8 & 0.016 & 234050 \\
6 & 0.3 & 1.0 & 0.023 & 234060 \\
7 & 0.3 & 1.0 & 0.028 & 234070 \\
\hline
\end{tabular}

$80.3 \quad 1.0$

io $0.4 \quad 1.4$

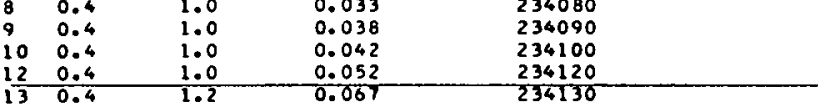

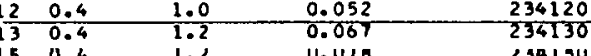

\begin{tabular}{lllll}
15 & 0.4 & 1.2 & 0.018 & 234130 \\
16 & 0.4 & 1.2 & 0.083 & 234160 \\
17 & 0.5 & 1.3 & 0.090 & 334170 \\
\hline
\end{tabular}

10.05

$\begin{array}{lll}20 & 0.6 & 1.2 \\ 22 & 0.6 & 1.5\end{array}$

$250.6 \quad 1.5$

$20.095 \quad 234180$

\begin{tabular}{lll}
5 & 0.145 & 234220 \\
5 & 0.166 & 234250 \\
\hline
\end{tabular}

$\begin{array}{lll}30 & 0.7 & 1 \\ 32 & 0.8 & 1\end{array}$

$35 \quad 0.8$

3.0

$451.0 \quad 2.0$

$\frac{51 \quad 1.0}{54} 1.0 \quad 2.0$

601.1

$641.2 \quad 2.4 \quad 0.66 \quad 0.69$

$\begin{array}{llll}1.2 & 2.4 & 0.66 & 234640 \\ 1.4 & 0.75 & 234700\end{array}$

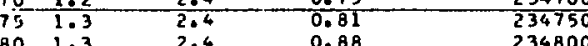

$\begin{array}{lllll}80 & 1.3 & 2.4 & 0.88 & 234800 \\ 90 & 1.3 & 2.4 & 1.00 & 234900\end{array}$

$\begin{array}{llll}1001.4 & 2.4 & 1.112 & 2349000\end{array}$

TUBING. PYREX GLASS, STD NALL, CORAING

OD MM

110
120
125
130
140
150

CORNING CODE

TURING, PYREX GLASS, STO WALL, 4 FT LGTH, CORNING

$\begin{array}{lll}234310 & F T & 2.56 \\ 234330 & F T & 3.43\end{array}$

$234340 \ldots \ldots$ FT $\quad 3.15$

$234360^{-\cdots} \cdots \ldots+F T-\cdots, \frac{3}{3.68}$

234370
234390

EA

$106.80 \quad 2 \quad 15-285-4256 \quad 97$

EA $\quad 35.46 \quad 3 \quad 15-285-4271 \quad 97$

PC $\quad 105.40 \quad 2 \quad 15-285-4298 \quad 97$

$\begin{array}{lllll} & & \text { WALL } & & \\ \text { OD } & \text { VAR } & \text { THK } & & \text { MFR } \\ \text { MM } & \text { MM } & \text { MM } & \text { LB/FT } & \text { NO. } \\ 4 & 0.3 & 0.8 & 0.012 & 234040 \\ 5 & 0.3 & 0.8 & 0.016 & 234050\end{array}$

234040

FT

$.023 \quad 15-285-651$

$15-285-6516 \quad 36$ 
Attachment $\dot{B}$

UNIT PRICE STK PT CATALOG NO BUYER

TUBING, PYREX GLASS, STO WALL, 4 FT LGTH, CORNING

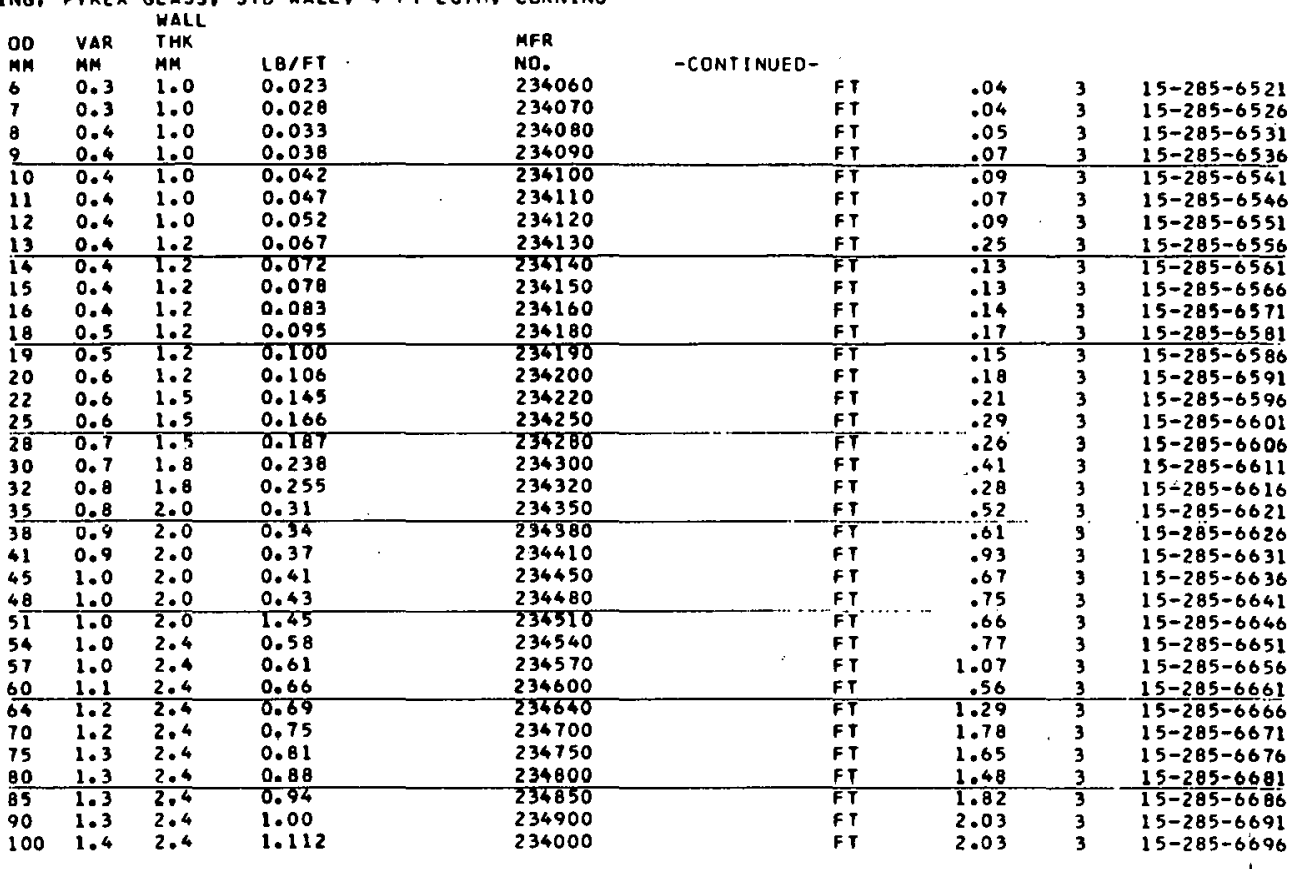

TUBING. PYREX GLASS, FLANGEO BOTH ENOS, FISCHER AND PORTER

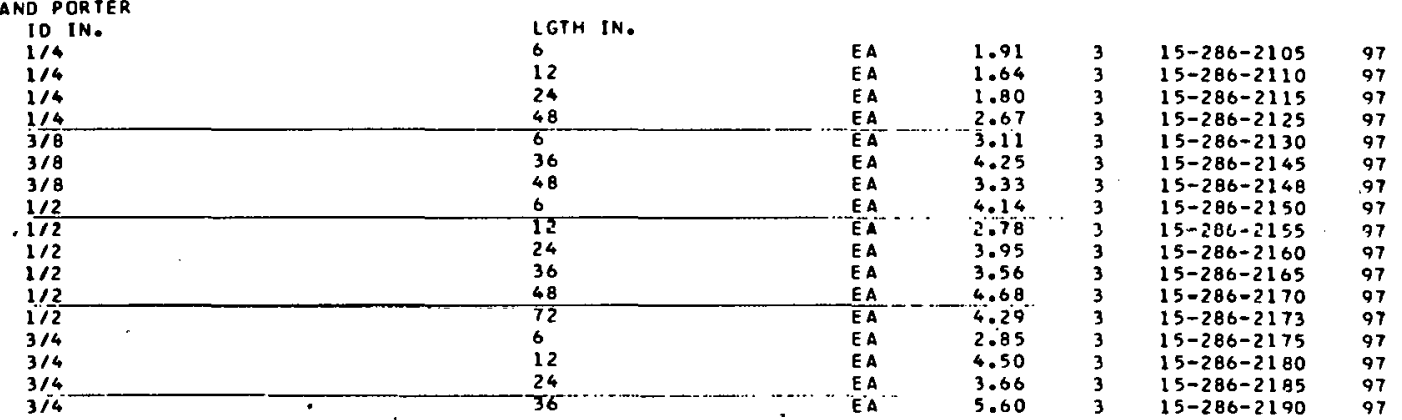

TUBING, PYREX GLASS, hEAVY HALL, WITHOUT FLANGES, CORNING

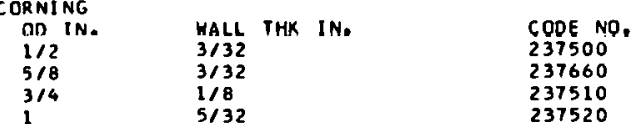

TUB ING, (PIPE), GLASS, BOROSILICATE, PYREX 7740 OR $K$ IMAX KG-33, CONICAL FLANGED ENDS, $11 / 2$ IN. 10 $127 / 32$ IN. OD. LGTH 48 IN., CODE 72-7130

QDE NQ. 37660 37520

TUBING, IPIPE), GLASS, BOROSILICATE, PYREX 7740 OR KIMAX KG-33. STO HALL. TEMPERED, CONICAL FLANGED ENDS

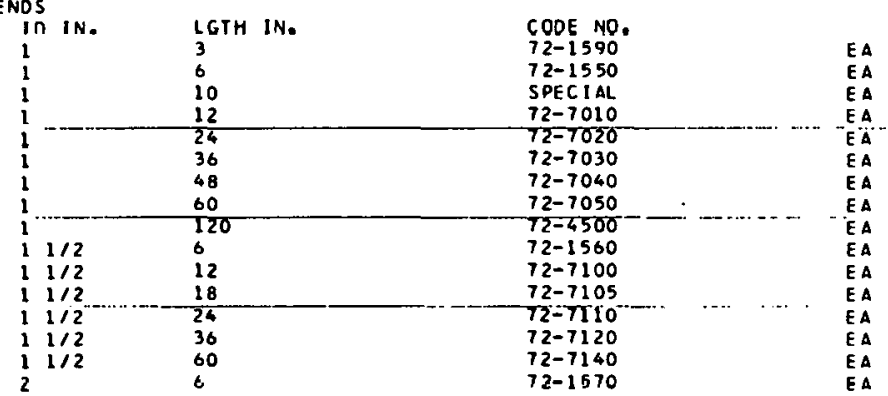

$\begin{array}{lllll}\text { FT } & .42 & 23 & 15-286-3105 & 97 \\ \text { FT } & .05 & 2 & 15-286-3110 & 97 \\ \text { FT } & .85 & 3 & 15-286-3115 & 97 \\ \text { FT } & .78 & 3 & 15-286-3120 & 97\end{array}$


Attachment B

UNIT PRICE STK PT CATALOG NO BUYER

TUB ING, IPIPEI, GLASS, BOROSILICATE, PYREX 7740 OR KIMAX KG-33, STD HALL. TEMPERED, CONICAL FLANGED KIMAX

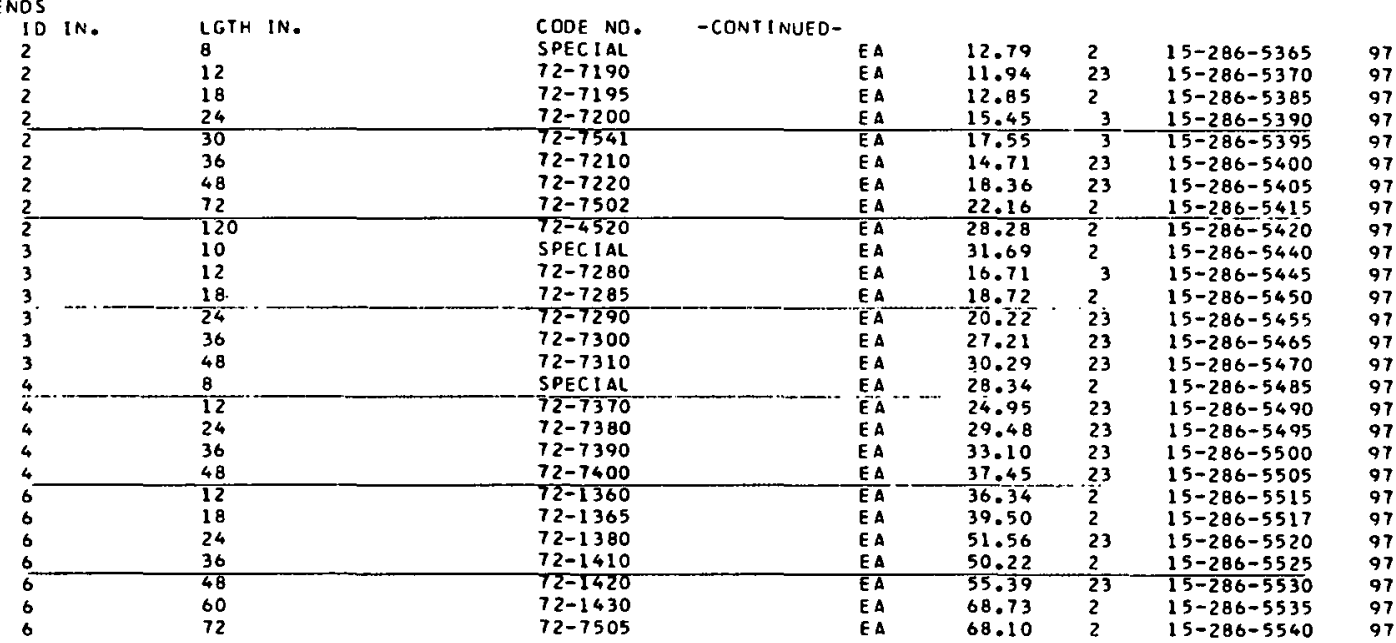

TUBING, OUARTL, CLEAR FUSED, HEAYY WALL, 99.8 PER

CENT SILICON DIOXIDE MIN. 2.0 MM WALL THICKNESS,

$4 O$ IN. LCTHS, AMERSIL OUARTZ DIV. ENGELHAPD

INDUSTRIES INC

ID MM

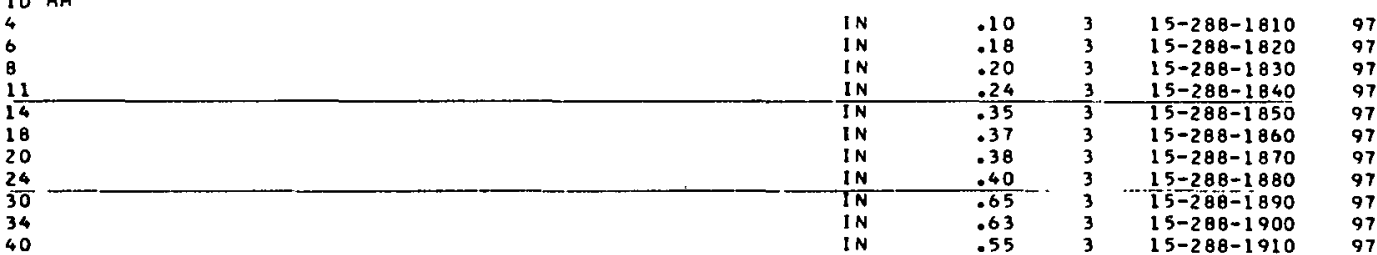

TUBING, OUARTZ, FUSED, CLEAR, 48 IN. LGTH, Y-MS-I2I

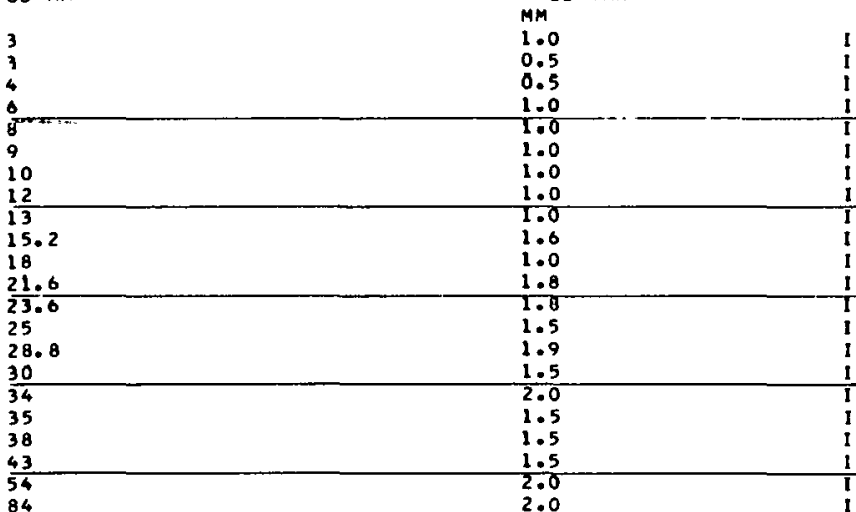

TUBING, QUART2, FUSED, TRANSPARENT, 2 IN. $10 \times 1.75$

MK WALL $X 30$ IN. LGTH, Y-MS-121

TUBING, QUARTZ, FUSED, TRANSPARENT, 0.056 IN. DO PLUS

DR MINUS 8 PER CENT, 0.025 IN. IO PLUS OR MINUS

0.005 IN. IN RANDOM LGTHS OF NOT LESS THAN 6 IN.

GE, $Y-M S-121$

TUBING, OUART2, FUSED, TRANSPARENT, SINGLE BORE, 24

IN. LGTH, 2.0 ID MM, 8.0 OD MM, NO. S, GE, Y-MS-12

TUBING, OUART2, FUSED, TRANSPARENT, 0.5 TO $1.0 \mathrm{MM}$

$10 \times 5.5$ TO 6.5 MH OD $\times$ IO IN. LGTH, Y-MS-121

TUBING, OUARTZ, FUSEO, TRANSPARENT, DPEN ENDS, IN.

OD $\times 1 / 8$ IN. WALL $X 24$ IN. LGTH, WALL THICKNESS

CRITICAL. GE, $Y-M S-121$

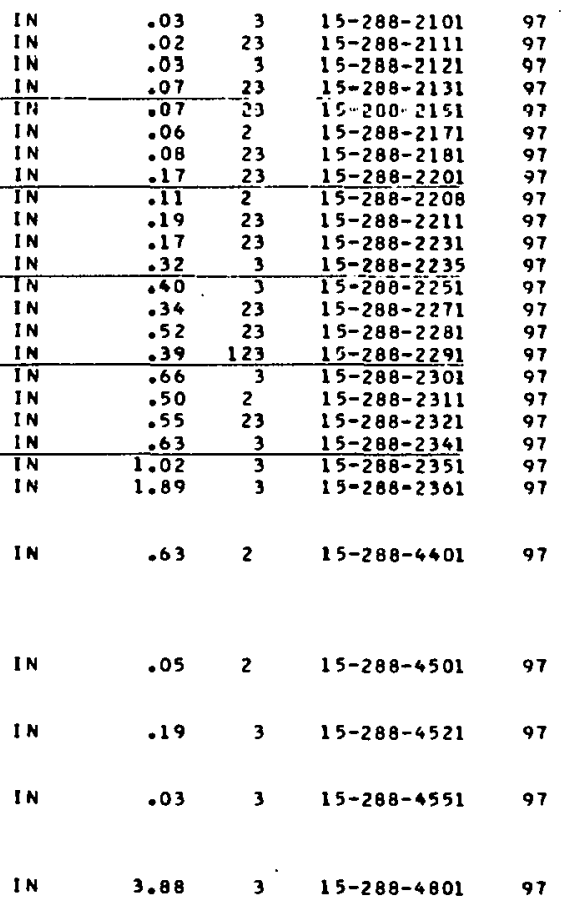


Attachment B

TUBING, OUARTZ, FUSED. TRANSPARENT, WITH TEST TUBE END, 2112 IN. ID $\times 1.75 \mathrm{Mm}$ WALL $\times 30$ IN. LGTH. $E N D, 2,212$
$Y \rightarrow-121$

TUB ING, RUBBER, BLACK, INSERTED FABRIC REINFORCED,

I/4 IN. ID. WALL THK IIB IN.. WILL VWR 56435-I87

TUB ING, RUBBER, BUNA

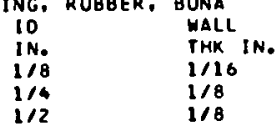

METILER 300

METILER 300

METTLER 300

TUS ING. RUBBER, NEOPRENE

10 IN.

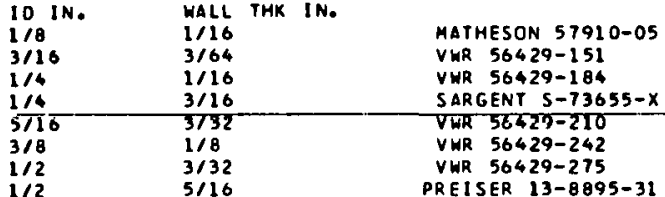

UBING, RUBBER, PRESSURE AND VACUUM, RED, HIGH QUALIIY REO RUBBER TUBING FOR PRESSURE AND

VACUUM. FLEXIBLE, 1/4 IN. ID, WALL IHK 3/16 IN.

PREISER 13-8080-12 OR VER 56433-239

TUBING, RUBBER, PRESSURE AND YACUUM, BLACK, HIGH QUALI IY RUBBER. FLEXIBLE, 3/8 IN. 10, 1/4 IN. WALL, OUALITY RUBBER, FLEXIBLE, 3/

TUBING, RUBBER, PRESSURE ANO VACUUM, GLACK WITH HEAYY WALL I/2 IN. ID, WALL THK I/4 IN. NO. HEAYY WALL. 1/2 IN. ID, WALL THK $1 / 4$

TUB ING. RUBBER, LATEX, AMBER, transluCENT, MS 15-ACIIA

LIGHT WALL

ID IN.

$1 / 8$

118 .

$3 / 16$

$5 / 16$
$3 / 8$

$1 / 2$

WALL THK

IN.

$1 / 32$

$3 / 64$
$1 / 16$

$1 / 16$

HEAVY HALL

$1 / 16$
$3 / 32$

\begin{tabular}{|c|c|c|c|c|}
\hline EA & 35.00 & 23 & $15-288-5501$ & 97 \\
\hline FT & .00 & 1 & $15-291-1112$ & 36 \\
\hline
\end{tabular}

$10 \mathrm{IN}$.

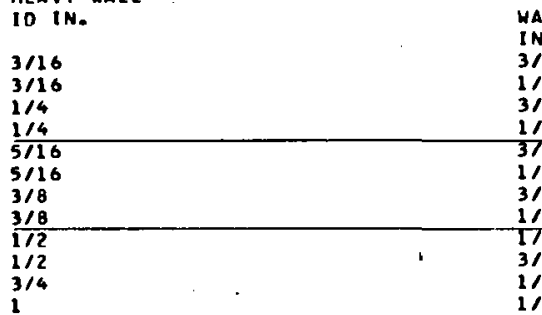

HALL THK

N.

$\begin{array}{lllll}3132 & \text { FT } & .09 & 1235 & 15-291-6137\end{array}$

$\begin{array}{lllll}18 & \text { FT } & .13 & 1235 & 15-291-6142\end{array}$

$.16 \quad 1235 \quad 15-291-6162$

$.191515-291-6187$

$\begin{array}{llll}.16 & 123 & 15-291-6207\end{array}$

$1 / 82$

178

PRESSURE

PRESSURE

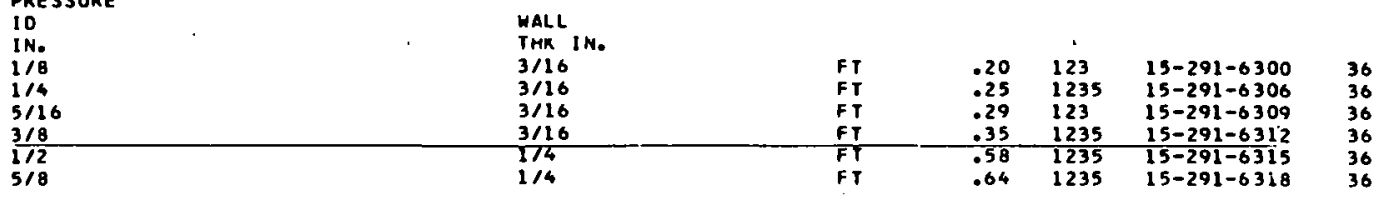

TUBING. RUBBER, PURE GUM, AMBER, SUITABLE FOR HIGH VACUUA, SIIE 3/4 IN. IO X $3 / \theta$ IN. WALL. SPEC $Z l=$ Y-8318. FILLOIIER SURGICAL SUPPLY CO

TUBING, RUBBER, PURE GUM, AMBER, SUITABLE FOR HIGH VACUUM. SIZE I IN. ID $X 3 / 8$ IN. HALL. SPEC 2Z-T318. FILLAUER SURGICAL SUPPLY Co

TUBing, RUBBer, PURE GUM, GOOCH STYLE, tRansluCENT

ID IN.

5/8 PREISER 13-9080/03

PSIU 14-1BOA
FLAT WIOTH

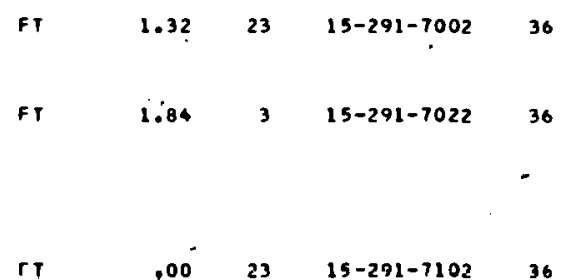


Attachment B

UNIT PRICE STK PT CATALOG ND BUYER

TUBING, PLASTIC. TRANSPARENT, TENSIL STRENGTH, 3000

LB/SO IN.. DIELECTRIC STRENGTHO DRY 1000 VOLTIMIL,

WET 800 VOLTIMIL. WATER ABSORPTICNO O.4 PER CENT

IN WEIGHT AFTER 24 HRS IMMERSION, OPERATING TEMP

UP TO 1 IOO DEG F, SULFLEX OR MMM CO

$\begin{array}{lll}10 \text { IN. } & \text { HALL THK IN. OLE NO. TONTINUED- } \\ 1.000 & 0.035 & \text { SIIN. }\end{array}$

HiNG, PLASTIC, TRANSPARENT TRANSFLEX, iRVINGTON

VARNISH AND INSULATOR CO

ARNISH AND INSULATOR CO

\begin{tabular}{|c|c|c|c|c|c|c|c|c|}
\hline $\begin{array}{l}\text { AHG } \\
10 \\
4 \\
2\end{array}$ & $\begin{array}{l}\text { MAX } \\
0.112 \\
0.224 \\
0.278\end{array}$ & $\begin{array}{l}\text { MIN } \\
0.102 \\
0.204 \\
0.258\end{array}$ & $\begin{array}{l}\text { NOM } \\
0.106 \\
0.208 \\
0.263\end{array}$ & $\begin{array}{l}F T \\
F T \\
F T\end{array}$ & $\begin{array}{l}.03 \\
.05 \\
.06\end{array}$ & $\begin{array}{l}1 \\
1 \\
1\end{array}$ & $\begin{array}{l}15-303-9000 \\
15-303-9030 \\
15-303-9040\end{array}$ & $\begin{array}{l}92 \\
92 \\
92\end{array}$ \\
\hline
\end{tabular}

TUBING, NON-RIGIO, POLYYINYL CHLORIOE, FOR GENERAL LABORATORY USE. MS 15-AC-16A

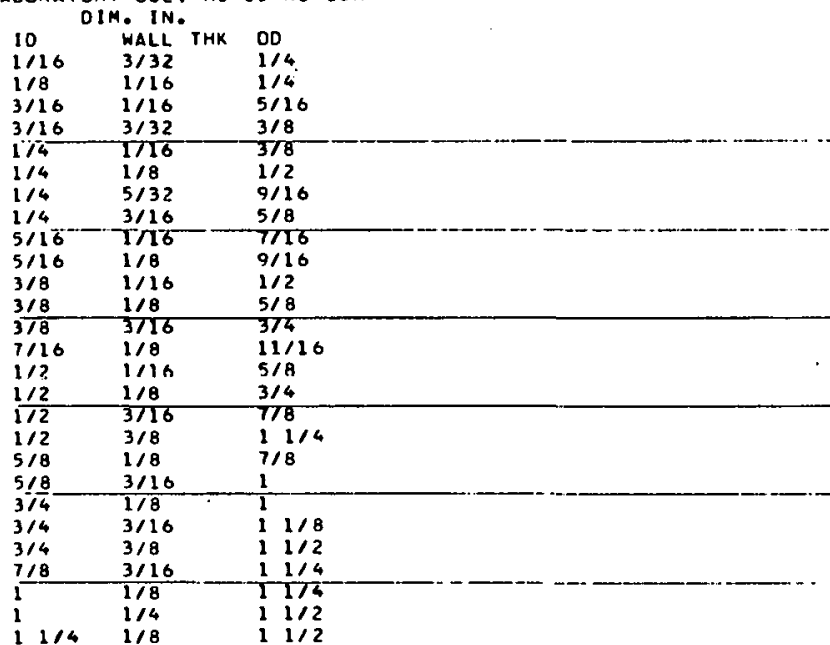

\begin{tabular}{|c|c|c|c|}
\hline $\begin{array}{l}F T \\
F T\end{array}$ & .02 & $\begin{array}{r}23 \\
123\end{array}$ & $\begin{array}{l}15-306-2100 \\
15-306-2110\end{array}$ \\
\hline $\begin{array}{l}\text { FT } \\
\text { FT }\end{array}$ & .02 & $\begin{array}{r}123 \\
23\end{array}$ & $\begin{array}{l}15-306-2110 \\
15-306-2120\end{array}$ \\
\hline FT & .04 & 23 & $15-306-2130$ \\
\hline & .03 & 1235 & $15-308-2140$ \\
\hline FT & .06 & 125 & $15-306-2142$ \\
\hline FT & .07 & 23 & $15-306-2150$ \\
\hline F T & .09 & 23 & $15-306-2160$ \\
\hline FT" & -.03 & 23 & $15-306-2170$ \\
\hline F T & .06 & 1 & $15-306-2172$ \\
\hline FT & .04 & 23 & $15-306-2180$ \\
\hline FT & .08 & 123 & $15-306-2190$ \\
\hline FT & .23 & 5 & $15-306-2192$ \\
\hline FT & .09 & & $15-306-2194$ \\
\hline FT & .05 & 1235 & $15-306-2200$ \\
\hline $\mathbf{F} \mathbf{T}$ & .11 & 23 & $15-306-2210$ \\
\hline FT & .19 & 25 & $15-306-2212$ \\
\hline FT & .81 & 15 & $15-306-2220$ \\
\hline FT & .12 & 23 & $15-306-2230$ \\
\hline $\mathbf{F T}$ & $\therefore 8$ & 23 & $15-306-2240$ \\
\hline$F T$ & .14 & 1235 & $15-306-2250$ \\
\hline F T & .39 & 5 & $15=306-2252$ \\
\hline F T & .61 & 2 & $15-306-2255$ \\
\hline FT & .30. & 23 & $15-306-2260$ \\
\hline FT & $.19^{\circ}$ & 123 & $15-306-2270$ \\
\hline FT & .46 & 1 & $15-306-2272$ \\
\hline & & & $15-306-2280$ \\
\hline
\end{tabular}

TUBING. TYGON, CRYSTAL CLEAR, 5/16 IN. IC $X 1 / 8$ IN.

WALL THK $X 9 / 16$ IN. DD, FORMULATION B44-3, US

STONE WARE

TUEING, VYCOR, STO WALL, 36 IN. LGTH, CORNING

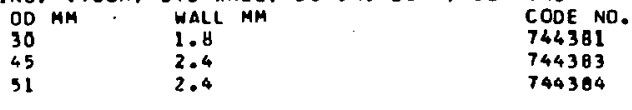

F T

$15-306-2280$

92
92
92
92
92
92
92
92
92
92
92
92
92
92
92
92
92
92
92
92
92
92
92
92
92
92
92

-IUBE_EUIINGSL_BOBOSHISATE_GLASS

$\begin{array}{lllll}P C & 11.96 & 3 & 15-310-5000 & 36 \\ P C & 10.77 & 3 & 15-310-5020 & 36 \\ P C & 30.74 & 3 & 15-310-5040 & 36\end{array}$

ADAPTOR. INTER-JOINT, BOROSILICATE GLASS. NO. J-205. SGA

\begin{tabular}{lll} 
& \multicolumn{2}{c}{ S/T JOINT NO. } \\
SIZE & FEMALE & MALE \\
AB & $12 / 30$ & $45 / 50$ \\
$B D$ & $14 / 35$ & $19 / 38$ \\
$B R$ & $19 / 38$ & $14 / 35$ \\
CH & $19 / 30$ & $55 / 50$ \\
CM & $24 / 40$ & $10 / 30$ \\
$C O$ & $24 / 40$ & $19 / 38$ \\
$C P$ & $24 / 40$ & $24 / 40$ \\
DL & $29 / 42$ & $19 / 38$ \\
OH & $29 / 42$ & $24 / 40$ \\
EB & $34 / 49$ & $19 / 38$ \\
EC & $34 / 45$ & $24 / 40$
\end{tabular}

LABGLASS

- $1190-C-6$ LG- $1190-0-1$ LG-1190-E-7 $G-1200-8-1$ L G- $1200-\mathrm{B}-6$ $1 G-1200-\mathrm{C}-3$ EA 3.21 $G=1200-C-4 \quad \cdots \quad 3.06$ $L G=1200-0-1$

ADAPTOR, STOPCOCK, BOROSILICATE GLASS, LAPINE

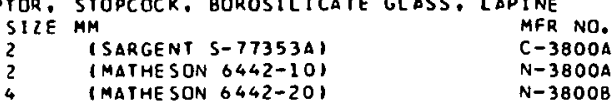

ADAPIOR, TUBE, BOROSILICATE GLASS, 105 OEG ANGLE, $24 / 40$ S/T JOINT. CORNING 8840 OR KIMBLE 10010

ADAPTOR. TUBE, REDUCING, BOROSILICATE GLASS,NO, 8820. CORNING

$$
\begin{array}{ll}
\text { SIT JOINT NO. } \\
\text { FEMALE } & \text { MALE } \\
10 / 30 & 19 / 30 \\
10 / 30 & 24 / 40 \\
14 / 35 & 24140 \\
19 / 38 & 24 / 40
\end{array}
$$

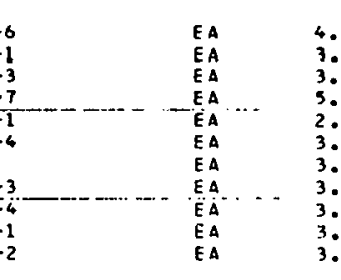

4.35
3.03
3.03
3.27
2.72
3.19
3.21
3.06
3.55
3.54
3.06

15-315-1607 $15-319-1617$ 15-315-1627 $13-315-1637$
$15-315-1647$ $15-315-1647$
$15-315-1657$ $15-315-1667$ 15-315-1677 $15-315-1687$ $15-315-1697$
$15-315-1707$ 2 
Attachment B

IUBE_EIIIIUGS__BOBOSIUICAIE_GLASS

UNIT PRICE STK PT CATALOG NO BUYER

ADAPTOR. TUBE, REDUCING, BOROSILICATE GLASS,NO. B820. CORNING

SIT JOINT NO.

FEMALE MALE

$19138 \quad 29142$

$19138 \quad 45 / 50$

$24140 \quad 29142$

$\begin{array}{ll}24 / 40 & 34 / 45 \\ 24 / 40 & 45 / 50\end{array}$

-CONTINUED

$\begin{array}{lrrrr}\text { EA } & 4.25 & 3 & 15-315-5197 & 36 \\ \text { EA } & 5.98 & 23 & 15-315-5217 & 36 \\ \text { EA } & 3.69 & 3 & 15-315-5227 & 36 \\ \text { EA } & 4.15 & 3 & 15-315-5237 & 36 \\ \text { EA } & 5.31 & 3 & 15-315-5247 & 36\end{array}$

ADAPTOR, TUBE, REDUCING, BOROSILICATE GLASS, BUSHING

TYPE, NO. 8825, CORNING

SIT JOINT NO.

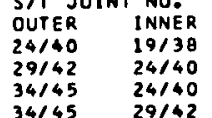

$\begin{array}{lrrrr}\text { EA } & 2.46 & 23 & 15-315-5607 & 36 \\ \text { EA } & 3.07 & 23 & 15-315-5617 & 36 \\ \text { EA } & 3.53 & 3 & 15-315-5627 & 36 \\ \text { EA } & 3.33 & 3 & 15-315-5637 & 36\end{array}$

JOINT, GROUND, BALL AND SOCKET, BALL ONLY, BORO-

SILICATE GLASS, CORNING 6762 DR KIMELE 33602

SIT NO.

$12 / 1$
$12 / 1$

1212

$12 \frac{13}{15}$

$18 / 7$

$20 / 12$

$35 / 20$

$35 / 25$

$60 / 30$

$75 / 50$

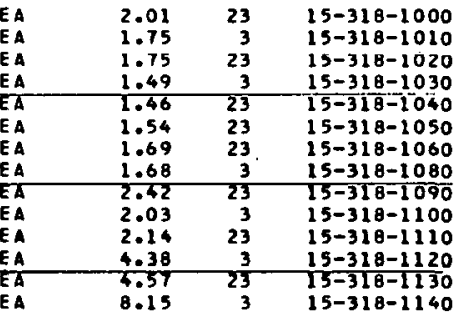

36
36
36
36
36
36
36
36
36
36
36
36
36
36

JOINT, GROUND, BALL ANO SOCKET, SOCKET ONLY, BORO-

SILICATE GLASS. FEMALE, CORNING 6764 OR KIMBLE

33604

$5 / T$ NO.

1211

121112

1212

1213

$18 / 7$

1819

$28 / 12$

$35 / 20$

35125

50130

79190

JOINT, GROUND. FULL LGTH. GOROSILICATE GLASS, SIT

INTERCHANGEABLE, S/T NO. 103/60, 100 MN OO STRAIGHT

TUBIMG. NO. 75h5. ACE GLASS

PART
QUTER ILABGLASS LG-1001)

EA

$10.302 \quad 15-318-1980$

36

JOINT, GROUND, FULL LGTH, INNER PT ONLY, BOROSILICATE

GLASS, S/T INTERCHANGFABIE, MAI.E, C.NBNING G560.

SIABLE 33502

TITELE 33 SO

$5 / T N D$

$7 / 25$

$10 / 30$

$12 / 30$
$14 / 35$

19138

24140

$29 / 42$

$\frac{34 / 45}{40 / 50}$

45150

60/50

$55 / 50$

$71 / 60$

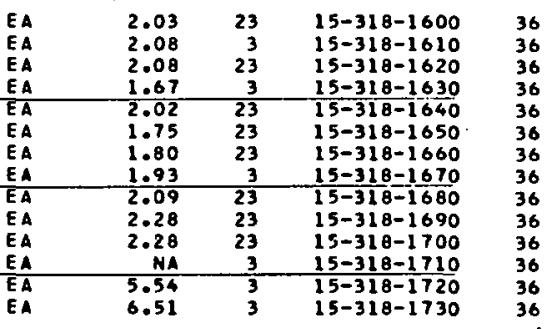

JOINT, GROUND, FULL LGTH, OUTER PART ONLY, BORO-

SILICATE GLASS, S/T INTERCHANGEABLE. FEMALE, NO.

6580. CORMING OR NO. 33310, KIMBLE

S/T NO.

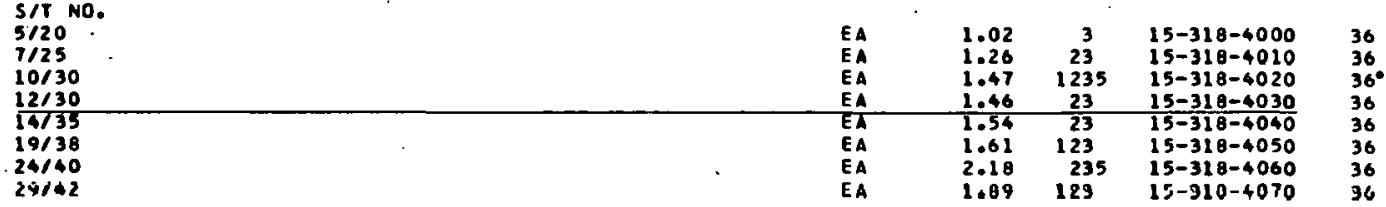


Attachment B

_UUBE_EIIILGSE_BOBOSLLLAIE_GLASS -

UNIT PRICE STK PT CATALOG NO BUYER

JOINT, GROUND, FULL LGTH, DUTER PART ONLY, GORO-

SIL ICATE GLASS. SIT INTERCHANGEABLE. FEMALE, NO. 6580 , CORNING OR NO. 33510 , KIMBLE

6580, CORNIN

$34 / 45$

$40 / 50$
$45 / 50$

$50 / 50$

55150

60150
$71 / 60$

JOINT, GROUND, MED LGTH, INNER PT CNLY, BOROSILICATE

GLASS, SIT INTERCHANGEABLE, CORNING 6620 OR KIMBLE

33562

SIT NO.

$5 / 12$

10118

$\frac{19122}{12118}$

14120

JOINT. GROUNO, MED LGTH, OUTER PT CNLY, EOROSILICATE

GLASS, S/T INTERCHANGEABLE, CORNING 6640 OR KIMBLE

33564

S/T NO.

$5 / 12$

5112

10118

12118

$14 / 20$
$19 / 22$

-CONTINUFD-

$\begin{array}{lllll}\text { EA } & 2.60 & 23 & 15-318-4080 & 36 \\ \text { EA } & 3.08 & 23 & 15-318-4090 & 36 \\ \text { EA } & 3.77 & 23 & 15-318-4100 & 36 \\ \text { EA } & 4.04 & 3 & 15-318-4110 & 36 \\ \text { EA } & 4.44 & 23 & 15-318-4120 & 36 \\ \text { EA } & 5.03 & 23 & 15-318-4130 & 36 \\ \text { EA } & 7.20 & 23 & 15-318-4140 & 36\end{array}$

JOINT, INTER-JOINT, CAPILLARY, BOROSILICATE GLASS

$\begin{array}{lllll} & \text { IO } & \text { SIT JOINT } & \text { SGA } & \text { LABGLASS } \\ \text { TYPE } & \text { MM } & \text { NO. } & \text { NO. } & \text { NO. } \\ \text { MALE } & 1.0 & 10 / 30 & J-160 & \text { LG-1150-3 } \\ \text { MALE } & 1.5 & 10 / 30 & J-160 & \text { LG-1150-4 } \\ \text { MALE } & 2.0 & 10 / 30 & J-160 & \text { LG-1150-5 }\end{array}$

JOINT, INTER-JOINT, BOROSILICATE, MERCURY SEAL, SIT $7 / 25$, SGA J-175

JOINT, INTER-JOINT, MITH REDUCED TUBE, BOROSILICATE GLASS, LABGLASS NO. LG-1170
SII NO.
TUBE OD MM

$7 / 25$

$10 / 30$
$12 / 30$

12/30 (ACE 7630-06)

$14 / 35$

19/36 IACE 7630-101

$24 / 40$

$29 / 42$
$34 / 45$

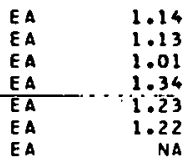

1.13

1.01

$\begin{array}{rr}3 & 15-318-4580 \\ 3 & 15-318-4590 \\ 3 & 15-318-4600 \\ 3 & 15-318-4610 \\ 3 & 15-318-4620 \\ 23 & 15-318-4630 \\ 3 & 15-318-4640\end{array}$

36
36
36
36
36
36
36

JOINT, O-RING, DLAIN, CORNING 6780 OR KIMBLE 33650

APPROX CLAMPSILE

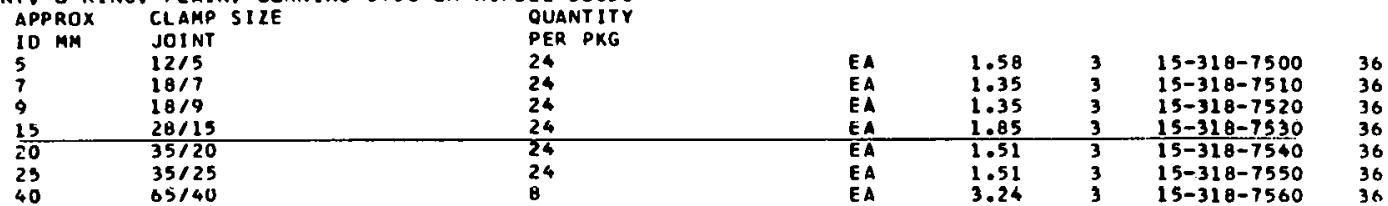

JOINT, SEMI-BALL, BOROSILICATE GLASS, 6 IN, LGTH

\begin{tabular}{|c|c|c|c|c|c|c|c|c|c|c|}
\hline $\begin{array}{l}\text { TYPE } \\
\text { I NNER } \\
\text { I NNER }\end{array}$ & $\begin{array}{l}\text { BALL } \\
\text { BALL }\end{array}$ & $\begin{array}{l}\text { SEAI } \\
\text { BALL } \\
\text { SIZE } \\
40 / 25 \\
102175\end{array}$ & $\begin{array}{l}\text { BALL } \\
\text { OIA } \\
\text { MM } \\
40 \\
10\end{array}$ & $\begin{array}{l}\text { TUBE } \\
10 \\
\text { MH } \\
25 \\
75\end{array}$ & $\begin{array}{l}S G A \\
N O A \\
J J-1275 \\
J J-1275\end{array}$ & $\begin{array}{l}\text { LABGLASS } \\
\text { NO. } \\
\text { LG-1040 } \\
\text { LG-1040 }\end{array}$ & $\begin{array}{l}\text { EA } \\
\text { EA }\end{array}$ & $\begin{array}{r}1.95 \\
10.95\end{array}$ & $\begin{array}{l}3 \\
3\end{array}$ & $\begin{array}{l}15-318-7600 \\
15-318-7620\end{array}$ \\
\hline CUTER & SOCKET & $28 / 11$ & 28 & 11 & $\begin{array}{l}J J-1275 \\
\text { IACE NO. }\end{array}$ & $\begin{array}{c}\text { LG-1041 } \\
7655-301\end{array}$ & EA & 1.53 & 3 & $15-318-7640$ \\
\hline $\begin{array}{l}\text { OUTER } \\
\text { OUTER }\end{array}$ & $\begin{array}{l}\text { SOCKET } \\
\text { SOCKET }\end{array}$ & $\begin{array}{l}40 / 25 \\
102 / 75\end{array}$ & $\begin{array}{l}40 \\
10\end{array}$ & $\begin{array}{l}25 \\
75\end{array}$ & $\begin{array}{l}J J-1275 \\
J J-1275\end{array}$ & $\begin{array}{l}L G-1041 \\
L G-1041\end{array}$ & $\begin{array}{l}E A \\
E A\end{array}$ & $\begin{array}{r}2.79 \\
10.26\end{array}$ & $\begin{array}{l}3 \\
3\end{array}$ & $\begin{array}{l}15-318-7600 \\
15-318-7600\end{array}$ \\
\hline
\end{tabular}

SEAL. D-RINGS ONLY, BUNA N, MIN QUANTITY 12 , CORNING

G780 OR KIMBLE 33652

780 OR
SIIE

15-110

$15-11$

$\begin{array}{r}15-112 \\ 15-116 \\ \hline\end{array}$

$15-214$

$15-124$

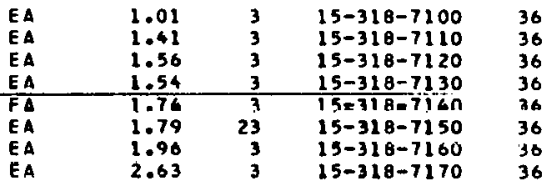

$\begin{array}{lllll}\text { EA } & .12 & 3 & 15-320-1500 & 34 \\ \text { EA } & .12 & 3 & 15-320-1510 & 36 \\ \text { EA } & .13 & 3 & 15-320-1520 & 36 \\ \text { EA } & .64 & 3 & 15-320-1530 & 36 \\ \text { EA } & .17 & 3 & 15-320-1540 & 36 \\ \text { EA } & .16 & 3 & 15-320-1550 & 36 \\ \text { EA } & .17 & 3 & 15-320-1560 & 36\end{array}$


IUAE ELIUHGS EOR POROSULICATE GLSS PIPE

AOAPTOR, BUTTON, NICKEL PLATED STEEL, FOR BOROSILICATE GLASS PIPE, CODE 72-9651

COUPL ING, GLASS TO GLASS, 24 ST ALUMINUM, BOROSILICATE GLASS, FISCHER AND PORTER

IO IN.

11

COUPL ING. METAL TO GLASS, 24 ST ALUMINUM, 303 STAIMLESS STEEL. BOROSILICATE GLASS. FISCHER AND PORTER

ID IN.

$1 / 4$

$3 / 4$

ELBOW, PIPE, GLASS, BOROSILICATE, PYREX 7740 OR $K I$ MAX KG-33, 1/2 IN. I0, 90 DEG, TEAPEREO

ELBOH. PIPE, GLASS, BOROSILICATE TYPE, PYREX 7740 OR KIMAX KG-33, TEMPERED. STO HALL. CONICAL, FLANGED ENOS

DEG

90

90
90
90

IN IN.
2
2
3
6

CODE NO.

$72-8320$

$72-8340$

72-6040

$72-1950$

FLANGE, CAST IRON, AOAPTEO ASHE 125 LB FLANGE, REAMEO TO ACCEPT STO INSERTS. ND. 6B8-002, FISCHER ANO PORTER

SILE IN.

I

FLANGE, CAST IRON, LESS INSERTS, BOLTS AND NUTS, STYLE 2. FOR SID WALL CONICAL FLANGED ENDS. GOROSILICATE GLASS PIPE

$\begin{array}{ll}\text { PIPE IO IN. } & \text { CODE NO } \\ 1 & 72-9652 \\ 1112 & 72-9653 \\ 2 & 72-9654 \\ 3 & 72-9655\end{array}$

FLANGE, CAST IRON, SET INCLUDES 2 FLANGES, 2 INSERTS, W NUTS AND BOLTS, STYLE 2. 4 IN. ID PIPE. FOR STD NUTS AND BOLTS, STYLE 2. 4 IN IIDPIPE, FOR STD WALL CONICAL FLANGE

FLANGE, GLASS TO GLASS, ALUMINUM, BOROSILICATE GLASS, WITH INSERT, BOLT AND NUTS, FOR GLASS TUBING WITH INSERT, BOLT AND NUTS, FOR GLASS TUBING,
STYLE 1 . 4 IN. 10,6 . BOLT HOLES, CODE NO. 72-0538

FLANGE, CAST IRON, SET INCLUDES 2 FLANGES, 2 INSERTS. B NUTS AND BOLTS. STYLE 2,6 .IN. ID PIPE, BOROSILICATE GLASS PIPE. CODE NO. 72-0739

FLANGE, CAST IRON. FOR BOROSILICATE GLASS PIPE IO SILE IN.

$3 / 8$

$3 / 4$

FLANGE, PIPE, TYPE 316 SS, BLINO, DRILLED HIA.FS. MACHINED EDGES, IN. BOLT HOLE DIA, FOR STO WALL AND CCNICAL FLANGED ENDS BOROSILICATE. GLASS PIPE

$\begin{array}{lll} & \\ \text { BOL I CIRCLE } & \text { FDR PIPE } & \text { FLANGE } \\ \text { OIA IN. } & \text { SILE IN. } & \text { OD IN. } \\ 23 / 4 & 111 / 2 & 33 / 4 \\ 313 & 11 / 2 & 43 / 8\end{array}$

FLANGE, PIPE, TYPE 316 SS, BL IND, ORILLEO MOLES, MACHINED EDGES, $1 / 4$ IN. THK, FOR STD HALL AND CONICAL FLANGE ENDS BaROSILICATE GLASS PIPE BOLT CIRCLE BOLT BOLT HCLE FLANGE FOR PIPE DIA IN. $\begin{array}{llllll}\text { DIA IN. } & \text { HOLES } & \text { DIA IN. } & \text { OD IN. SIZE IN. } \\ 37 / 8 & 3 & 3 / 8 & 47 / 8 & 2 \\ 51 / 4 & 6 & 3 / 8 & 61 / 4 & 3\end{array}$

GASKFT. RIIIF ASRFSTRS FIAFR. FRB ARROSIIICATE GLASS PIPE. STYLE 1 PIPE SIZE IN. CODE NO. $\begin{array}{ll}11 / 2 & 72-9129 \\ 2 & 72-9130\end{array}$

UNI

EA

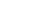$$
2
$$$$
15-321-1201 \quad 97
$$$$
\text { EA }
$$$$
\begin{aligned}
& 3.80 \\
& 5.86
\end{aligned}
$$

3

$15-321-4201$
$15-321-4221$

97
97

EA
EA

EA

$\begin{array}{llll}4.84 & 3 & 15-321-4401 & 97 \\ 6.37 & 3 & 15-321-4411 & 97 \\ 8.93 & 3 & 15-321-4421 & 97\end{array}$

3.57

3

$15-322-102$

97

EA
EA
EA

9.11
13.39

20.86
65.25

23
2
2
2

15-322-2271

$15-322-2291$

$\begin{array}{ll}15-322-2301 & 97 \\ 15-322-2321 & 97\end{array}$

$\begin{array}{lllll}\text { EA } & 2.48 & 2 & 15-322-5100 & 97 \\ \text { EA } & 4.76 & 2 & 15-322-5120 & 97\end{array}$

EA $\quad 1.81$

EA $\quad 1.50$

$\begin{array}{ll}E A & 1.99 \\ E A & 3.34\end{array}$

23
23
23
23

15-322-6101

$15-322-6121$

$\begin{array}{llll}1.34 & 23 & 15-322-6141 & 97\end{array}$

SET

20.72

23

$15-322-618$

SET

16.64.

$15-322-634$

97

SET

39.31

$15-322-6421$

97

$\begin{array}{lllll}\text { EA } & .70 & 3 & 15-322-6501 & 97 \\ \text { EA } & .70 & 3 & 15-322-6511 & 97 \\ \text { EA } & .57 & 3 & 15-322-6516 & 97\end{array}$

\section{EA}

12.50
22.50

2.50
2.50

$15-322-6821$

97
97

$\begin{array}{lrrrr}\text { EA } & 22.17 & 2 & 15-322-6861 & 97 \\ \text { EA } & 6.45 & 2 & 15-322-6881 & 97 \\ & & & & \\ & & & & \\ \text { EA } & .17 & 3 & 15-324-1007 & 97 \\ \text { EA } & .55 & 3 & 15-324-1009 & 97 \\ \text { EA } & .40 & 3 & 15-324-1011 & 97\end{array}$


IUBE_FIIUNGS. EOB BOROSWLICAIE GLASS_RIPE

GASKET, CHEMISEAL, TEFLON ENVELOPE WITH 1/16 IN. NEOPRENE FILLER, FOR USE WITH CORNING TYPE CONICAL FLANGE ON PYREX GLASS PIPE

PIPE SILE NO. BDLT CIRCLE - CORNING

IN. OD HOLES DIA IN.

$\begin{array}{llll}1 & 3 & 23 / 4 & \text { CODE NO. } 72-9138 \\ 11 / 2 & 3 & 31 / 2 & \text { CODE NO. }\end{array}$

GASKET, INTERFACE, 3/8 IN.., FISCHER AND PORTER CO, FOR PYREX PIPE

GASKET, FLANGE, BOROSILICATE GLASS, INTERFACE, TRIANGULAR SHAPE, I/2 ID IN., FISCHER AND PORTER

GASKET, PIPE ADAPTOR, KOROSEAL, FOR BOROSILICATE

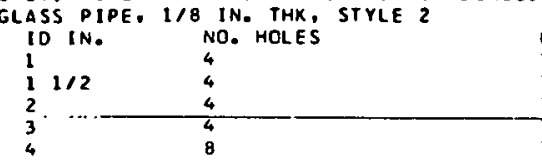

CODE NO.

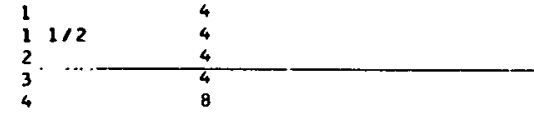

$72-9163$
$72-9164$
$72-916$
$72-916$

4
5
6

PRICE STK PT CATALOG NO GUYER

GASKET, PIPE, STO, KOROSEAL, FOR BOROSILICATE GLASS

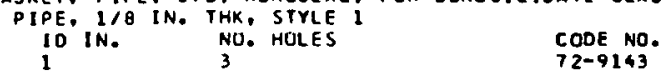

GASKET, TEFLON, TYPE T, WITH SELF-CENTERING CLIPS

AND RIDGES. MAX MORKING TEMP 45 DEG $F$. FOR

USE WITH CONICAL ENO GOROSILICATE GLASS PIPE.

WILL PROCESS EQUIPMENT CO, STYLE 1

$\begin{array}{ll}\text { SILE IN. CODE NO. } \\ 1 \\ 11 / 2 & 72-9254 \\ 2 & 72-9255 \\ 3 & 72-9256 \\ 4 & 72-9257 \\ 6 & 72-9808 \\ 72-9809\end{array}$

INSERT, Flange, asbestos, molded, Res In BONDED, ONE

PC CONSTRUCTION, FOR STO WALL BOROSILICATE GLASS

TUBING IPIPEI. CORNING

\begin{tabular}{|c|c|c|}
\hline $\begin{array}{l}10 \text { IN. } \\
11 / 2 \\
21 / 2\end{array}$ & $\begin{array}{l}\text { CODE NO. } \\
72-9056 \\
72.7057\end{array}$ & $\begin{array}{l}\text { NUMBER } \\
729056 \\
720057\end{array}$ \\
\hline & $72-9058$ & 729058 \\
\hline
\end{tabular}

INSERT, FLANGE, ASBESTOS, 2-PC, FOR SID WALL 8ORO-

SILICATE GLASS TUBING. NO. P-22, CORNING

IU IN. CLLAS COBE NB.

4 IN. COBE 118

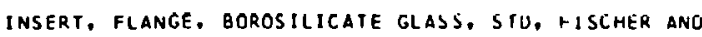
POR TER 10 IN.

$$
1 / 2
$$

INSERT, FLANGE, TEFLON, FOR SIO HALL BORCSILICATE GLASS PIPE

IO IN.

3

JOINT, SPHERICAL. STAINLESS STEEL, S/T JCINT 16/9,

THREAD 1/4 IN.. 0.540 IN. OD, NO. 7658. ACE

FEMALE

MAL C

REDUCER, BOROSILICATE GLASS, PIPE SIZE $11 / 2 \times 1 / 2$

IN.. NO. 670-IOQ, FISCHER AND PORTER

REOUCER, GOROSILICATE GLASS PIPE, TEMPERED, STO HALL,

CONICAL FLANGED ENDS, PYREX 7740 OR KIMAX KG-33
IO IN.
LGTH IN. CODE NO.

$\begin{array}{lll}\text { ID } I N . & \text { LGTH IN. } & \text { CODE NO. } \\ 1 \times 1 / 2 \times 1 & 4 & 72-0050 \\ 2 \times 1 & 4 & 72-0060 \\ 3 \times 1 & 5 & 72-0070 \\ 3 \times 1-1 / 2 & 5 & 72-0090 \\ 3 \times 2 & 5 & 72-0100 \\ 4 \times 1 & 7 & 72-0760 \\ 4 \times 3 & 7 & 72-0360 \\ 6 \times 1 & 9 & 72-2170\end{array}$

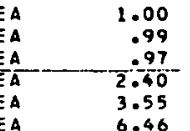

2
2
2
2
2
2

$15-324-2102$

$15-324-2104$

$15-324-2106$

$15-324-2106$
$15-324-2108$

$\begin{array}{ll}15-324-2110 & 97\end{array}$

BOROSILICATE GLASS, 90 DEG, $1 / 2$ IN, ID, FISCMER AND PORTER

\begin{tabular}{|c|c|c|c|c|}
\hline $\begin{array}{l}\text { EA } \\
\text { E A }\end{array}$ & $\begin{array}{l}.50 \\
.7 \mathrm{~A}\end{array}$ & $\begin{array}{l}23 \\
23\end{array}$ & $\begin{array}{l}15-324-3102 \\
15=326-3112\end{array}$ & $\begin{array}{l}97 \\
97\end{array}$ \\
\hline $\begin{array}{l}E A \\
E A\end{array}$ & $\begin{array}{r}.91 \\
1.12\end{array}$ & $\begin{array}{l}23 \\
23\end{array}$ & $\begin{array}{l}15-324-3122 \\
15-324-3132\end{array}$ & $\begin{array}{l}97 \\
97\end{array}$ \\
\hline $\begin{array}{l}E A \\
E A\end{array}$ & $\begin{array}{l}1.33 \\
1.45\end{array}$ & ${ }^{2} 3$ & $\begin{array}{l}15-324-3142 \\
15-324-3152\end{array}$ & $\begin{array}{l}97 \\
97\end{array}$ \\
\hline $\begin{array}{l}\text { EA } \\
\text { EA }\end{array}$ & $\begin{array}{l}.13 \\
.13\end{array}$ & $\begin{array}{l}3 \\
3\end{array}$ & $\begin{array}{l}15-324-3312 \\
15-324-3322\end{array}$ & $\begin{array}{l}97 \\
97\end{array}$ \\
\hline $\begin{array}{l}\text { EA } \\
\text { EA }\end{array}$ & $\begin{array}{l}8.00 \\
9.95\end{array}$ & 2 & $\begin{array}{l}15-324-3522 \\
15-324-3532\end{array}$ & $\begin{array}{l}97 \\
97\end{array}$ \\
\hline $\begin{array}{l}E A \\
F \Delta\end{array}$ & $\begin{array}{l}14.42 \\
17.73\end{array}$ & $\begin{array}{l}3 \\
3\end{array}$ & $\begin{array}{l}15-324-4420 \\
15-374-4425\end{array}$ & $\begin{array}{l}36 \\
36\end{array}$ \\
\hline$E A$ & 14.10 & 3 & $13-3 \angle 4-500<$ & 4 \\
\hline $\begin{array}{l}E A \\
E A \\
E A \\
E A \\
E A \\
E A \\
E A \\
E A\end{array}$ & $\begin{array}{r}8.01 \\
10.73 \\
16.35 \\
16.35 \\
23.10 \\
23.73 \\
23.73 \\
34.91\end{array}$ & $\begin{array}{l}2^{3} \\
3 \\
3 \\
3 \\
3 \\
2^{3}\end{array}$ & $\begin{array}{l}15-324-5202 \\
15-324-5212 \\
15-324-5232 \\
15-324-5242 \\
15-324-5252 \\
15-324-5262 \\
15-324-5292 \\
15-324-5302\end{array}$ & $\begin{array}{l}97 \\
97 \\
97 \\
97 \\
97 \\
97 \\
97 \\
97\end{array}$ \\
\hline Ex & 3.32 & 9 & $15-324-711$ & \\
\hline
\end{tabular}


IURE_ELIIIHGS. FOB BQROSWWGAIE GLASS_PIPE

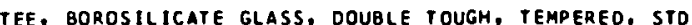
WALL. CONICAL FLANGEO ENOS. CORNING

ID IN.
1

$272-8250$

TEE, REDUCING, 8OROSILICATE GLASS PIPE, TEMPERED, STO WALL. CONICAL FLANGEO ENDS. PYREX 7740 OR KIMAX

IO IN. CODE NO.

IUBEELIINGS_ERASIC.

JOINT. BALL, POLYETHYLENE, SIZE 12/3, NO. 1322. LAB-LINE

JOINT, SOCKET, POLYETHYLENE, SIZE 12/3, NO. 1324, LAB-L INE

_IUBE_EIIIWGS._EOB_OUABIL_PIPE.

JOINT, GROUMO, BALL. AND SOCKET, BALL ONLY, FUSEO, TRANSPARENT, UCC SPEC Y-MS-121

SI2E

$28 / 15$

JOINT. GROUND, BALL AND SOCKET, SOCKET ONLY, FUSEO,

TRANSPARENT, UCC SPEC Y-MS-121

$5 I Z E$

$28 / 15$

JOINT, GROUND, STO TAPER, GLEAR FUSED OHARTZ, GE

INNER PART

Or NO

12130
14135

$40 / 50$

55150

60150

$71 / 60$

DUTER PART

OT NO.

or no.

12130

14135

$\frac{40150}{45750}$

55150

$60 / 50$

JOINT. GROUND. STO TAPER, FULL LGTH, FUSED, TRANS-

PARENT, UCC SPEC Y-MS-121

TYPE

FEMALE

FEMALE $\quad 24 / 40$

FEMALE $\quad 29 / 42$

FEMALE

FEMAL

MALE

MALE

MALE

50750

$19 / 38$
$24 / 40$

$34 / 65$

$50 / 50$

JOINT, SEMI-BALL. FUSED, TRANSPARENT, SEMI-BALL NO.

12/5. BALL DIA 12 MH, TUBE ID 5 MK. LGTH 6 IN..

AMERSIL CO, UCC SPEC Y-MS-121

TYPE

INNER BALL

OUTER SOCKET

JOINT. STO GALL AND SOCKET. CLEAR FUSEO QUARTZ, GE

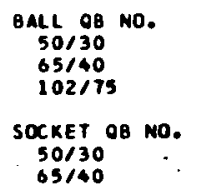

Attachment B

UNIT PRICE STK PT CATALOG NO BUYER

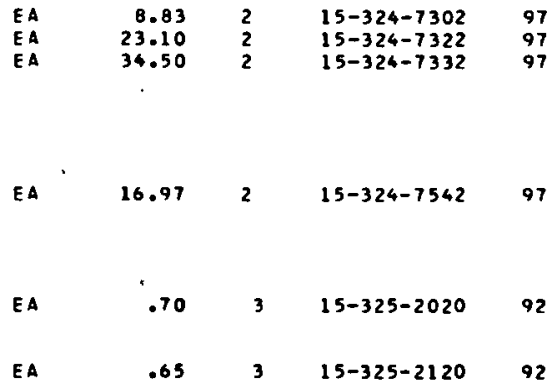

$\begin{array}{llrll}\text { EA } & 3.03 & 23 & 15-327-2100 & 97 \\ \text { EA } & 4.36 & 3 & 15-327-2120 & 97 \\ \text { EA } & 6.91 & 23 & 15-327-2140 & 97\end{array}$

$\begin{array}{lllll}\text { EA } & 3.15 & 3 & 15-327-2600 & 97 \\ \text { EA } & 4.73 & 3 & 15-327-2620 & 97 \\ \text { EA } & 7.51 & 3 & 15-327-2640 & 97\end{array}$ 
Attachment B

_UBE_EIIIINGS_FOB_QUABIZ_RIRE

JOINT. STO BALL AND SOCKET, CLEAR FUSFO QUARTZ, GE SOCKET OB NO.

$$
102175
$$

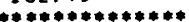

TUBULATION, BOROSILICATE GLASS, FOR HEAVY HALL FILTERING FLASK NO. 5341 . CORNING PYREX

UNOPETTE, RBC DILUTING SYSTEM, PARENTERAL SOLUTION FOR 200 TO 1 DILUTION. CONTAINER OF 100 TESTS 10 MICROLITER PIPETTE. B * D 5802

UNOPETTE, WBC OILUTING SYSTEM, DETERGENT SOLUTION FOR 100 TO 1 DILUTION. CONTAINER OF 100 TESTS MICRO IIIER PIPETTE AND 50 OILUENT VIALS, B D 5805

UNOPETTE PIPETTE, 100 PER PKG, B ANO D

CAP. LAMBDA

\section{NO.} 5888

VALVE, CHECK, GROUND FLOAT AND SEAT, HOLLOW BALL. PYREX GLASS, STYLE B, FOR VERTICAL USE, GROUND FLOAT MUST BE COMPLETELY ROUND WITH NO TAILS. NO. JV885O. SGA

VALVE. NEEDLE, TEFLON ANO GLASS, LESS GLASS CONN TUBES. EMIL GRENIER G10428, MANOSTAT 78-425-0 OR CMS 237-271

VALVE, ULTRAMAX, TEFLON, USEO CN LABORATORY GLASSWARE. SCIENTIFIC GLASS APPARATUS CO PART

KNOB, HEXAGONAL, NYLON

O-RING. COMPRESSION

PLUG, TURNING, TEFLON

WASHER, TEFLON
UNI T

PRICE STK PT CATALOG NO BUYER

- CONI I NUEO-

-CONTI NUFD-
VIAL. CELLULOSE ACETATE, 5 ML CAP.. 2 TO $21 / 2$ IN HIGH BY $1 / 2$ IN. DIA.. $125 \mathrm{MG} / \mathrm{CA}$ TO THE SECOND poner halli hith gasketed nickel plateo steel cap. E-52. TRACERLAB

masher, cage ano rack, automatic, steam heated, mODEl NO. 6000. BETTER BUILT MACHINERY CORP PART

PT NO.

ARM, SPRAY, STAINLESS STEEL, FOR HUB OF ROTARY HEADERS

CLR-IF

AXLE, STAINLESS STEEL, FOR HUB OF

CLA-37A ROTARY HEADERS

BUSHING, BEARING

COIL, I IN., SOLENOTD VAI VE

CYIIMDER, AIR, 2 IN.

DIVERTER: TIMER, MOJUSTABLE

FILTER, AIR, FOR BELL-GOSSETT AIR

COMPRESSOR, RODEL SYC-T-34-1AO

GLASS, VIEH-PORT

HUB. MACHINED CASTING, COMPLETE HITH

INSERT PRESS FIT BEARING AND TWO

STAINLESS STEET, SET SCREHS. FOR ALL

ROTARY HEADERS

LOCK, BOOY ASSY

LUBRICATOR-FILTER, AIR LINE, 1/8 IN.. NO. FL-125, BELLOHS CO

PLATE, CAPPING, STAINLESS STEEL, FOR MUB OF ROTARY HEAD

CLR-1C

PLUG, SPRAY aRMS, ROTARY HEAOERS

CLR-1G

REGULATOR, TEMPERATURE, 3/4 IN.

CLB-13

CLR-IM

CLR-1E

CLB-4h

CLB-98

$\rightarrow$

CLA-5

$-$

COMPLETE

REGULATOR, TEMPERATURE, COMPLETE, FOR

USE MITH 100 GAL GENERATOR, NO. A1413T -

9R2. ASMCRDFT, 2100 INOICATING TYPE,

2200 MOM-INDICATINC TYPE, RANGE - GOLO

SPR ING 130 TO 190 OEG $F$. OR 55 TO 85 DEG

C. RANGE - CAOHIUH SPRING 130 TO 220 DEG

F. OR 55 TO 100 DEG C. 1112 IN.
CONT

$P K$ PKG

EA

EA

EA
EA
EA

A

$64.00 \quad 3 \quad 15-328-1170 \quad 97$

A $.29 \quad 3 \quad 15-331-1200 \quad 36$

CNT $6.7525^{\prime} 15-333-1500$ ' 36

$\begin{array}{lll}0.29 \quad 2 & 15-333-1520 \quad 36\end{array}$

.

$\begin{array}{rlll}13.07 & 2 & 15-333-1600 & 36 \\ 8.50 & 2 & 15-333-1620 & 36\end{array}$

$6.78 \quad 3 \quad 15-336-4500 \quad 36$

$6.66 \quad 3 \quad 25-336-6000 \quad 36$

$\begin{array}{llll}.30 & 3 & 15-396-6210 & 36 \\ .24 & 3 & 15-336-6230 & 36 \\ 1.00 & 3 & 15-336-6240 & 36 \\ .25 & 3 & 15-336-6250 & 36\end{array}$

E A

.40

$15-336-6800 \quad 36$

EA

$72.502 \quad 25-337-1500 \quad 35$

E A

15.00

4.50

8.50
95.00

95.00
35.00

2 15-337-1505

15-337-1515

$13-331-1525$

15-337-1540 35

EA

3.50

15-337-1550 35

EA

14.50

15-337-1555 35

EA $90.00 \quad 2 \quad 15-337-1560$ is

EA $48.76 \quad 2 \quad 15-337-1565 \quad 35$

$202-099-5180$.

EA $\quad 6.25 \quad 2 \quad 15-337-1580 \quad 35$

EA $\quad .60 \quad 2 \quad 15-337-1585 \quad 35$

EA $115.00 \quad 2 \quad 15-337-1590 \quad 35$

EA $140.00 \quad 2 \quad 15-337-1595 \quad 35$ 
Att achment B

UNIT PRICE STK PT CATALOG NO BUYER

HASHER, RACK, STEAM STERILILER, MOOEL 21-63-170, TYPE OC, TYPE II. R G WR IGHT CO, INC PAR T

PT NO.

SWITCH, TOGgle, SINGle PHASE, 2 POLE, OPEN TYPE, NO. GOOTOX5, ALLEN-BRAOLEY --

SWITCH, TOGGLE, NO. 0111-0002, MCGILL

TRANSFORMER, 300 KVA, TYPE SZO, NO. 48319, HEVI-DUTY ELECTRIC CO --

VALVE, tRANSFER, SHIVEL. NICKEL PLATE 21-8397

MATCH GLASS, ANNEALEO, MEDIUM THICKNESS, CONCAVE, FINISHED EOGES, NO. 28141 . WILL CORP

OIA MM

40 (MATKESON $04200-10$ )

50 IMATHESON $64200-15$

65 (MATHESON $64200-20$ )

75 IMATHESON $64200-25$

90
100 (MATHESON $64200-40$
(MATHESON $64200-35$ )

115 IMATHESON 64200-40

125 (MATHESON $64200-45$ )

140 TRATRESON $64200-50$

175 (MATHESON $64200-65$ )

250 FISHER 2-610, SARGENT $5-83605-X$

WATCH GLASS, COUNTERPOISED, $75 \mathrm{MM}$ OIA, WILL CORO NO. 28161. MATHESON 3040-30. PREISER 10-1.760/20

MaTCH GLASS, PLAIN. SOFT GLASS, FullY ANNEALEO. CONCAVE SHAPE. O.984 IN. 0.005 IA. TO 0.010 IN. CONCAVE SHAPE. O.984 IN. O.0O5 IA. TO O.010 IN, DIA. O.04T IN. THK

WATCH GLASS, PYREX GLASS, HEAYY WALL, UNIFORM FIRE POL IŚHED EOGE, NO. 9985. CORNING

OL IS HE O

05

75

90
100

WATCH GLASS, SPEEOYVAP WITH.THREE RIBS MCULDED INTO

UNDER SIOE FOR LOW FORM BEAKER, NO. $\mathrm{H}-64220$,

HAR SHAM

\begin{tabular}{|c|c|}
\hline $\begin{array}{l}\text { DIA IN. } \\
3 \\
31 / 2\end{array}$ & $\begin{array}{l}\text { (VUR } 66114-022) \\
\text { MATHF SON } \\
\text { MA220-15 }\end{array}$ \\
\hline & (VHR 66114-066) \\
\hline 5112 & $\begin{array}{l}\text { MATHESON } 64220-25 \\
\text { MATHE SON } 64220-30 \\
\text { MATHESON } 64220-35\end{array}$ \\
\hline
\end{tabular}

MATCH GLASS, TRANSPARENT VITREOSIL, $31 / 2$ IN. DIA, THERMAL SYNDICATE LTO

WATCH GLASS, OUARTL, FUSED, TRANSPARENT, $41 / 2$ IN. OIA

maX. SEALING, APIEZON TYPE O, 2 LB CAN, JAMES $G$ B IOULE CO. NO. 14-638-20, FISHER

MAX, SEAL ING, APIEZON TYPE $W$, STK APPROX 7 IN. LGTH, JAMES G BIDOLE OR LAPINE 35376-29X

WAX, UNIVERSAL RED, L IOUIFIES AT APPROX 65 DEG $C$. 2 O2 STICK. CENCO NO. 11450 OR FSCO 25-530

WEIGHT SET, aNALYTICAL, NoB.S. Class S, I MG TO 100 GM. FACTORY TO FURNISH A COMPLIAMCE STATEMENT THAT MEIGHTS MEET REQUIREMENTS FOR N.B.S. CLASS S MEIGHTS. NO, 4254-S. M AINSMORTM AND SONS

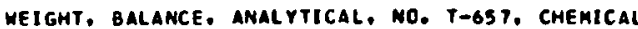
RUBBER CO

DENOMINATION MILLIGRAM

20 OHAUS 51021

$\begin{array}{ll}200 & \text { OHAUS } 51022 \\ 300 & \text { OHAUS } 51052\end{array}$

WEIGHT, BALANCE, METRIC, BRASS, I GM TO 1000 GM PER SET. IN OPEN BLOCK, FOR MARVARD TRIP BALAMCES. TORSION BALANCES, ETC, MO. 3420-60, MATHE SON

OR ND. 2-301. FISHER
- CONT I NUEO-

$\begin{array}{rrrrr} & & 2 & 05-108-1087 & \\ & & & & \\ & & & 15-337-8195 & 35 \\ & & 2 & 15-337-2576 & \\ & & 2 & & \\ & 230.95 & 2 & 15-337-8225 & 35\end{array}$

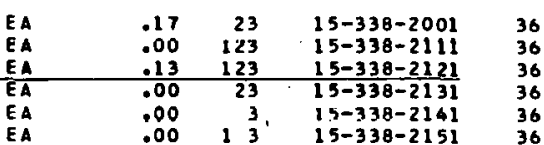

EA

CAN

STK

STK

SET

175.00

.00

15-338-3001

36

EA
EA A

.00
.00
.00

15-998-3061 $15-338-3091$
$15-338-3101$

30
36
36

SET 


\section{CRITERIA CHECKLIST \\ SCIENTIFIC GLASSBLOWING FACILITY}

SPACE

A gross floor area of about 500 square feet is a desirable planning allowance for a completely equipped glass shop for one glassblower. Two or more glassblowers, however, need only about 250 square feet per man. A floor plan with all equipment located is one of the best planning aids.

\section{MACHINERY}

1. Lathe, glassblowing (number and size determined by expected work - at least one for each two glassblowers)

2. Drill press, 1/2" pedestal, variable speed

3. Glass saw with pass-thru feature

4. Flat lap, rotating, variable speed

5. Belt grinder, vertical

\section{EQUIPMENT}

1. Oven, annealing, $1400^{\circ} \mathrm{F}$

2. Sink, stainless steel with drainboard

3. Spot welder

4. Hand forming tools (general)

5. Bench burner

6. Hand torches (each workbench and lathe)

7. Glass cutter (plate and sheet)

8. Circle cutter (plate and sheet)

9. High vacuum system with a connector in oven

10. Water fountain, hot-cold

11. Personal safety glasses, neodymium and cobalt tints

\section{FURN ITURE}

A. Each Station

1. Workbench, glassblowers, 10' formica top

2. Stool, draftsman, adjustable

3. Base cabinet, storage, with drawers and cupboard 
B. General

1. Cabinets, storage

2. Benches, flattop utility

3. Racks, glass stock storage with separators for sizes

\section{UT ILITIES}

A. At each 1 athe and workbench

1. Compressed air

2.. Natural or LP gas

3. Oxygen

4. Hydrogen

5. House vacuum

6. Electrical outlets

B.. General

1. Water

2. Distilled water

HEATING., ..VENT.ILAT.ION, AIR CONDITIONING

Adequate heat exhaust through ceiling hoods over each lathe, workbench, and oven is important. A fume hood.or exhaust is required for chemical work at the sink. The high air volume requirements need consideration in sizing proper heating and cooling equipment, and also in distributing flow well to prevent drafts. Heat from the work aids in heating the shop, yet adds considerable load for air conditioning. Work station spot-cooling air distribution can aid in an economic compromise for worker comfort.

\section{LIGHT ING}

Illumination should be well distributed with 100 footcandles intensity at work stations other than the glassblowing benches where about 75 . footcandles is adequate and will probably result from overhead hood shadow. 


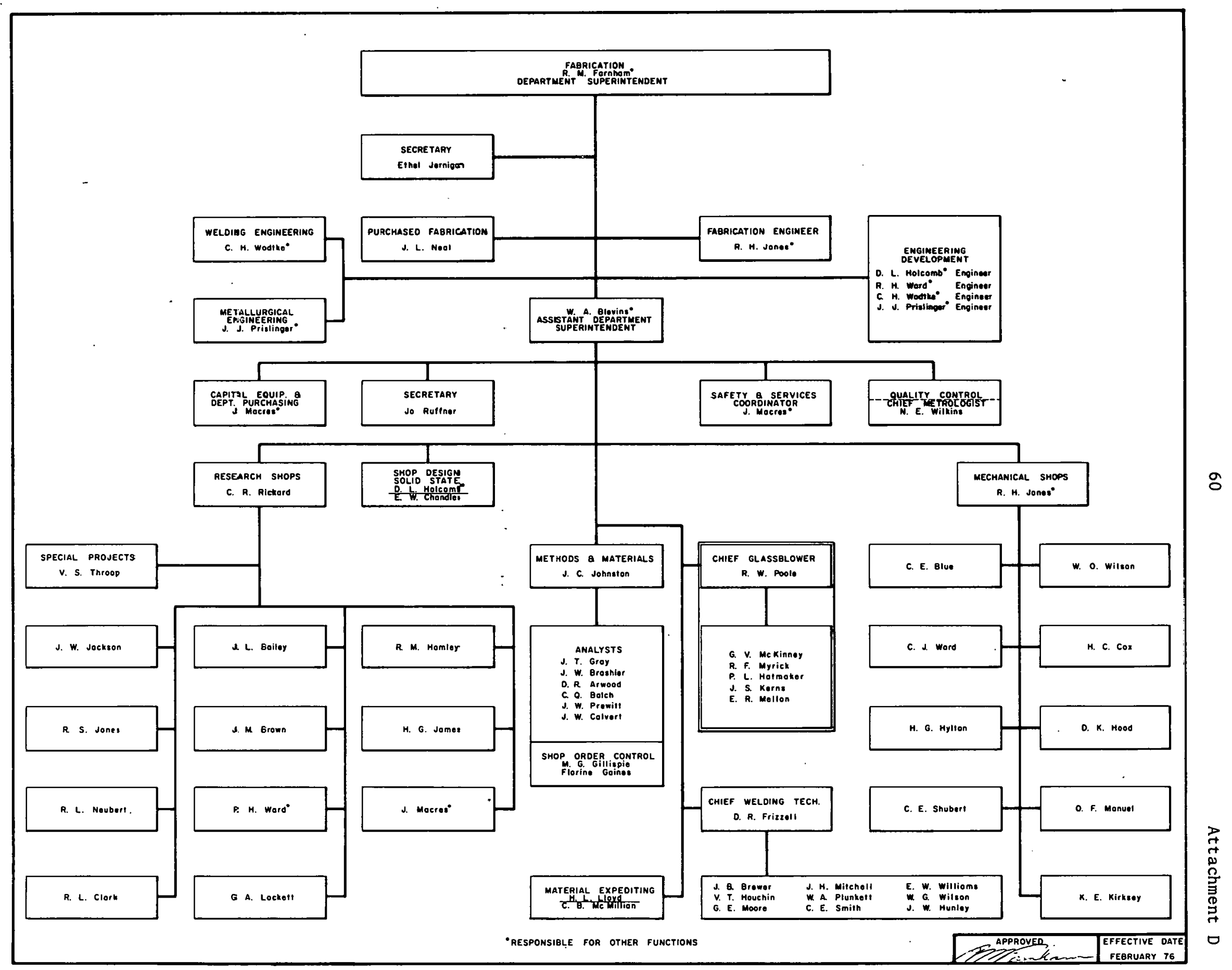


1. A. L. Allen

2. M. A. Baker

3. W. A. Blevins

4. D. Brogan

5. W. F. Buker

6. D. M. Davis

7. E. A. Davis

8. R. J.: DeBakker

9-29. R. M... Farnham

30 ... J.. W. Fields

31. C. L. Fox

32. D.. C. Gary

33. C.. R. Grubb

34. R., M.. Hartman

35.6 H. H. Haymond

36 ... P... W. Hembree

37. . W. S. Hornbaker

38.. R. . S. Jackson

39. K. E. Jamison

$40 \ldots$ R. G : Jenness

41.. R. H. Jones

42. H. F. Kèsee

43. J.: C. Lyel1

44... J.. Macres

45..... C.. E. Murphy .

46. H. H. Nichol

47. R., W. Poole

48. M. E. Ramsey

49.. C. R. Rickard

50-70.. . H. E. Seagren

71... C.. R. Sherlin

72... E. Taylor

73.. D.: C. Tuxbury

$74 \therefore$ R. . H. Winget

75.. G. . B. Young

76-77. Central Research Library

78. Document Reference Section

79-80. Laboratory Records Department

81.. Laboratory Records, ORNL (RC)

82. ORNL Patent Office

83. Research and Technical Support Division, ERDA-ORO

84-110. Technical Information Center, ERDA-ORO 